\title{
WestVirginiaUniversity
}

THE RESEARCH REPOSITORY @ WVU

Graduate Theses, Dissertations, and Problem Reports

2006

\section{A comparison of the relative efficiency of tracking signals in forecast control}

Balasubramanya Krishnamurthy

West Virginia University

Follow this and additional works at: https://researchrepository.wvu.edu/etd

\section{Recommended Citation}

Krishnamurthy, Balasubramanya, "A comparison of the relative efficiency of tracking signals in forecast control" (2006). Graduate Theses, Dissertations, and Problem Reports. 3261.

https://researchrepository.wvu.edu/etd/3261

This Thesis is protected by copyright and/or related rights. It has been brought to you by the The Research Repository @ WVU with permission from the rights-holder(s). You are free to use this Thesis in any way that is permitted by the copyright and related rights legislation that applies to your use. For other uses you must obtain permission from the rights-holder(s) directly, unless additional rights are indicated by a Creative Commons license in the record and/ or on the work itself. This Thesis has been accepted for inclusion in WVU Graduate Theses, Dissertations, and Problem Reports collection by an authorized administrator of The Research Repository @ WVU. For more information, please contact researchrepository@mail.wvu.edu. 


\title{
A COMPARISON OF THE RELATIVE EFFICIENCY OF TRACKING SIGNALS IN FORECAST CONTROL
}

\author{
Balasubramanya Krishnamurthy
}

Thesis submitted to

The College of Engineering and Mineral Resources

At West Virginia University

in partial fulfillment of the requirements

for the degree of

\author{
Master of Science \\ in \\ Industrial Engineering \\ Dr. Majid Jaraiedi, Chair \\ Dr. Wafik Iskander \\ Dr. Robert Creese
}

Department of Industrial and Management Systems Engineering

Morgantown, West Virginia

2006

Keywords: Tracking Signals, ARL1, Forecast Control, CUSUM, Smoothed Error, BCUSUM, PCUSUM, Autocorrelation 


\begin{abstract}
A Comparison of the Relative Efficiency of Tracking Signals in Forecast Control

\section{Balasubramanya Krishnamurthy}

An important aspect of forecasting system is monitoring the process for forecast accuracy. Tracking signals test is an effective detection method for occurrence of nonrandom changes. In this research, the relative efficiency of Cumulative Sum (CUSUM), Smoothed Error, Backward CUSUM, Autocorrelation and Parabolic CUSUM tracking signals were compared according to ARL1 criterion and percentage of trips detected within $\mathrm{N}$ periods. For simulating response to a step, 1,000 series of demands were generated, each covering run-ins of 40 periods and 75 periods after introduction of the step. The response of tracking signals to step sizes of $1 \sigma, 2 \sigma, 3 \sigma$ and a random step between $1 \sigma$ and $3 \sigma$ were studied at unbiased ARL of 25, 50 and 100. Smoothing parameters were varied from 0.05 to 0.2 in steps of 0.05 . When the smoothing parameter is set equal to $0.05,0.15$ or 0.2 and other smoothing constants are 0.05 , autocorrelation signal was the best choice. When the smoothing parameter is equal to 0.1 and other smoothing constants are 0.05 , BCUSUM was the best choice when unbiased ARL $=100$; autocorrelation signal was the best choice when unbiased $A R L=25$ or 50 . Autocorrelation signal is the best choice based on percentage of trips detected within $\mathrm{N}$ periods.
\end{abstract}




\section{Acknowledgements}

I would like to express my sincere gratitude to Dr. Majid Jaraiedi, for his continued support, guidance and encouragement during the course of this research work. I would also like to thank Dr. Wafik Iskander and Dr. Robert Creese, my committee members, for their advice and support. I am also very grateful to my parents, my sister and my fiancée, their help and encouragement were remarkable during my graduate studies. Last but not the least, I would like to thank my friends for their constant support and help in all my endeavors. 


\title{
Table of Contents
}

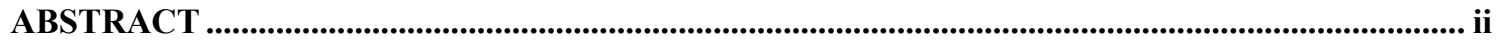

ACKNOWLEDGEMENTS ...................................................................................................................ii

TABLE OF CONTENTS .......................................................................................................................... iv

LIST OF FIGURES.................................................................................................................................. vi

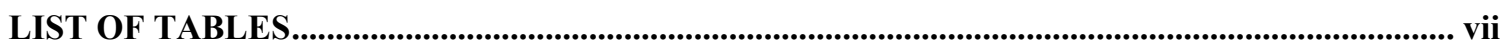

ABBREVIATIONS AND SYMBOLS.............................................................................................ii

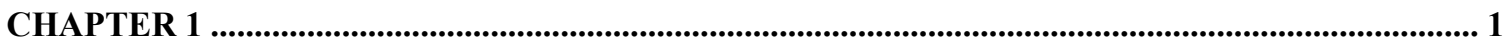

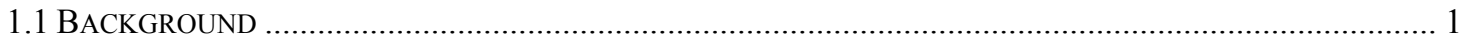

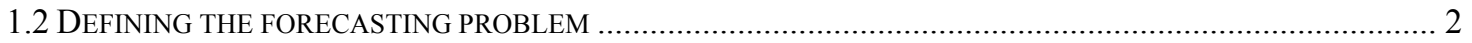

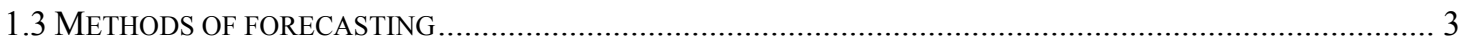

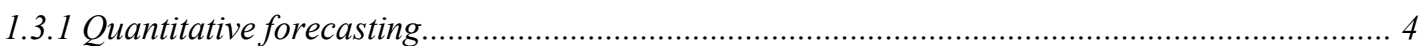

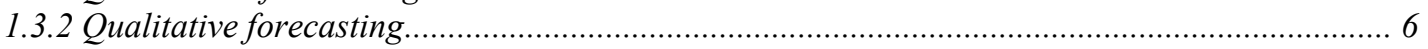

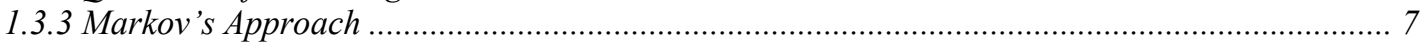

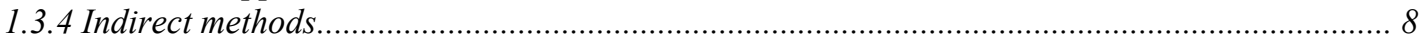

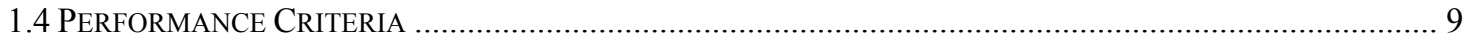

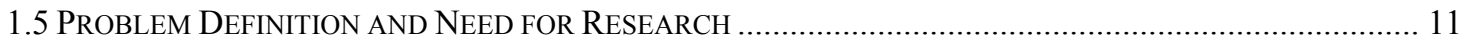

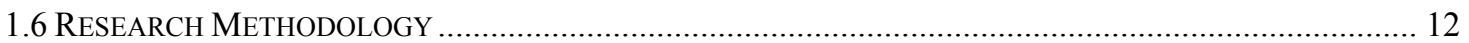

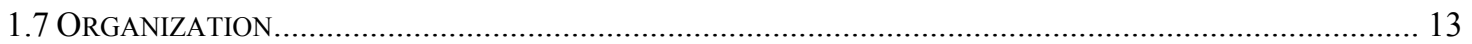

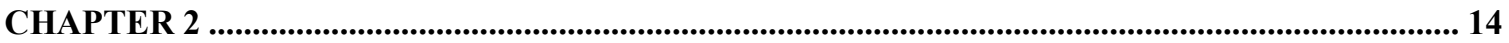

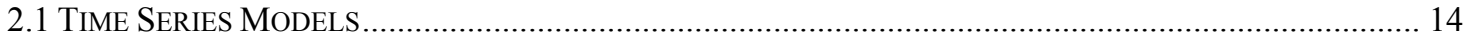

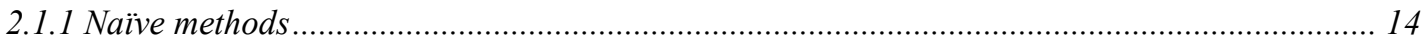

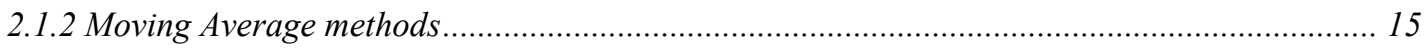

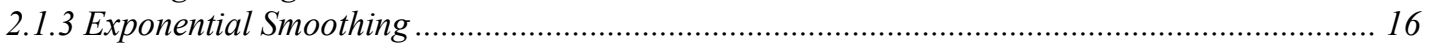

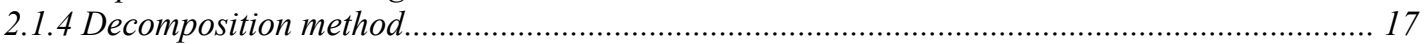

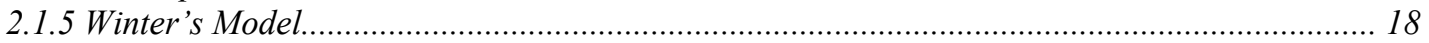

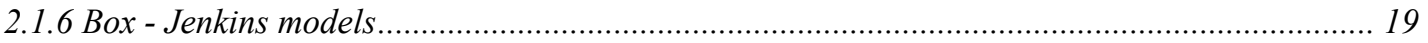

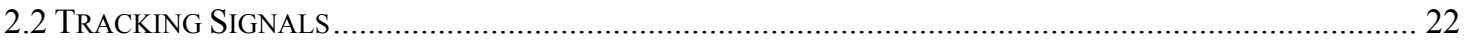

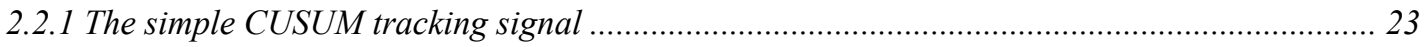

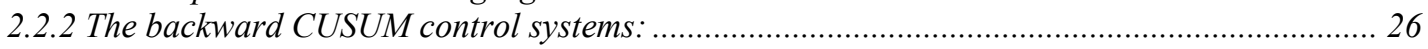

2.2.3 The smoothed error tracking signal:

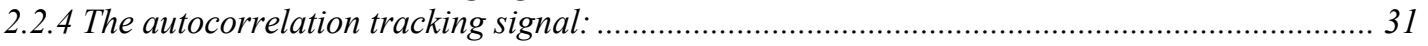

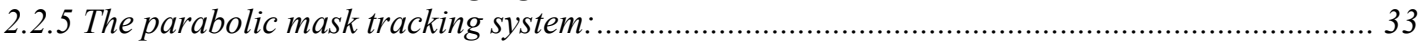

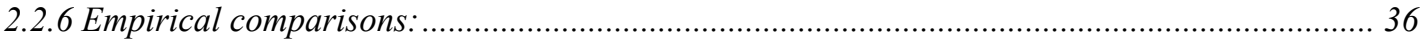

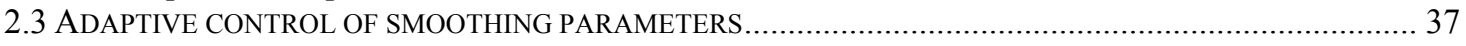

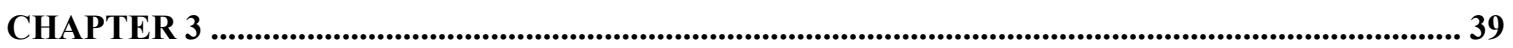

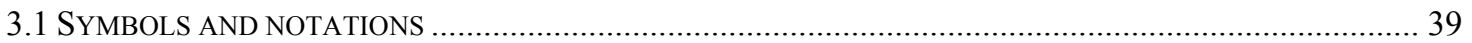

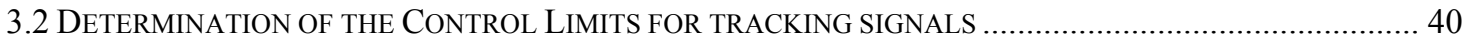

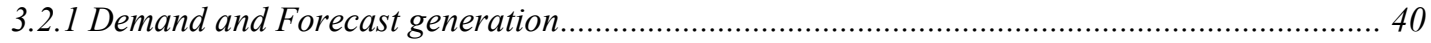

3.2.2 Control limits for CUSUM tracking signal .................................................................... 41

3.2.3 Control limits for Smoothed Error Tracking Signal (SETS) ................................................. 42

3.2.4 Control limits for Autocorrelation Tracking Signal (RTS).................................................... 42

3.2.5 Control limits for Backward CUSUM tracking signal (BCUSUM) ........................................ 45

3.2.5 Control limits for Parabolic CUSUM Tracking Signal (PCUSUM) ......................................... 49

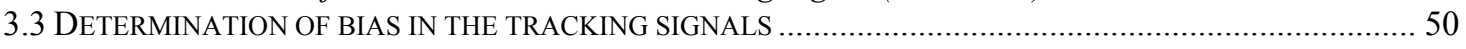

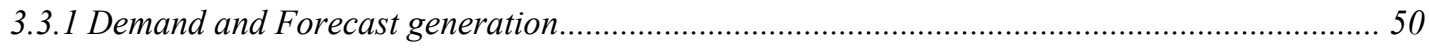




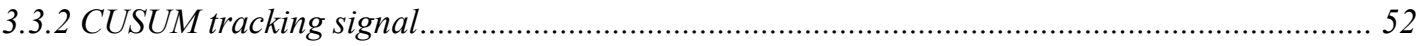

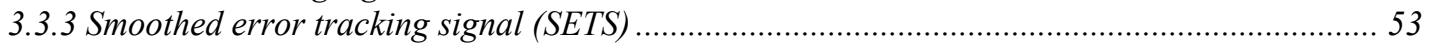

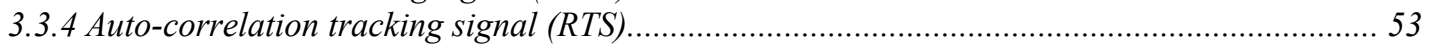

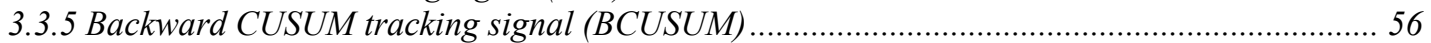

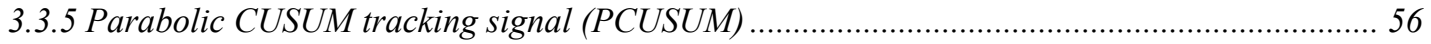

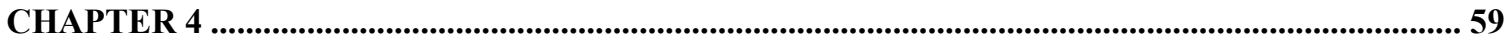

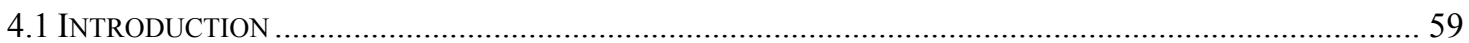

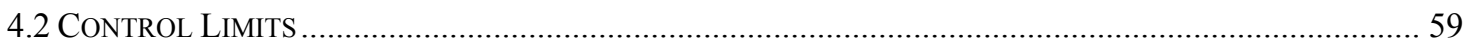

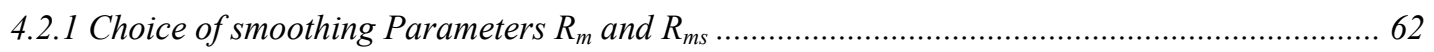

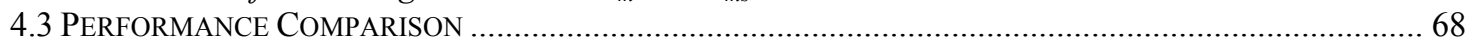

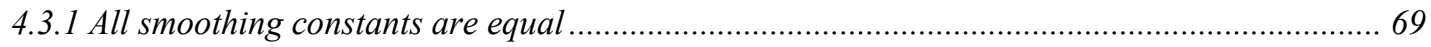

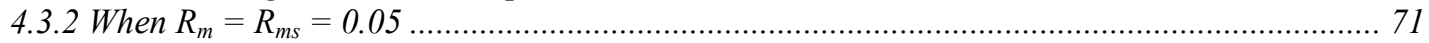

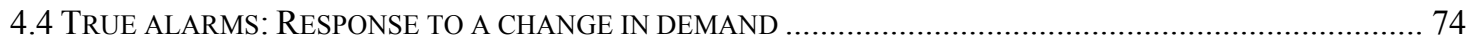

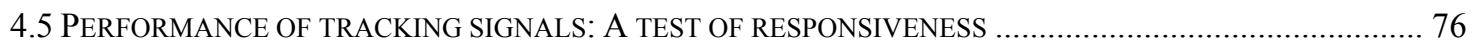

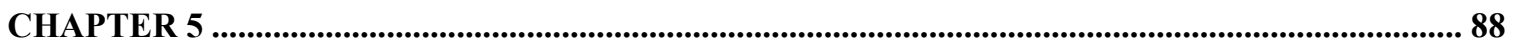

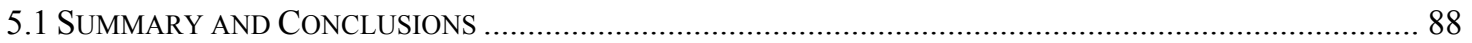

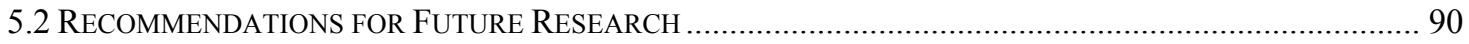

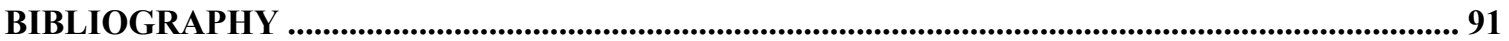




\section{List of Figures}

Figure 1.1 ForeCASTING MOdels (SHIM, Siegel, AND LiEW, 1994). 4

FigURE 1.2 THE FORECASTING SYSTEM (MONTGOMERY, JOHNSON AND GARDINER, 1990)

FigURE 3.1 PROCEDURE TO FIND CONTROL LIMITS FOR SIMPLE CUSUM $(A R L I=100)$

FIGURE 3.2 PROCEDURE TO FIND CONTROL LIMITS FOR SETS (ARL1=50) 44

FigURE 3.3 PROCEDURE TO FIND CONTROL LIMITS FOR RTS (ARL1=25) 46

FigURE 3.4 PROCEDURE TO FIND CONTROL LIMITS FOR BCUSUM $(A R L 1=50, W=0.1)$

FigURE 3.5 PROCEDURE TO FIND CONTROL LIMITS FOR PCUSUM $(A R L 1=50)$

FIGURE 3.6 PROCEDURE TO DETERMINE BIAS FOR CUSUM TRACKING SIGNAL.

FIGURE 3.7 PROCEDURE TO DETERMINE BIAS FOR SMOOTHED ERROR TRACKING SIGNAL. 54

FIGURE 3.8 PROCEDURE TO DETERMINE BIAS FOR AUTO-CORRELATION TRACKING SIGNAL.

FIGURE 3.9 PROCEDURE TO DETERMINE BIAS FOR BACKWARD CUSUM TRACKING SIGNAL. 57

FigURE 3.10 PROCEDURE TO DETERMINE BIAS FOR PARABOLIC CUSUM TRACKING SIGNAL. 58

Figure 4.1 FifTh PERIOd RESPONSE TO A $1 \sigma$ STEP (ARL=25) 77

FIGURE 4.2 RESPONSE WITHIN N PERIODS, ARL $=25, R_{A}=0.05,1 \sigma$ STEP

FIGURE 4.3 RESPONSE WITHIN N PERIODS, ARL $=25$, RA $=0.1,1 \sigma$ STEP 78

FIGURE 4.4 RESPONSE WITHIN N PERIODS, ARL $=25, \mathrm{R}_{\mathrm{A}}=0.15,1 \sigma$ STEP

FigURE 4.5 RESPONSE WITHIN N PERIODS, ARL $=25, \mathrm{R}_{\mathrm{A}}=0.2,1 \sigma$ STEP

Figure 4.6 FifTH PERIOD RESPONSE TO A $1 \sigma$ STEP $($ ARL $=50)$

FIGURE 4.7 RESPONSE WITHIN N PERIODS, ARL $=50, R_{A}=0.05,1 \sigma$ STEP

FIGURE 4.8 RESPONSE WITHIN N PERIODS, ARL $=50, R_{A}=0.1,1 \sigma$ STEP

FIGURE 4.9 RESPONSE WITHIN N PERIODS, ARL $=50, R_{A}=0.15,1 \sigma$ STEP

FIGURE 4.10 RESPONSE WITHIN N PERIODS, ARL $=50, R_{A}=0.2,1 \sigma$ STEP

Figure 4.11 FifTh PERIOd RESPONSE TO A $1 \sigma$ STEP $(A R L=100)$

FIGURE 4.12 RESPONSE WITHIN N PERIODS, ARL $=100, R_{\mathrm{A}}=0.05,1 \sigma$ STEP

FigURE 4.13 RESPONSE WITHIN N PERIODS, ARL $=100, R_{\mathrm{A}}=0.1,1 \sigma$ STEP

FIGURE 4.14 RESPONSE WITHIN N PERIODS, ARL $=100, R_{A}=0.15,1 \sigma$ STEP

FIGURE 4.15 RESPONSE WITHIN N PERIODS, ARL $=100, R_{A}=0.2,1 \sigma$ STEP $\quad 86$ 


\section{List of Tables}

TABLE 2.1 CuMULATIVE Distribution OF SIMPLE CUSUM TRACKING SIGNAL (GARDNER, 1983).............. 25

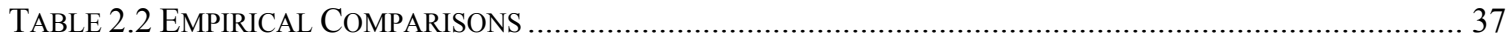

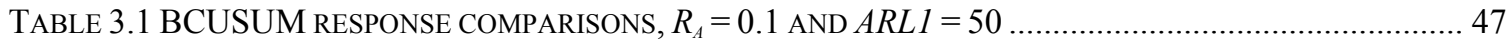

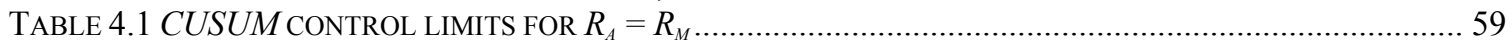

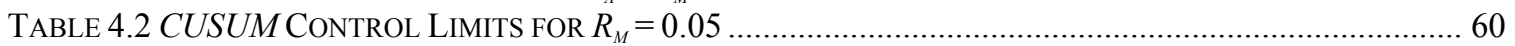

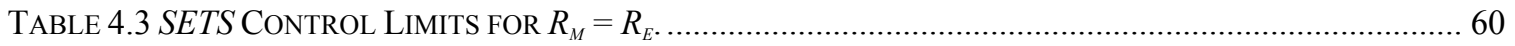

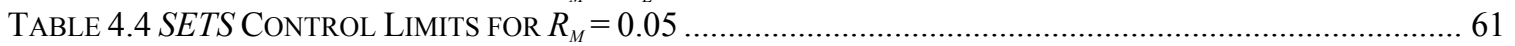

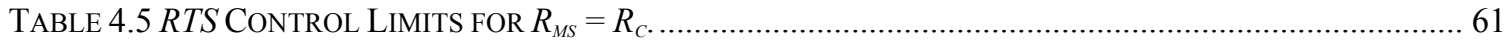

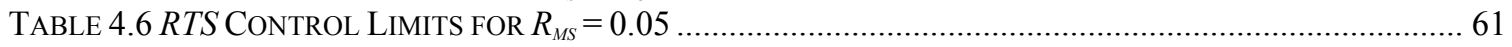

TABLE 4.7 ARL1 COMPARISON FOR DIFFERENT SMOOTHING PARAMETERS $R_{A}$ AT $1.5 \Sigma(S E T S) \ldots \ldots \ldots \ldots \ldots \ldots \ldots . . . . . . . . . .63$

TABLE 4.8 ARL1 COMPARISON FOR DIFFERENT SMOOTHING PARAMETERS $R_{A}$ AT $3 \Sigma(S E T S)$......................... 64

TABLE 4.9 ARL1 COMPARISON FOR DIFFERENT SMOOTHING PARAMETERS $R_{A}$ AT 1.5, $3 \Sigma$ (CUSUM) ............. 64

TABLE 4.10 ARL 1 COMPARISON FOR DIFFERENT SMOOTHING PARAMETERS $R_{A}$ AT $1.5 \Sigma(R T S)$...................... 65

TABLE 4.11 ARL1 COMPARISON FOR DIFFERENT SMOOTHING PARAMETERS $R_{A}$ AT $3 \Sigma(R T S) \ldots \ldots . . . . . . . . . . . . . . . . . .66$

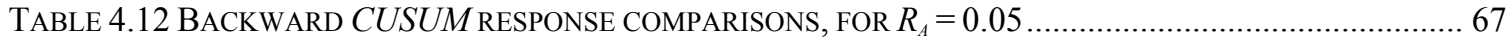

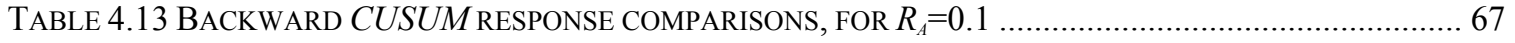

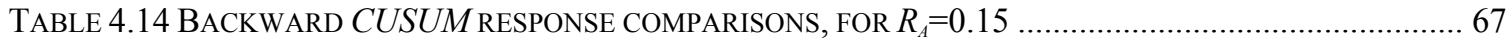

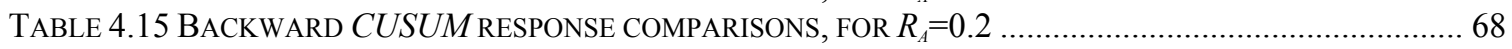

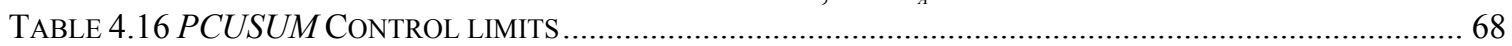

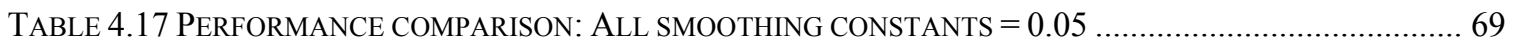

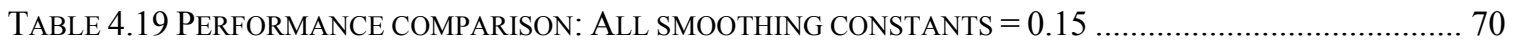

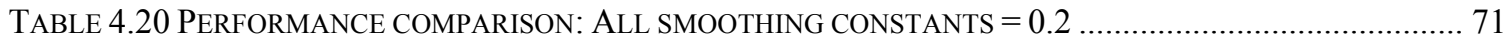

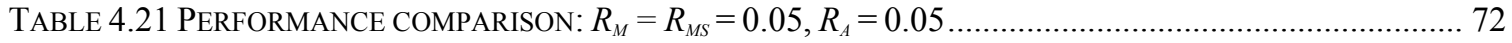

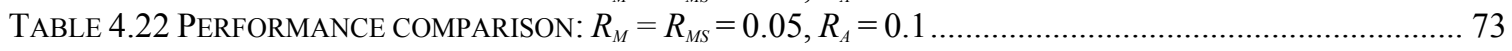

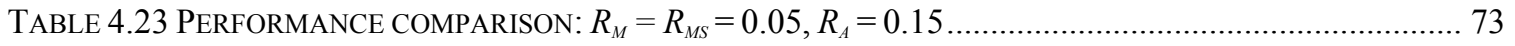

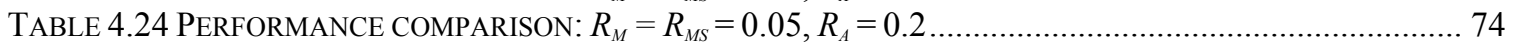

TABLE 4.25 RESPONSE TO A 1 SIGMA STEP $R_{M}=R_{M S}=0.05, R_{A}=0.1$, UNBIASED ARL $=50 \ldots \ldots \ldots \ldots \ldots \ldots \ldots \ldots \ldots . . . . . . . . . . .75$ 


\section{Abbreviations and Symbols}

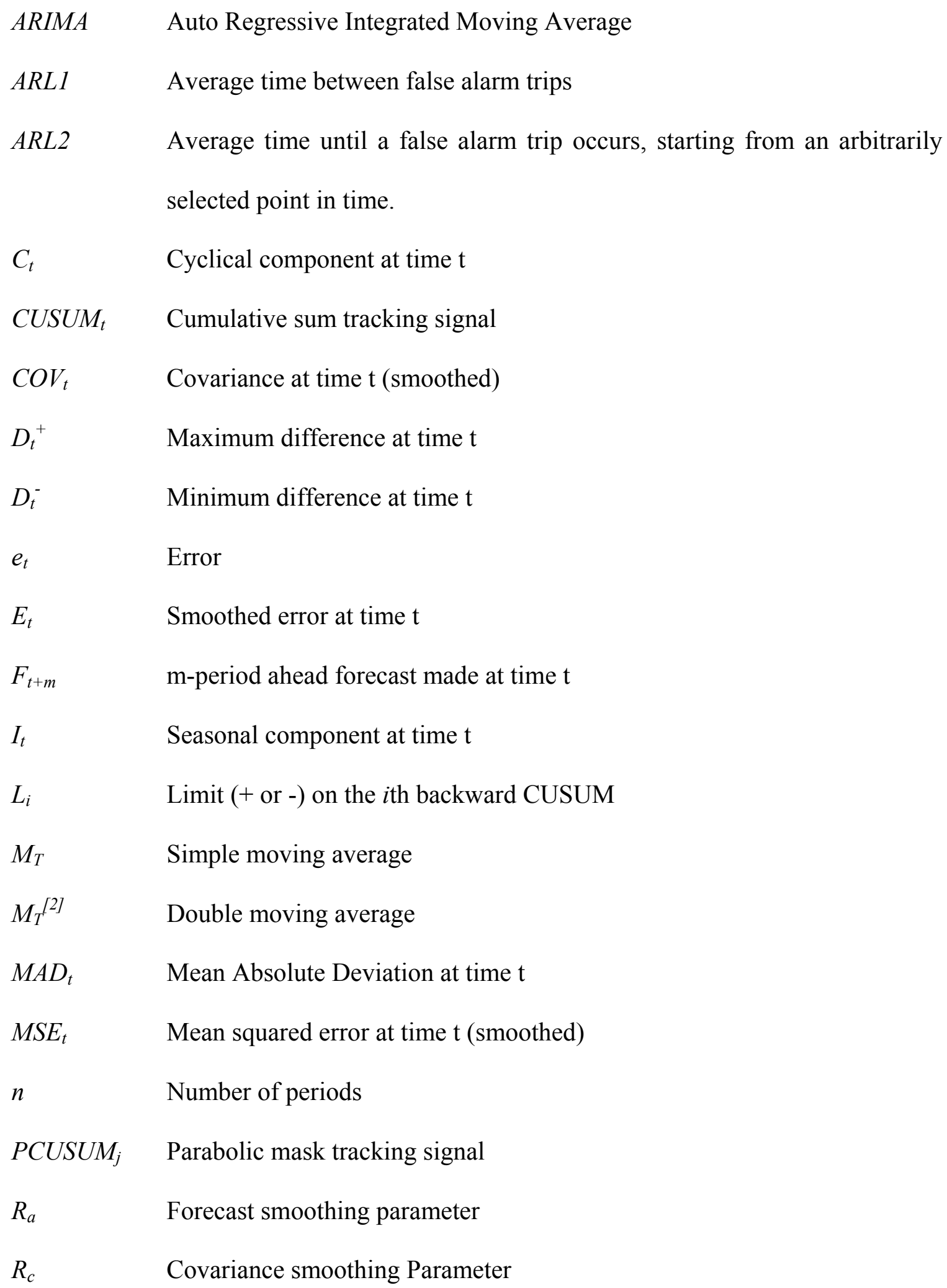




\begin{tabular}{|c|c|}
\hline$R_{e}$ & Error smoothing parameter \\
\hline$R_{m}$ & MAD smoothing parameter \\
\hline$R_{m s}$ & MSE smoothing Parameter \\
\hline$R T S_{t}$ & Autocorrelation tracking signal \\
\hline$S_{t}, S_{T}$ & Smoothed statistic at the end of time $t$ (Forecast for next period) \\
\hline$S_{T}^{[2]}$ & Double exponential smoothing statistic \\
\hline SETS $_{t}$ & Smoothed Error tracking signal \\
\hline SUMn & Sum of errors of $n$ periods \\
\hline$S U M_{t}$ & Sum of all previous errors at time $t$ \\
\hline$T_{t}$ & Trend component at time $t$ \\
\hline$\nabla^{d}$ & Backward difference operator of order ' $d$ ' \\
\hline$\alpha$ & Smoothing constant \\
\hline$\beta$ & Discount factor \\
\hline$\gamma$ & Trend smoothing co-efficient \\
\hline$\varepsilon_{t-1}$ & Random error component \\
\hline$\phi(B)$ & Autoregressive operator of order ' $p$ ' \\
\hline$\theta(B)$ & Moving average operator of order ' $q$ ' \\
\hline$\sigma$ & Standard deviation of forecast errors \\
\hline$w$ and $h$ & Constants selected by simulation \\
\hline$x_{t}$ & Demand at time $\mathrm{t}$ \\
\hline$z^{*}$ & Control limit for the parabolic mask tracking signal \\
\hline$z_{t}$ & Stationary series \\
\hline
\end{tabular}




\section{Chapter 1}

\section{Introduction}

Frequently, there is a time lag between awareness of an impending event and occurrence of that event. This lead time is the main reason for planning and forecasting. If the lead time is zero or very small, there is no need for planning. If the lead time is long, and the outcome of the final event is conditional on identifiable factors, planning can perform an important role. In such situations forecasting is needed to determine when an event will occur or a need arises, so that appropriate actions can be taken. In management and administrative situations the need for planning is great because the lead time for decision making ranges from several years (for the case of capital investments) to a few days or even a few hours (for transportation or production schedules). Forecasting is an important aid in effective and efficient planning.

\subsection{Background}

Armstrong (2001) defines forecasting as "estimating in unknown situations". Predicting is a general term and connotes estimating for any time series, cross-sectional, or longitudinal data. Forecasting is commonly used when discussing time series. Martino (1983) states that technological forecasting includes four elements: the time of the

forecast or the future date when the forecast is to be realized, the technology being forecast, the characteristics of the technology or the functional capabilities of the technology, and a probabilistic statement about the forecast.

The purpose of forecasting is to reduce the risk in decision making. The magnitude of the forecasting errors experienced will depend upon the forecasting system 
used. Because forecasting can never completely eliminate risk, it is necessary that the decision process explicitly consider the uncertainty remaining subsequent to the forecast. This implies that the forecasting system should provide a description of forecast error as well as a forecast. Ideally the forecasting system should result in an estimate of the probability distribution of the variable being predicted. This permits risk to be objectively incorporated into the decision making process.

\subsection{Defining the forecasting problem}

The use of available observations from a time series to forecast its value at some future time can provide a basis for planning, control, and optimization of business applications and industrial processes. Defining a forecasting problem starts with the decision problem. Information from the forecasting process is to be used to improve the decision process. Therefore, the nature of the decisions to be made will dictate many of the desired characteristics of the forecasting system. In determining what is to be forecast, the first class of decision involves defining the variables that are to be analyzed and predicted. The level of detail required is an important consideration. Many factors influence the level of detail used: availability of data, accuracy attainable, cost of analysis, etc. In cases where the appropriate choice of variables is not clear, it is necessary to try several alternatives and select the one giving the best performance (Montgomery, Johnson and Gardiner, 1990). This is generally done during the development of the forecasting system through simulation using historical data.

The second important class of decision involves time elements such as the forecasting period, the forecasting horizon, and the forecasting interval. The forecasting period is the basic unit of time for which the forecasts are made. The forecasting horizon 
is the number of periods in the future covered by the forecast. Finally the forecasting interval is the frequency in which new forecasts are prepared. The forecasting period and horizon are usually dictated by the decision process requiring the forecast. For a forecast to be of value, the horizon must be no less than the lead-time for implementing the decision. The forecasting interval is determined by the operating mode of the data processing system that provides information on the variable being forecasted.

A third aspect of the forecasting problem relates to the required form of the forecast. It is convenient to conceive the variable of interest as being a random variable having a particular probability distribution. The decision problem requires an estimate of some characteristic of that distribution, such as the mean, median, or most probable value for use as a forecast of the variable. Or as a measure of uncertainty, the forecast demands an estimate of standard deviation, a percentile or an interval having a high probability of containing the actual value to be realized. Usually the forecast will take on of the following forms:

- An estimate of the expected value of the variable, plus an estimate of the standard deviation of forecast error, or

- An interval that has a stated probability of containing the actual future value.

\subsection{Methods of forecasting}

Forecasting situations vary widely in their time horizons, factors determining actual outcomes, types of data patterns and many other aspects. To deal with such diverse applications, several techniques have been developed. These fall into two major categories - Quantitative, and Qualitative or Technological Methods, and two minor categories - Markov approach and Indirect Methods. Quantitative methods can be 
divided into time series and causal methods, and qualitative or technological methods can be divided into exploratory and normative methods. Figure 1.1 shows the different types of forecasting techniques.

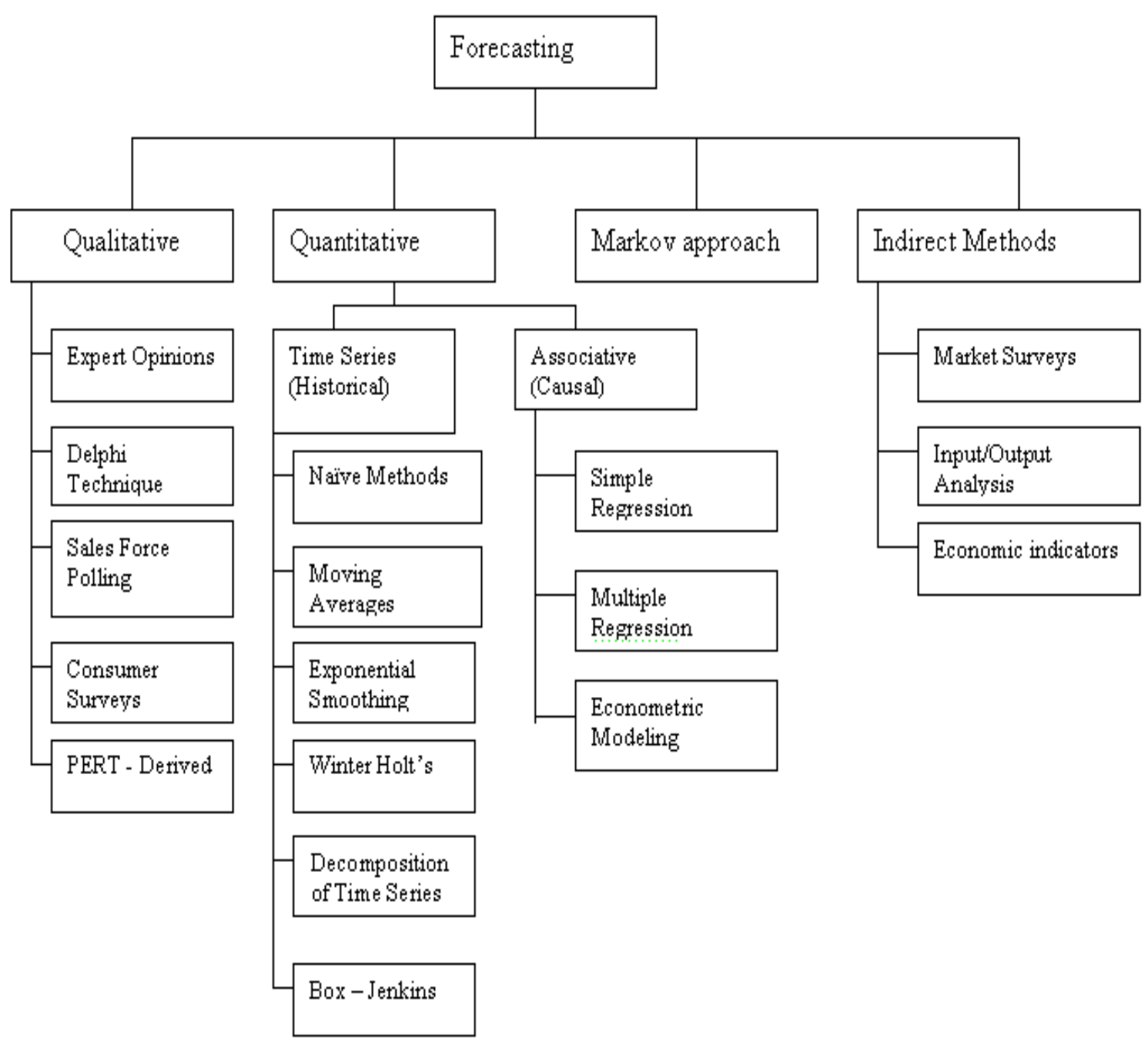

Figure 1.1 Forecasting models (Shim, Siegel, and Liew, 1994).

\subsubsection{Quantitative forecasting}

Quantitative forecasting can be applied when three conditions exist:

1. Information about the past is available.

2. This information can be quantified in the form of numerical data. 
3. It can be assumed that some aspects of the past pattern will continue into the future.

The last condition is known as assumption of continuity; it is an underlying premise of all quantitative and many technological forecasting methods no matter how sophisticated they may be.

Quantitative forecasting procedures fall on a continuum between two extremes: naïve or intuitive methods, and formal quantitative methods based on statistical principles. The first type uses horizontal, seasonal, or trend extrapolation, and is based on empirical experience that varies widely from business to business, product to product, and forecaster to forecaster. Naïve methods are simple and easy to use but not always as accurate as formal quantitative methods. Formal statistical methods can also involve extrapolation, but it is done in a standard way using a systematic approach that attempts to minimize the forecasting errors. These methods are useful when forecasts are needed for a large number of items and when forecasting errors on a single item will not be extremely costly. Another dimension for classifying quantitative forecasting methods is to consider the underlying model involved. There are two major types of forecasting models: time-series and regression (causal) models.

A time series is a time-ordered sequence of observations of a variable (Montgomery, Johnson and Gardiner, 1990). In time series, prediction of the future is based on the past values of variable and/or past errors. The objective of such time-series forecasting methods is to discover the pattern in the historical data series and extrapolate that pattern into the future. Time series analysis involves describing the processes or phenomena that generate the sequence. To forecast time series, it is necessary to 
represent the behavior of the process by a mathematical model that can be extended into the future. It is required that the model be a good representation of the observations in any local segment of time close to the present. Once a valid model for the time series process has been established, an appropriate forecasting technique can be developed. Many of the models used to represent time series are algebraic or transcendental functions of time, or some composite model that combines both algebraic and transcendental components.

Causal models exploit the relationship between the time series of interest and one or more other time series. If these other variables are correlated with the variable of interest and if there appears to be some cause for this correlation, a statistical model describing this relationship can be constructed. Then, knowing values of the correlated variables, we can use the model to obtain a forecast of the dependent variable (Montgomery, Johnson and Gardiner, 1990). An obvious limitation to the use of causal models is the requirement that the independent variables be known at the time the forecast is made. Another limitation to the use of causal models is the large amount of computation and data handling compared with certain forms of time series models.

\subsubsection{Qualitative forecasting}

The qualitative methods are appropriate when the data pattern from the past cannot be assumed to continue into the future or when the forecast is about unlikely or unexpected events in the future (Makridakis, Wheelwright, McGee, 1983). These forecasts are based on educated opinions used to predict future events subjectively. Qualitative methods, on the other hand, do not require data in the same manner as 
quantitative forecasting methods. These inputs depend on the specific method and are mainly the product of intuitive thinking, judgment, and accumulated knowledge.

Exploratory methods such as Delphi, S-curves, analogies and morphological research begin with the past and present as their starting point and move toward the future in a heuristic manner, often looking at all available possibilities.

Normative methods such as decision matrices, relevance trees, and system analysis start with the future by determining future goals and objectives, then work backwards to see if these can be achieved, given the constraints, resources and technologies available.

\subsubsection{Markov's Approach}

Markov chains, named in honor of a Russian mathematician, have been the focus of much interest by researchers. Numerous books and articles have touted Markov chains as a superior forecasting technique. Markov chains use the recent pattern of behavior as a basis for forecasting. Behavior in the future is forecasted from knowledge of the current state of an element and from an analysis of how the element moves from one state to another. The first step in using Markov chains for prediction is to develop a transition matrix. This summarizes the data by indicating the fraction of times that the behavior in one trial will change (move to another state) in the next trial. An assumption of stability is made when Markov chains are used. This means that it becomes risky to obtain longrange forecasts in cases where efforts are made to change the transition matrix. Markov chains seem reasonable for some problems. For example, they are widely used for personnel predictions. This technique and similar ones have been recommended frequently for predictions in marketing when people are assumed to go through various 
states in using a product (e.g., trial, repeat purchase, and adoption) (Armstrong. J. S., 1985).

\subsubsection{Indirect methods}

Indirect methods include techniques in which forecasts can be based on projections of national or regional economic activity (such as GDP or leading economic indicators), industry sales, or market surveys (Shim, Siegel and Liew, 1994). The three different indirect methods in practice are Economic indicators, Input-Output analysis and Market survey techniques.

Typically, the economic indicators method involves the following three steps:

1. Forecast the level of economic indicators.

2. Translate the forecast into industry sales forecast

3. Translate the industry sales forecast into company forecast.

There are three types of economic indicators: leading, coincident, and lagging.

The input-output analysis is concerned with the inter-industry or interdepartmental flows of goods or services in the economy or a company and its markets. Input-output analysis focuses on sales of each industry to firms in that industry, other industries, and other sectors such as governmental units and foreign purchases.

Market survey techniques constitute another important forecasting tool, especially for short-term projections. Designing surveys that provide unbiased and reliable information is a costly and difficult task. When properly carried out, however, survey research can provide managers with valuable information that would be unobtainable otherwise. While surveys provide an alternative to quantitative forecasting techniques, they are frequently used to supplement rather than replace quantitative analysis. 


\subsection{Performance Criteria}

There are a number of measures that can be used to evaluate the effectiveness of a forecasting system. Among the more important are forecasting accuracy, system cost, utility of output, and stability and responsiveness properties. The accuracy of a forecasting method is determined by analyzing forecast errors. While an unbiased forecast is desirable, it usually is more important that large forecast errors are rarely obtained. If the expected value of the forecast error is zero then the forecast is unbiased (Montgomery, Johnson and Gardiner, 1990). Hence a quantity such as the expected absolute error or the expected squared error is commonly used as a measure of forecast accuracy. In analyzing the accuracy of an installed forecasting method, it is common to employ a tracking signal test each period. The purpose is to determine if the forecast is unbiased. The tracking signal is a statistic computed by dividing an estimate of expected forecast error by a measure of the variability of the forecast error, such as an estimate of the mean absolute deviation of forecast error. If the forecasting system yields unbiased estimates, the tracking signals should be near zero. If the tracking signals deviate from zero by more then a prescribed amount, an investigation is made to determine if the forecasting model should be modified in order to better represent the time series process, which may have experienced a change. This form of analysis can be applied to a statistical forecast, a judgmental forecast, or a combination of both.

Cost is an important consideration in evaluating and comparing forecasting methods. There are one time costs for developing and installing the system and periodic costs for operating it. With regard to operating costs, alternative forecasting procedures 
may differ widely in the cost of data acquisition, the efficiency of computation, and the level of activity required to maintain the system.

Simulation is a useful technique for evaluating alternative forecasting methods. This can be done retrospectively using historical data. For each method, start at some prior time point and simulate forecasting period by period up to the present. Measures of forecast error can then be compared among methods.

Usually it is convenient to think of the two primary functions of a forecasting system as forecast generation and forecast control. Forecast generation involves acquiring data revise the forecasting model, producing a statistical forecast, introducing management judgment, and presenting the results. Forecast control involves monitoring the forecast process to detect out of control conditions and identify opportunities for improving forecasting performance. Tracking signal test is an essential component of the control function. The forecast control function should involve periodically summarizing forecasting performance and presenting the results. This feedback should encourage improvement in both quantitative and qualitative aspects of the system. The relationship between forecast generation and forecast control is shown in Figure 1.2.

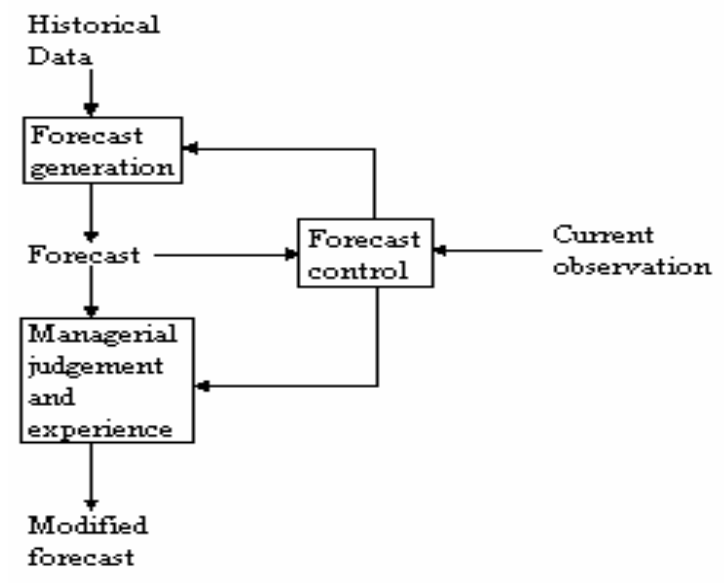

Figure 1.2 The forecasting system (Montgomery, Johnson and Gardiner, 1990) 


\subsection{Problem Definition and Need for Research}

An important prospective of forecasting is monitoring the process. Monitoring is an essential aspect of any forecasting situation (Makridakis and Wheelwright, 1989). Usually, there are two types of situations that could occur in connection with forecasting:

1. The continuation of the established patterns or relationships, and

2. Systematic changes from established patterns or relationships as time goes by.

Different monitoring approaches have been proposed in the forecasting area, among which, tracking signals test is a useful and effective detection method for identifying the occurrence of non-random changes. It can automatically detect changes in the forecasting errors when the forecast is misbehaving. Tracking signals provide quality control for forecasting (McClain, 1988).

Tracking signals test plays an even more important role when it comes to time series forecasting. This is because time series is a time-ordered sequence of observations of a variable; hence it implies that the underlying system is dynamic. When the time horizon lengthens, it is critical to monitor the process and determine if the previous pattern or relationship has been changed or not. When a nonrandom change occurs, a correspondent action needs to be taken to ensure that the forecasting process is brought back under control.

The objective of this research is to compare the ability of different types of tracking signals to detect bias and their responsiveness to non-random changes in the simulated time series. McClain (1988) concluded that the previously used performance measures are inadequate and he proposed a new criterion (ARL1: Average Run Length, i.e., average time between false alarm trips) to evaluate the performance measures. $\mathrm{He}$ 
concluded that, when measured by the new criterion (ARL1) proposed by him, the smoothed error tracking signal is better than the CUSUM method. This research extends McClain's work by evaluating the sophisticated backward CUSUM, Autocorrelation tracking signal and the parabolic mask tracking systems along with the CUSUM and smoothed error tracking signals according to McClain's criterion.

\subsection{Research Methodology}

The tracking signals will be compared by simulation using 479 time series, each series consists of monthly data of more than 100 periods in length with a constant mean and variance and random normal noise about the mean. The time series data to be used in the research is from the M-competitions which is used by hundreds of researchers. The initial values of sum of errors and the smoothed errors will be set equal to zero. The initial forecast and the Mean Absolute Deviation (MAD) will be set equal to expected values. To minimize the effects of initial values, a run-in period will be used in which no data will be collected. The length of the run-in period will be randomly generated from a uniform distribution from 15 to 45 periods. In this way, bias due to a fixed run-in interval will be minimized. After the run-in period, a step increase in the mean level will be added to each series, and the Average Run Length (ARL1) to detect the step will be measured. Control limits will be selected to yield an ARL of 100 periods at zero step size.

Different smoothing parameters will be used for the forecasting model and the tracking signal. Then, the different tracking signals will be compared according to McClain's criterion. 


\subsection{Organization}

This research will be organized in five chapters as follows: Chapter 1 gave a brief introduction to the forecasting problem, different methods of forecasting, and the forecasting model performance criteria. Chapter 2 gives some systematic literature reviews of the topics related to the research. Chapter 3 provides detailed explanation of the methodology to be used in this research. Chapter 4 consists of the results obtained using the methodology. Chapter 5 summarizes the conclusions drawn from this research and provides recommendations for future research. 


\section{Chapter 2}

\section{Literature Review}

\subsection{Time Series Models}

Time series is a collection of values observed sequentially through time (Armstrong, 2001). In time series models, historical data of the variable to be forecast are analyzed in an attempt to identify a data pattern. Then, assuming that it will continue in the future, this pattern is extrapolated in order to produce forecasts. Time series models generate predictions that are solely based on the historical pattern of the variable to be forecast. To forecast time series, it is necessary to represent the behavior of the process by a mathematical model that can be extended into the future. It is required that the model be a good representation of the observations in any local segment of time close to the present. Once a valid model for the time series process has been established, an appropriate forecasting technique can be developed. Many of the models used to represent time series are algebraic or transcendental functions of time, or some composite model that combines both algebraic and transcendental components. The idea of using a mathematical model to describe the behavior of a physical phenomenon is well established. A few forecasting models are briefly described below.

\subsubsection{Naïve methods}

Naïve method uses the most recent information available concerning the actual value. Thus, if a forecast were being prepared for a time horizon of one period, the most 
recent actual value would be used as the forecast for the next period (Makridakis, Wheelwright, McGee, 1983).

\subsubsection{Moving Average methods}

The moving average method seeks to smooth out past data by averaging the last several periods and projecting forward. The moving average operation forecasts demand by calculating an average of actual demands from a specified number of prior periods, $\mathrm{N}$. Selection of N (total number of periods used in the average) is an important aspect of this method. A higher value of $\mathrm{N}$ results in greater smoothing, and less susceptible the forecast is to random variations.

\subsubsection{Simple Moving Average}

At time $\mathrm{T}$, the $\mathrm{N}$-period simple moving average is

$$
M_{T}=\frac{x_{T-N+1}+\ldots+x_{T-1}+x_{T}}{N}
$$

$x_{T}$ is the observation at time $\mathrm{T}$, and $\mathrm{N}$ is the span of the moving average.

A large value of $\mathrm{N}$ is appropriate if the underlying pattern of demand is stable. Where as, smaller values of $\mathrm{N}$ are appropriate if the underlying pattern is changing or if it is important to identify short term fluctuations.

\subsubsection{Double Moving Average}

This method can be seen as 'moving average of the moving averages'. Here the moving average is applied twice, smoothing the already smoothed series. At time $\mathrm{T}$, the double moving average is given as

$$
M_{T}^{[2]}=\frac{M_{T-N+1}+\ldots+M_{T-1}+M_{T}}{N}
$$




\subsubsection{Exponential Smoothing}

Exponential smoothing is probably the most widely used class of procedures for smoothing discrete time series in order to forecast the immediate future. Its wide usage can be attributed to its simplicity, its computational efficiency, ease of adjusting its responsiveness to changes in the process being forecast, and its reasonable accuracy (Montgomery, Johnson and Gardiner, 1990).

\subsubsection{Simple Exponential Smoothing}

In this method the past data weights decrease exponentially with time, that is, the more recent the data value, the greater its weight. This method is best suited to data that exhibit no trend. Estimates of the parameter of the underlying time series model are updated recursively each period, as soon as the new observation for that period is available. This method reacts quickly to changes in demand. The simple exponential smoothing statistic is defined as

$$
S_{T}=\alpha x_{T}+(1-\alpha) S_{T-1}
$$

$S_{T}$ is the smoothed value or the smoothed statistic at the end of period T, $\alpha$ is the smoothing constant $(0<\alpha<1), x_{T}$ is the observation at time period $\mathrm{T}$.

Essentially the response of the forecast to changes is a function of $\alpha$. The smaller the value of $\alpha$, the slower is the response. Large values of $\alpha$ cause the smoothed value to react quickly, not only to real changes but also to random fluctuations. The effect of $\alpha$ on responsiveness can be judged by comparing exponential smoothing and moving average methods. 


\subsubsection{Double Exponential Smoothing}

This method is useful where historic data series is expected to change linearly with time. In this, single exponential smoothing method is first applied to the data and applied again to the smoothed statistic.

$$
\begin{aligned}
& S_{T}=\alpha x_{T}+(1-\alpha) S_{T-1} \\
& S_{T}^{[2]}=\alpha S_{T}+(1-\alpha) S_{T-1}^{[2]}
\end{aligned}
$$

$S_{T}^{[2]}$ implies double exponential smoothing or second order exponential smoothing.

\subsubsection{Decomposition method}

The decomposition method is the strongest available method for dealing with cyclical components (O’Donovan, 1983). Whenever there is a seasonal or cyclical trend, the Decomposition method can be used for dealing with seasonal, trend, cyclical and random error components together. The first three are estimated and used to forecast future values. This method may be used to fit the time series data, whether it is monthly, quarterly, or annually. The types of time series data where this method is applicable are sales earnings, cash flows, market share, and costs. The multiplicative form of a time series is assumed to have four components:

$$
X_{t}=I_{t} * C_{t} * T_{t} * E_{t}
$$

$X_{t}$ is the time series value at time $\mathrm{t}, I_{t}$ is the Seasonal component (or Index) at time t, $T_{t}$ is the Trend component at time $\mathrm{t}, C_{t}$ is the Cyclical component at time $\mathrm{t}$ and $E_{t}$ is the Random error component.

Seasonal effect of the time series is calculated by: 


$$
I_{t} * E_{t}=\left(\frac{X_{t}}{T_{t} * C_{t}}\right)
$$

The seasonal factors for each season are averaged and adjusted since the multiplicative model requires that the average seasonal factors equal 1.0. Thus, the demand for each period is multiplied by the seasonal factor for that period to remove seasonality. De-seasonalized data are then used to determine a regression equation to generate the forecast.

\subsubsection{Winter's Model}

This method is a more sophisticated version of exponential smoothing in which allowance is made for trend and seasonal patterns in the data. This method is often used for immediate and short term forecasts, since it is quicker to prepare, has lower data requirements, is easier to understand, and less costly. This multiplicative model adjusts a given forecast by multiplying the forecast by a seasonal factor. In this model an average seasonal factor is calculated taking into account the average demand per period. In this method the trend is smoothed separately. This helps with accuracy and flexibility.

$$
\begin{aligned}
& S_{t}=\alpha x_{t}+(1-\alpha)\left(S_{t-1}+b_{t-1}\right) \\
& b_{t}=\gamma\left(S_{t}-S_{t-1}\right)+(1-\gamma) b_{t-1} \\
& F_{t+m}=S_{t}+b_{t} * m
\end{aligned}
$$

Equation (2.7) adjusts $S_{t}$ for the trend of the previous period $\left(b_{t-1}\right)$ by adding it to the last smoothed statistics $S_{t-1}$. This offsets the lag in $S_{t}$ and brings it up to the base of the current data value. Equation (2.8) updates the trend. Equation (2.9) is used for forecasting m periods ahead, where $F_{t+m}$ is the $m$-period ahead forecast made at time $t, \alpha$ is the 
smoothing constant, $(0<\alpha<1), S_{T}$ is the smoothed statistic, $x_{t}$ is the observation in period $\mathrm{t}, b_{t}$ is the trend component, and $\gamma$ is the trend smoothing coefficient, assumed value between 0 and 1.

\subsubsection{Box - Jenkins models}

Box-Jenkins forecasting models are based on statistical concepts and principles and are able to model a wide spectrum of time series behavior. It has a large class of models to choose from and a systematic approach for identifying the correct model form. There are both statistical tests for verifying model validity and statistical measures of forecast uncertainty. In contrast, traditional forecasting models offer a limited number of models relative to the complex behavior of many time series with little in the way of guidelines and statistical tests for verifying the validity of the selected model.

Box-Jenkins method is used where immediate and short term forecasting with most accurate forecasts is desired (O'Donovan, 1983). The Box-Jenkins Auto Regressive Integrated Moving Average (ARIMA) model, like most statistical forecasting methods, extrapolates past pattern within a single data series into the future. Time series data refer to observations on a specific variable that occur in a time sequence. One of the important aspects of the ARIMA time series analysis is the parsimony of the model.

A univariate ARIMA model is composed of two terms, which are an Auto Regressive (AR) term and a Moving Average (MA) term. The basic form of the model is shown as follows (Box and Jenkins, 1976):

$$
\phi(B) \nabla^{d} z_{t}=\theta(B) \varepsilon_{t}
$$


Where $\phi(B)$ is the autoregressive operator of order ' $p$ '; $\nabla^{d}$ is the backward difference operator of order ' $d$ '; $\mathrm{z}_{t-1}$ is the stationary series; $\theta(B)$ is the moving average operator of order ' $q$ '; $\varepsilon_{t-1}$ is the random error component; the two functions are based on the statistical relationship between the available data. The variable $z$ in the second term is directly related to one or more part of the series values, while the variable $a$ in the third term is related to one or more random errors. The random errors are determined by the differences between the predicted values and the observed values at a given time period.

The ARIMA model can be described in a simple format such as ARIMA $(p, d, q)$. The ' $p$ ' is the number of auto-regressive parameters in the model, while the ' $q$ ' is the number of the moving average parameters. If the time series is nonstationary, the term ' $d$ ' should be included in the model. The stationarity of the time series means that it fluctuates randomly about a constant mean. A three step iterative procedure is used to build an ARIMA model. First a tentative model of the model of the ARIMA class is identified through the analysis of historical data. Then the unknown parameters of the model are estimated. Finally, diagnostic checks are performed to determine the adequacy of the model, or to indicate each of these steps in more detail (Montgomery, Johnson and Gardiner, 1990).

\subsubsection{Identification}

Tentative identification of an ARIMA time series model is done through analysis of actual historical data. The primary tool used in the identification process is the autocorrelation function. The partial autocorrelation function also proves useful in the identification process. Once the sample autocorrelation and partial autocorrelation functions are computed, they are exhibited on the graph and a tentative model is 
identified by comparing observed patterns with theoretical autocorrelation function patterns. If the time series is nonstationary, the usual approach is to compute the sample autocorrelation and partial autocorrelation function for the first difference of the series. If these functions behave according to the theoretical patterns then one difference is necessary to produce stationarity. If not, successively higher order differencing should be applied until stationary behavior is achieved. If the differenced series has a non-zero mean, at the identification stage we may obtain an indication of whether or not a nonzero value is needed by comparing the sample mean of the differenced series with its approximate standard error. Box and Jenkins (1976) give the approximate standard error for several useful ARIMA $(p, d, q)$ models. Several useful examples of the identification process are given in Box and Jenkins (1976) and Nelson (1973).

\subsubsection{Estimation}

After an appropriate time series has been tentatively identified, the least squares estimates of the model parameter are obtained. If a model is linear, then the partial derivative of the random error component with respect to any parameter is not a function of the model parameters. If the preliminary parameter estimates are not statistically efficient, we should be cautious in drawing inferences from such a model, as the final least squares estimates of the model parameters may differ considerably from the initial values (Montgomery, Johnson and Gardiner, 1990).

\subsubsection{Diagnostic Checking}

Once a tentative model has been fit, the model adequacy should be examined, and, if necessary potential improvements should be suggested. If the fitted model is 
adequate, it should transform the observations into a white noise process. Thus, a logical method of diagnostic checking is to compute the residuals and then estimate and examine their autocorrelation function. If the model is appropriate, then the residual sample autocorrelation should have no structure to identify; that is, the autocorrelation should not differ significantly from zero for all lags greater than one. If any of the residual autocorrelations are non zero, we would incorporate the apparent structure into the original model and refit the series. This process is continued until the residual autocorrelations resemble those of a white noise process.

The model is used for short term and intermediate term forecasting, updated as new data becomes available to minimize the number of periods ahead required of the forecast.

\subsection{Tracking Signals}

In most forecasting systems, it is important to automatically monitor the forecast errors to ensure that the system remains in control. Tracking signal test is a procedure which is used to automatically determine if a pattern or relationship has changed or not and more importantly, when it has changed, it leads to the action to bring the process back in control. Gardner (1985) classified monitoring procedures into five categories:

1. Simple CUSUM (CUmulative SUM) tracking signals,

2. Smoothed error tracking signals,

3. Backward CUSUM control systems,

4. Autocorrelation tracking signals, and

5. Parabolic mask tracking systems. 
The first two are used most often. Tracking signals in general are ratios. The numerator is a weighted or unweighted sum of forecast errors, while the denominator is usually the mean absolute deviation (MAD), which is defined as:

$$
M A D=\frac{1}{n} \sum_{i=1}^{n}\left|e_{i}\right|
$$

The numerator has an expected value of zero when the forecast is in control, and would move away from zero if the forecast goes out of control. The more the forecasting errors vary from zero in some systematic way, the greater the chances that the established pattern or relationship being monitored has changed.

\subsubsection{The simple CUSUM tracking signal}

The simple CUSUM tracking signal was proposed by Brown (1959). This signal compares the cumulative sum (CUSUM) of the errors at the end of each time period with a smoothed value of the mean absolute deviation (MAD). If the ratio CUSUM/MAD exceeds a pre-specified limit, the forecasting approach is reexamined to see whether the pattern has changed and whether some action needs to be taken. The updating equations as each new error is observed are:

$$
\begin{aligned}
& e_{t}=X_{t}-F_{t} \\
& S U M_{t}=e_{t}+S U M_{t-1} \\
& M A D_{t}=\alpha\left|e_{t}\right|+(1-\alpha) M A D_{t-1} \\
& \text { CUSUM }_{t}=\left|S U M_{t} / M A D_{t}\right|
\end{aligned}
$$

The forecast error, $e_{t}$, is the actual time series value, $X_{t}$, minus the forecast $F_{t}$.

The smoothing parameter, $\alpha$, is restricted to $0 \leq \alpha \leq 1$. 


\subsubsection{Control limits for simple CUSUM:}

Gardner (1983) suggests two approaches to the problem of selecting control limits for $\operatorname{CUSUM}_{t}$ as well as other tracking signals.

1. To use a control limit that yields some desired probability of getting a 'false trip', defined as Type I error, or a case where the control limit is exceeded due to chance rather than biased forecasts.

2. To base the control limit on the number of time periods required to detect biased forecast errors of any given size.

\section{Control limits for simple CUSUM using probabilities:}

In this approach, the normal distribution is often used to find the probability of a false trip. For example, Brown (1963) shows that the simple CUSUM for exponential smoothing is approximately normally distributed, with standard deviation equal to

$$
\sigma_{c}^{\sim}=\frac{0.884}{\sqrt{\alpha}}
$$

Gardner (1983) tested the validity of normal approximation for CUSUM ${ }_{t}$ based on Equation (2.16) by simulation. Table 2.1 gives the simulated distributions of CUSUM from simple exponential smoothing forecasts (one period ahead) for $\alpha=0.1,0.2$, and 0.3 . He observed that the normal approximation for $C_{U S U M}$ with standard deviation equal to Equation (2.16) agrees very well with the simulated distribution for $\alpha=0.1$ in Table 2.1. However, there are some differences at higher $\alpha$ values. This should be expected, since the assumptions used to develop Equation (2.16) hold only for small $\alpha$ values. It may also be observed that the control limits at any probability level decrease as $\alpha$ is increased. One reason for this effect is that the increase in $\alpha$ increases the MAD. Another reason is that 
exponential smoothing models have a tendency to induce negative autocorrelation in the errors from one period ahead forecast.

Table 2.1 Cumulative distribution of simple CUSUM tracking signal (Gardner, 1983)

\begin{tabular}{|c|c|c|c|}
\hline cumulative & & CUSUM $_{t}$ & \\
\cline { 2 - 4 } Probability & $\alpha=0.1$ & $\alpha=0.2$ & $\alpha=0.3$ \\
\hline 0.8 & 3.6 & 2.6 & 2.1 \\
\hline 0.9 & 4.7 & 2.9 & 2.8 \\
\hline 0.95 & 5.6 & 4.1 & 3.5 \\
\hline 0.96 & 5.9 & 4.3 & 3.7 \\
\hline 0.97 & 6.3 & 4.6 & 3.9 \\
\hline 0.98 & 6.8 & 5 & 4.3 \\
\hline 0.99 & 7.5 & 5.6 & 4.9 \\
\hline
\end{tabular}

\section{Control limits for simple CUSUM using average run lengths:}

This approach is based on the concept of average run length (ARL) to detect biased forecast errors. McClain (1988) suggests the following two definitions for ARL.

1. ARL is the average time between false alarm trips in an operating forecasting system (ARL 1).

2. ARL is the average length of time until a false alarm trip occurs, starting from an arbitrarily selected point in time (ARL 2).

Control limits were determined based on simulation. Gardner $(1983,1985)$ designed the control limits based on the criterion that ARL 2 be 100 for false alarms. McClain's (1988) design criterion was, whenever the tracking signal exceeded the control limit, the sum of errors was reset to zero and the time since the last reset was recorded. However, the forecasts were not reset after a trip, since the false alarms are being simulated. McClain (1988) observed that the difference between ARL1 and ARL2 for CUSUM was large. 


\subsubsection{Advantages and Disadvantages of simple CUSUM:}

Compared to other tracking signals, the simple CUSUM has two advantages (Gardner 1983). First, the simple CUSUM performance is independent of smoothing parameter used. The performance of tracking signals based on the smoothed error or autocorrelation deteriorates badly as the smoothing parameter is increased. Second, the performance of the simple CUSUM is also independent of the variance of the time series. If the variance of the series changes, this is estimated implicitly by $\mathrm{MAD}_{\mathrm{t}}$. Both $\mathrm{SUM}_{\mathrm{t}}$ and $\mathrm{MAD}_{\mathrm{t}}$ will change proportionately, leaving the ARL performance unchanged. The simple CUSUM is effective for detecting small shifts (Adams and Lin, 1996).

The simple CUSUM also has some disadvantages, as Trigg (1964) and numerous others have pointed out, that the simple CUSUM never forgets large errors. Once the tracking signal has gone out of limits it will not necessarily return within limits even though the forecasting system itself comes back in control. Consequently, intervention is necessary to set the sum of the errors back to zero if future false alarms are to be avoided. Such interventions can be tedious. Another disadvantage is ironic. If the forecasting system starts to give exceptionally accurate forecasts, the signal may trip. Suppose that near perfect forecasts begin to occur. This will cause the MAD to tend to zero, leaving the CUSUM unchanged. Thus CUSUM $t$ will tend to infinity.

\subsubsection{The backward CUSUM control systems:}

The backward CUSUM control systems approach was developed by Harrison and Davies (1963). It is based on the notion that if a past change in a time series could be guessed, then the sum of all errors since the change would be the best tracking signal available. Since there is no way of knowing the number of past periods to sum in 
advance, it is necessary to store the information of all possible CUSUMs. The first one is the last error; the second is the sum of the last two errors, and so on to the beginning of the series. Each of these sums should be the most sensitive signal available to detect a change in the time series that occurred $i$ periods ago, where $i$ is the number of periods used in each CUSUM. However, the number of such CUSUMs could quickly get out of hand in any time series, but the basic idea can be modified to a more practical scheme. One modification is to maintain only the last six to twelve CUSUMs since the information on older changes is not likely to be of much value at present. Control limits are setup in linear form, as

$$
L_{i}=\sigma w(i+h)
$$

Where $L_{i}$ is the limit (+ or -) on the $i$ th backward CUSUM, $\sigma$ is the standard deviation of forecast errors computed during a period when the system is in control, $w$ and $h$ are constants selected by simulation.

\subsubsection{Implicit tests for bias:}

The storage requirements for six CUSUMs and their control limits would still be burdensome in most forecasting systems. To reduce the storage requirements, Harrison and Davies (1964) devised a system whereby all CUSUMs can be implicitly tested against their control limits by storing only four quantities. Proof of this result may be found in Harrison and Davies (1964) or Coutie et al. (1966). Two of the four quantities are constants: $\sigma w$ and $L_{0}=\sigma w h$. Since the limits increase by $\sigma w$ each period, these two quantities are sufficient to compute each $L_{i}$. The other two quantities are moving parameters. The parameter used to check for positive bias is the minimum of the differences between the positive control limits and their corresponding CUSUMs, $L_{i}-S_{i}$, 
from the last period. The other parameter used to check for negative bias, is the maximum of the differences between the negative control limits and their CUSUMs is $L_{i}-S_{i}$

The test for positive bias is

$$
D_{t}^{+}=\operatorname{MIN}\left[D_{t-1}^{+}, L_{0}\right]+\sigma w-e_{t}
$$

$D_{0}^{+}$is set equal to $L_{0}$. If $D_{t}^{+}<0$, lack of control is signaled.

The test for negative bias is

$$
D_{t}^{-}=\operatorname{MAX}\left[D_{t-1}^{-},-L_{0}\right]-\sigma w-e_{t}
$$

$D_{0}^{-}$is set equal to $-L_{0}$. If $D_{t}^{-}<0$, lack of control is signaled.

\subsubsection{Control limits for the backward CUSUM system:}

Finding control limit parameters ( $w$ and $h$ ) for the backward CUSUM system is a tedious process. The only published control limits for forecast errors that are based on a large sample size, with a run in period to wash out initial conditions, are those given by Golder and Settle (1976). Their limits do not yield even multiples of ARLs on unbiased errors, making it difficult to compare the backward CUSUM to other tracking signals. For reasons explained in their paper, Golder and Settle show different sets of control limits to detect different levels of bias, making it impossible to study the effects of varying the $w$ and $h$ parameters.

\subsubsection{Advantages and disadvantages of the backward CUSUM system:}

Although the backward CUSUM is the most thorough tracking signal available, it has not been used in practice for a number of reasons (Gardner, 1983). The first reason is the lack of published control limits. The second is the lack of comparisons to other 
tracking signals. Third, the other tracking signals considered in this research require storage of only two quantities, where as backward CUSUM requires storage of four quantities. It can be a huge disadvantage in large forecasting systems. Fourth, the backward CUSUM requires the assumption of a constant variance in the time series. This is a disadvantage in setting up a control system for a time series with a short history. Also, the performance of the backward CUSUM can be erratic if the true variance of the series differs significantly from the estimated variance.

\subsubsection{The smoothed error tracking signal:}

The smoothed error tracking signal was developed by Trigg (1964) in an attempt to overcome the disadvantages of the long memory of the simple CUSUM. Rather than taking the ratio of the actual CUSUM to the smoothed MAD, Trigg's method uses an exponentially weighted smoothed value of the forecast error divided by a smoothed value of the MAD. If the ratio exceeds a pre-specified limit, it signals that probably a change in pattern or relationship has occurred.

The smoothed error tracking signal has been widely used in practice. It consists of two smoothing equations and the tracking signal $S E T S_{t}$. It requires the continued updating and storage of two values, $E_{t}$ and $M_{t}$. The updating equations are as follows:

$$
\begin{aligned}
& E_{t}=\alpha e_{t}+(1-\alpha) E_{t-1} \\
& M A D_{t}=\alpha\left|e_{t}\right|+(1-\alpha) M A D_{t-1} \\
& \text { SETS }_{t}=\left|E_{t} / M A D_{t}\right|
\end{aligned}
$$

where $e_{t}=X_{t}-F_{t}$, and $\alpha$ is a smoothing parameter whose value is between 0 and 1 . 
The above equations can be simplified by recognizing that exponential smoothing recurrence relations are basically approximations to exact discounted averages (Gilchrist, 1967, 1976). The simplified equations are as follows:

$$
\begin{aligned}
& E_{t}=e_{t}+\beta E_{t-1} \\
& \text { MAD }_{t}=\left|e_{t}\right|+\beta M A D_{t-1} \\
& \text { SETS }_{t}=\left|E_{t} / M A D_{t}\right|
\end{aligned}
$$

where $\beta$ is the discount factor, restricted to $0 \leq \beta \leq 1$.

The value of the tracking signal SETS $S_{t}$ is exactly the same when using exponential smoothing or discounted averages, provided that the same starting values are used and $\beta=1-\alpha$.

Once a forecasting system is in operation, $e_{t}$ is known, $E_{t}$ and $M_{t}$ will be computed, thus the tracking signal ratio $S E T S_{t}$ can be obtained. If the value of $S E T S_{t}$ is close to zero, i.e., $M_{t}$ is much larger than $E_{t}$, it implies that the errors are random. When some systematic change occurs, the forecast errors will be consistently positive or negative, increasing the value of $E_{t}$, which will cause the ratio $S E T S_{t}$ to increase. If a certain limit is reached, it implies that the errors have ceased to be random; therefore the forecasting procedure needs to be reexamined.

\subsubsection{Control limits for the smoothed error signal:}

A lot of conflicting analytical and simulation work has been done on the probability distribution of SETS . Trigg's original distribution (1964) is applicable only to forecasting models other than exponential smoothing, since he ignores the autocorrelation in errors caused by exponential smoothing. Brown (1967) took this autocorrelation into 
account in developing distributions for exponential smoothing. Batty (1969), working independently of Brown, developed somewhat different distributions for exponential smoothing. Gardner's (1983) simulated distributions agree with Trigg for independent errors and Batty for exponential smoothing.

\subsubsection{Advantages and disadvantages of the smoothed error signal:}

The smoothed error signal is not recommended for higher values of $\alpha$. The ARLs at $\alpha=0.2$ are significantly larger than at $\alpha=0.1$ for levels of bias up to $2 \sigma$, (Gardner, 1983). The reasons for this deterioration in performance are complex. Increasing $\alpha$ causes the smoothed error to increase faster than the smoothed MAD. The tracking signal finds it harder to distinguish between bias and purely random fluctuations in the time series as a result of this.

Although the smoothed error signal works well on independent errors, the autocorrelation signal is a better choice. As in the case of simple CUSUM, the performance of the smoothed error signal based on smoothed MAD is independent of the variance of the time series. The smoothed error signal and the simple CUSUM provide much larger than anticipated ARLs when the forecast residuals are negatively correlated and when forecast residuals are positively correlated, the smoothed error simple CUSUM provide smaller than anticipated ARLs (Adams and Tseng, 1998).

\subsubsection{The autocorrelation tracking signal:}

It is important to check the forecast errors for the occurrence of autocorrelation between successive errors. Ideally the errors will be independent. In order to indicate any large local autocorrelation as well as bias, there is a need for tracking signal. Gilchrist 
(1976) proposed a simple tracking signal based on the Von-Neuman ratio. It is based on mean square successive difference. The autocorrelation tracking signal is the ratio of the discounted mean square successive difference to the discounted mean square error. The numerator and denominator can be evaluated using the recurrence relations. Gardner (1983) developed a tracking signal for autocorrelation defined as the ratio of the smoothed covariance in errors (at lag 1) to the smoothed MSE. The updating equations are as follows:

$$
\begin{aligned}
& C O V_{t}=e_{t} e_{t-1}+\beta C O V_{t-1} \\
& M S E_{t}=e_{t-1}^{2}+\beta M S E_{t-1} \\
& R T S_{t}=C O V_{t} / M S E_{t}
\end{aligned}
$$

where $C O V_{t}$ is the covariance at time t, $M S E_{t}$ is the Mean Squared error at time t, $e_{t}=X_{t}-$ $F_{t}, R T S_{t}$ is the tracking signal, $\beta$ is the discount factor, restricted to $0 \leq \beta \leq 1$.

\subsubsection{Control limits for the autocorrelation signal:}

The control limits for the autocorrelation signal are best found using simulations of both independent errors and of errors showing autocorrelations of the various magnitudes. Gardner (1983) published the control limits based on simulations where the distribution was symmetric about zero. Thus, control limits can be set for both positive and negative autocorrelation or for positive autocorrelation only, which corresponds to bias. If the MSE is smoothed, the tracking signal is independent of variance.

\subsubsection{Advantages and disadvantages of the autocorrelation signal:}

The autocorrelation signal is the only signal which can be used to track both positive and negative autocorrelation in forecast errors (Gardner, 1983). This ability is 
useful in fitting adaptive filtering or adaptive estimation procedure models to historical data. The autocorrelation signal is not recommended for exponential smoothing models because of the autocorrelation induced by the forecasting process. $R T S_{t}$ spends most of its time below zero on exponential smoothing errors, uselessly chasing negative autocorrelation. If $R T S_{t}$ is negative when a step change in time series occurs, it will lag behind other signals in sounding the alarm unless the step is quite large. Adams and Tseng (1998) have shown that substantial sample sizes are required for estimating the parameters and that updating and validating the parameter estimates would be prudent. Another disadvantage of the autocorrelation signal applies to independent forecast errors as well as errors from exponential smoothing.

\subsubsection{The parabolic mask tracking system:}

One disadvantage with simple CUSUM is that the CUSUM may wander away from zero over time due to nothing more than randomness. To mitigate this problem Brown $(1971,1982)$ developed the parabolic mask tracking system which operates using the record of last 8-12 simple CUSUM values. To test for bias, a parabola is constructed and centered over the most recent CUSUM. The arms of the parabola point backward in time. If all preceding sums stay within the arms of the parabola, the system is in control. If any previous CUSUM falls outside the area of the parabola, the system is out of control.

$$
\operatorname{CUSUM}_{j}=\sum_{i=1}^{j} x_{i}, j=1,2, \ldots
$$

$\mathrm{CUSUM}_{j}$, in Equation (2.29) can be written as 


$$
\begin{aligned}
\operatorname{CUSUM}_{j} & =\sum_{i=1}^{j} x_{i} \\
& =\sum_{i=1}^{n} x_{i}-\sum_{i=j+1}^{n} x_{i} \quad 1 \leq j \leq n \\
& =\text { CUSUM }_{n}-\sum_{i=j+1}^{n} x_{i}
\end{aligned}
$$

The following parabolic limits that should be plotted against CUSUMj $(1<t<\mathrm{n}-1)$ :

$$
\begin{aligned}
& \operatorname{CUSUM}_{j} \geq z^{*} \sigma \sqrt{(n-j)}+\operatorname{CUSUM}_{n} \\
& \operatorname{CUSUM}_{j} \leq-z * \sigma \sqrt{(n-j)}+\operatorname{CUSUM}_{n}
\end{aligned}
$$

The $\mathrm{z}^{*}$ in Equations (2.31) is chosen such that the CUSUM achieves a pre-specified incontrol ARL.

The threshold is governed by the width of the parabola, called the latus rectum. The idea is very large biases are detected quickly. Smaller, but consistent, biases take longer to detect. The underlying theory comes from Wald's sequential analysis. This should be a more powerful statistical test because the distance from zero of the last CUSUM is irrelevant - as only recent changes in CUSUM are tested. Unlike the tabular form of CUSUM charts which involves formulae, the parabolic CUSUM is easier to implement and understand (Atienza, Tang, Ang, 2000).

\subsubsection{V-masks:}

Suppose null hypothesis says that the errors are random samples from a population with mean $\mu_{0}=0$, which indicates that the forecasts are unbiased. An alternative hypothesis is that these errors come from some other population which is biased by an amount say $\mu_{1}=\mathrm{k} \sigma$. Both populations are assumed to be normal and have 
the same variance $\sigma$. It should also be tested to find whether the forecasts were biased in the opposite direction $\mu^{\prime}=-k \sigma$. Hence the acceptance chart has V-shaped decision regions. The forecast should be continued to see more information is required to decide that the forecasts are biased, or whether we conclude that the forecasts are unbiased.

\subsubsection{Parabolic masks:}

The parabolic mask is the envelope over the parameter $\mathrm{k}$ of all the V-masks. Let the risk of accepting the alternative hypothesis when the population mean is really $\mu_{0}$ be designated by $\alpha$ and the risk of accepting the null hypothesis when the population mean is really be $\mu_{1}$ designated by $\beta$. Wald's (1947) book showed that the critical limits should be approximately

$$
\lambda_{0} \cong(1-\alpha) / \beta \text { and } \lambda_{1} \cong \alpha /(1-\beta)
$$

Since we have taken $\mu_{0}=0$ and $\mu_{1}= \pm \mathrm{k} \sigma$, the equations of the two limits for the Wald acceptance region are:

$$
\frac{ \pm \sigma^{2} \ln \lambda_{1}}{k \sigma}+\frac{n k \sigma}{2}=\frac{ \pm \sigma \ln (1-\beta) / \alpha}{k}+\frac{n k \sigma}{2}
$$

Where $\mathrm{n}$ is the sample size. If the actual change were large ( $\mathrm{k}$ large), then the intercept of the V-mask is small and the slope large; detection occurs early, with little evidence conversely, when the actual change to be detected is quite small (k small), the intercept is quite large so that such a change is impossible to detect quickly, but the slope of the arms of the V- mask is small. Eventually a small change would be detected. The point of the parabolic envelope is that it will detect any size change, with specified risks. If the use of the tracking signal determined the size change that was important, we could use the Vmask. The envelope of all $\mathrm{V}$ - masks over the parameter $\mathrm{k}$ is a parabola 


$$
S(n)=\sigma \sqrt{2 \ln (1-\beta) / \alpha} \sqrt{n}
$$

The Parabolic CUSUM provides rather good detection for almost any size of a shift. This can sometimes be regarded as undesired property, since it will give alarm signals also for deviations from target value of negligible size. An important point is that such an alarm from Parabolic CUSUM is likely to be based on a rather long run of observations, hence giving an indication that the shift is not an important one (Stig, 1997).

\subsubsection{Empirical comparisons:}

There have been a few empirical studies comparing the ability of tracking signals to detect bias in the forecast errors. The first was by Golder and Settle (1976), who found that Harrison and Davies' backward CUSUM was superior to Trigg's signal. Gardner (1983, 1985) compared the ability of all five signals to detect bias in simulated time series. Surprisingly, the simple CUSUM was recommended as the best signal. The simple CUSUM gave about the same performance as the sophisticated CUSUM models and performed significantly better than the Trigg and autocorrelation signals. Gardner (1985) found that using a relatively small parameter in the Trigg signal improved performance as McKenzie predicted. However, the signal was unstable and unresponsive to bias at any smoothing parameter (in the forecasting model) above 0.1, regardless of the parameter used in the tracking signal. McClain (1988) concluded that the previous performance measures were inadequate and he proposed a new criterion to evaluate the performance measures. He found that when measured by the new criteria, the smoothed error tracking signal is substantially better than simple CUSUM method. The Table below compares the work done by the researchers on different types of tracking signals. 
Table 2.2 Empirical Comparisons

\begin{tabular}{|l|c|c|c|c|c|c|c|}
\hline & \multicolumn{4}{|c|}{ Tracking signals } & \multicolumn{2}{c|}{ Comparison Crite rion } \\
\cline { 2 - 8 } & CUSUM & $\begin{array}{c}\text { Backward } \\
\text { CUSUM }\end{array}$ & $\begin{array}{c}\text { Smoothed } \\
\text { error }\end{array}$ & $\begin{array}{c}\text { Auto } \\
\text { correlation }\end{array}$ & $\begin{array}{c}\text { Parabolic } \\
\text { Mask }\end{array}$ & ARL 1 & ARL2 \\
\hline Golder and Settle & & $\sqrt{ }$ & $\sqrt{ }$ & & & & $\sqrt{ }$ \\
Gardner & $\sqrt{ }$ & $\sqrt{ }$ & $\sqrt{ }$ & $\sqrt{ }$ & & & $\sqrt{ }$ \\
McClain & $\sqrt{ }$ & & $\sqrt{ }$ & & & $\sqrt{ }$ & \\
Current work & $\sqrt{ }$ & $\sqrt{ }$ & $\sqrt{ }$ & $\sqrt{ }$ & $\sqrt{ }$ & $\sqrt{ }$ & \\
\hline
\end{tabular}

\subsection{Adaptive control of smoothing parameters}

Several techniques have been developed to monitor and modify automatically the value of smoothing constant in exponential smoothing. These techniques are usually called adaptive control smoothing methods, because the smoothing parameter modifies or adapts itself to changes in the underlying time series. Some measure of forecast accuracy is monitored to detect changes in the series. If recent accuracy has been good, adaptive control systems assume that the structure of the time series is stable and should apply moderate parameters. If accuracy deteriorates, the assumption is that the structure of the series has somehow changed and the parameters are increased in order to shorten the response lag in the forecasts. The next paragraph compares a number of methods for controlling adaptive parameters.

The most popular control method is based on Trigg's tracking signal described before (see also Trigg and Leach, 1967). As each error is observed, this method sets the value of $\alpha_{t}$, equal to the value of the smoothed forecast error divided by the MAD. Chow (1965) developed another control method called evolutionary operation. This approach requires that three forecasts be computed each period. One forecast is computed using a base value of a. The others are computed using $\alpha_{H}=\alpha+0.05$ and $\alpha_{L}=\alpha-0.05$. If the forecast error using $\alpha$ is less than that using $\alpha_{H}$ and $\alpha_{L}$, no change in $\alpha$ is made. If the error 
from $\alpha_{H}$ or $\alpha_{L}$ is lower, $\alpha$ is reset to $\alpha_{H}$ or $\alpha_{L}$. New values of $\alpha_{H}$ and $\alpha_{L}$ are computed and the process begins afresh. Chow's work was extended by Roberts and Reed (1969), and later by Montgomery (1970), to automatically control two or more parameters in the same model. The stability of these control methods has been widely criticized (Fildes, 1979). Several modifications have been suggested in an attempt to avoid unstable forecasts. Shone (1967) suggested that the changes in $\alpha$ using the Trigg and Leach method be delayed one period - that is, we set $\alpha$ equal to the value of the ratio at $t-l$. Flowers (1980) suggested that the range of permissible values for $\alpha$ be constrained. Whybark (1973) developed a conservative model which changes $\alpha$ only when certain control limits on the size of each error are broken. Dennis (1978) developed a similar model which changes $\alpha$ only when a control limit on the number of consecutive errors with the same sign has been broken. Another conservative model was proposed by Rao and Shapiro (1970), who used the spectrum of the time series to detect changes in structure. Successive spectra of overlapping portions of the series are computed, with $\alpha$ determined as a function of the maximum change in the various frequency components. This procedure should make the forecasts highly resistant to outlying data points. Finally, the Kalman filter has been used to control the simple smoothing model (Bunn, 1981; Enns et al., 1982). Bunn gives a simple recursive approximation to the variances whereas Enns et al. give a maximum likelihood algorithm. Nembhard and Kao (2003), carried out a comparative study of two competing procedures for adaptive monitoring framework and determined that smoothed error procedure with control limits based on 3-3.5 deviations is preferred over adaptive auto regressive process of order one. 


\section{Chapter 3}

\section{Research methodology}

Having stated the scope of this research and reviewed some background and several related topics, the following methodologies (Sections 3.2 and 3.3) were used to achieve the specific objectives outlined in Chapter 1.

\subsection{Symbols and notations}

The symbols and notations that were employed in the research are summarized below:

$\begin{array}{ll}x_{t} & \text { Demand at time } \mathrm{t} \\ S_{t} & \text { Smoothed statistic at the end of time t (Forecast for next period) } \\ R_{a} & \text { Forecast smoothing parameter } \\ e_{t} & \text { Error } \\ M A D_{t} & \text { Mean Absolute Deviation at time } \mathrm{t} \\ R_{m} & \text { Sum of all previous errors at time } \mathrm{t} \\ S U M_{t} & \text { Cumulative sum tracking signal } \\ C U S U M_{t} & \text { Smoothed error at time t } \\ E_{t} & \text { Error smoothing parameter } \\ R_{e} & \text { Smoothed Error tracking signal } \\ S E T S_{t} & \text { Covariance at time t (smoothed) } \\ C O V_{t} & \text { Mean squared error at time t (smoothed) } \\ R_{c} & \text { Covariance smoothing Parameter } \\ M S E_{t} & \text { Cumeter }\end{array}$




$\begin{array}{ll}R_{m s} & \text { MSE smoothing Parameter } \\ R T S_{t} & \text { Autocorrelation tracking signal } \\ L_{i} & \text { Limit (+ or }- \text { ) on the } \text { ith backward CUSUM } \\ \sigma & \text { Standard deviation of forecast errors } \\ w \text { and } h & \text { Constants selected by simulation } \\ D_{t}^{+} & \text {Maximum difference at time t } \\ D_{t}^{-} & \text {Minimum difference at time t } \\ S U M n & \text { Sum of errors of n periods } \\ n & \text { Number of periods } \\ P C U S U M_{j} & \text { Parabolic mask tracking signal } \\ z^{*} & \text { Control limit for the parabolic mask tracking signal } \\ A R L 1 & \text { Average time between false alarm trips } \\ & \text { Average time until a false alarm trip occurs, starting from an }\end{array}$

\subsection{Determination of the Control Limits for tracking signals}

The procedure followed to determine the control limits to be used in the later part of the research is described below.

\subsubsection{Demand and Forecast generation}

Demands were simulated as normally distributed random variables with a mean of 1,000 and a standard deviation of 100 , truncated to integers. The demand (set of random numbers) was generated using MATLAB. The random number generator used was the 'RANDN' function, a standard multiplicative congruential generator. This generator has 
given excellent results on benchmark tests for independence and uniformity of random numbers (Gardner, 1983).

Once the demands were generated, next step was to generate the forecasts. The forecasts were generated using the simple exponential smoothing model.

$$
S_{T}=R_{a} * x_{T}+\left(1-R_{a}\right) * S_{T-1}
$$

The initial value of $S_{0}$ was set equal to the expected value of 1000 .

\subsubsection{Control limits for CUSUM tracking signal}

Once the forecasts were generated, the next step was to compute error, $S U M_{t}$, $M A D_{t}$ and the CUSUM ${ }_{t}$ values. The initial value of the MAD was set equal to the expected value of 80 . The forecast error, $e_{t}$, was computed as the actual time series value, $X_{t}$, minus the forecast $F_{t}$. SUMt, MADt and the CUSUMt were calculated using the following equations (also, see Equations 2.13, 2.14 and 2.15).

$$
\begin{aligned}
& S U M_{t}=e_{t}+S U M_{t-1} \\
& M A D_{t}=R_{m}\left|e_{t}\right|+\left(1-R_{m}\right) M A D_{t-1} \\
& \text { CUSUM }_{t}=\left|S U M_{t} / M A D_{t}\right|
\end{aligned}
$$

Control limits were determined based on simulation. The control limits were determined for $A R L 1$ of 25, 50 and 100 for false trips. Whenever the tracking signal exceeded the control limit, the sum of errors was reset to zero and the time since the last reset was recorded. However, the forecasts were not reset after a trip, since the false alarms are being simulated. To determine the appropriate control limits, the number of simulated demands was sufficient to generate approximately 1000 false alarms, which requires a series of about 100,000 demands when $A R L 1$ has a pre-specified limit of 100 
and so on. The average time between false alarm trips, ARL1, was computed as the number of demands generated divided by the number of false trips (number of times the CUSUM tracking signal has exceeded the control limits). The procedure to find control limits for simple CUSUM when $A R L 1=100$ is shown in Figure 3.1.

\subsubsection{Control limits for Smoothed Error Tracking Signal (SETS)}

The next step was to compute $E_{t}, M A D_{t}$ and $S E T S_{t}$ values. The initial values of $E_{0}$ and MAD are set equal to zero and the expected value (80) respectively. The updating equations are as follows (also, see Equations 2.20, 2.21 and 2.22).

$$
\begin{aligned}
& E_{t}=R_{e} e_{t}+\left(1-R_{e}\right) E_{t-1} \\
& M A D_{t}=R_{m}\left|e_{t}\right|+\left(1-R_{m}\right) M A D_{t-1} \\
& \operatorname{SETS}_{t}=\left|E_{t} / M A D_{t}\right|
\end{aligned}
$$

The control limits for SETS is found in a similar way to that of the simple CUSUM tracking signal. Whenever SETS exceeded the control limit, the smoothed error was reset to zero and the time since the last reset was recorded. However, the forecasts were not reset after a trip, since the false alarms are being simulated. The procedure to find control limits for $S E T S$ when $A R L 1=50$ is shown in Figure 3.2.

\subsubsection{Control limits for Autocorrelation Tracking Signal (RTS)}

The next step was to compute $C O V_{t}, M S E_{t}$ and $R T S_{t}$ values. The initial values of $C O V$ and $M S E$ are set equal to zero and expected value (100) respectively. The updating

equations for Autocorrelation tracking signal is as follows (also, see Equations 2.26, 2.27 and 2.28).

$$
C O V_{t}=e_{t} e_{t-1}+\left(1-R_{c}\right) C O V_{t-1}
$$




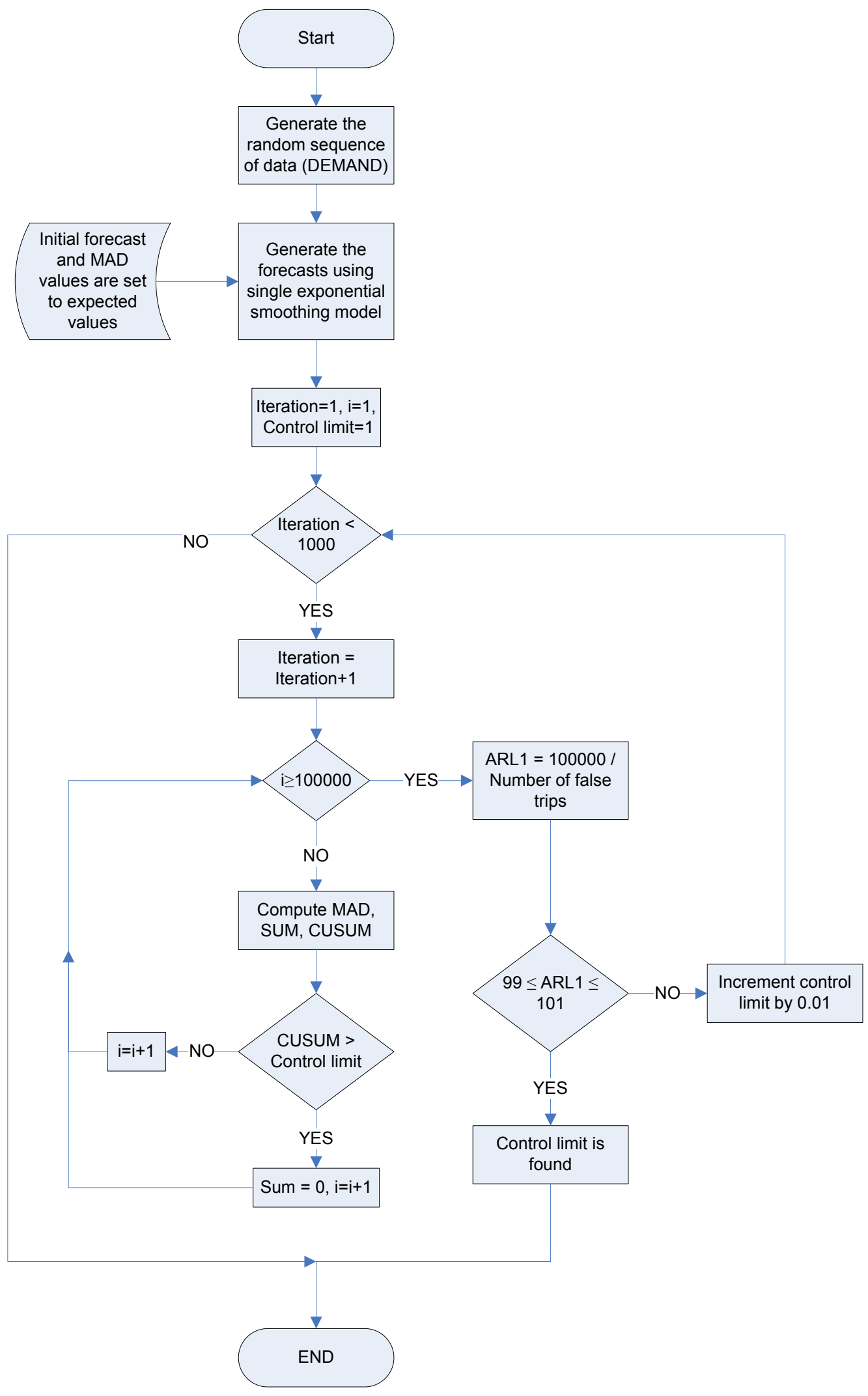

Figure 3.1 Procedure to find control limits for simple $C U S U M(A R L 1=100)$ 


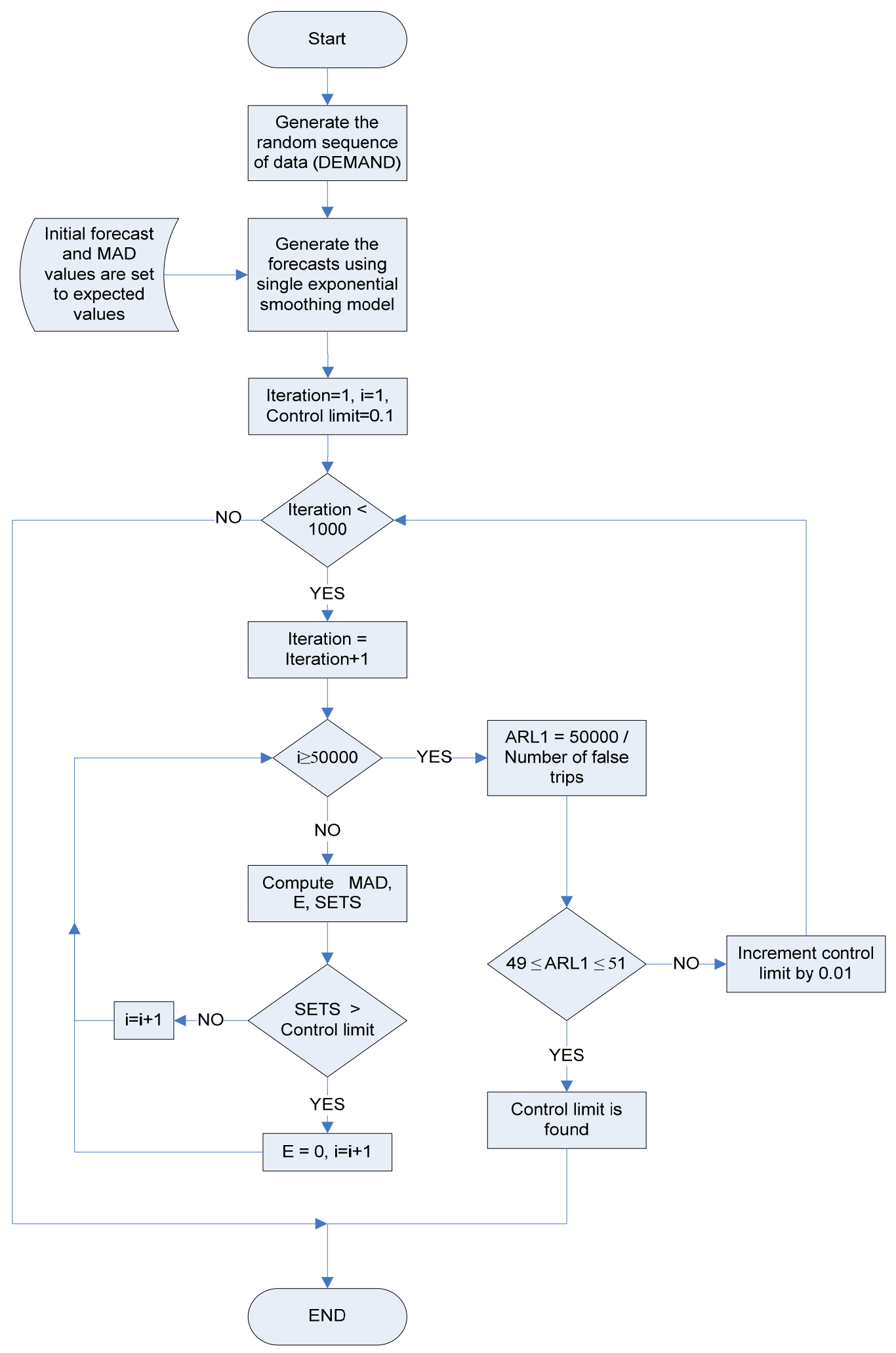

Figure 3.2 Procedure to find control limits for $\operatorname{SETS}(A R L 1=50)$ 


$$
\begin{aligned}
& M S E_{t}=e_{t-1}^{2}+\left(1-R_{m s}\right) M S E_{t-1} \\
& R T S_{t}=\left|C O V_{t} / M S E_{t}\right|
\end{aligned}
$$

The control limits for RTS was found in a similar way to that of the simple CUSUM tracking signal and SETS. Whenever RTS exceeded the control limit, the $C O V$ was reset to zero and the time since the last reset was recorded. However, the forecasts were not reset after a trip, since the false alarms are being simulated. The procedure to find control limits for $R T S$ when $A R L 1=25$ is shown in Figure 3.3.

\subsubsection{Control limits for Backward CUSUM tracking signal (BCUSUM)}

In this research a six period backward CUSUM control system was used. Control limits are setup in linear form, as

$$
L_{i}=\sigma w(i+h)
$$

Where $L_{i}$ is the limit (+ or -) on the $i$ th backward CUSUM, $\sigma$ is the standard deviation of forecast errors computed during a period when the system is in control, $w$ and $h$ are constants. The parameter used to check for positive bias is the minimum of the differences between the positive control limits and their corresponding CUSUMs, $L_{i}-S_{i}$, from the last period. The test for positive bias is

$$
D_{t}^{+}=\operatorname{MIN}\left[D_{t-1}^{+}, L_{0}\right]+\sigma w-e_{t}
$$

$D_{0}^{+}$is set equal to $L_{0}$. If $D_{t}^{+}<0$, lack of control is signaled. Also, see Equation (2.18).

The other parameter used to check for negative bias, is the maximum of the differences between the negative control limits and their CUSUMs is $-L_{i}-S_{i}$. The test for negative bias is

$$
D_{t}^{-}=M A X\left[D_{t-1}^{-},-L_{0}\right]-\sigma w-e_{t}
$$


$D_{0}^{-}$is set equal to $-L_{0}$. If $D_{t}^{-}>0$, lack of control is signaled. Also, see Equation (2.19).

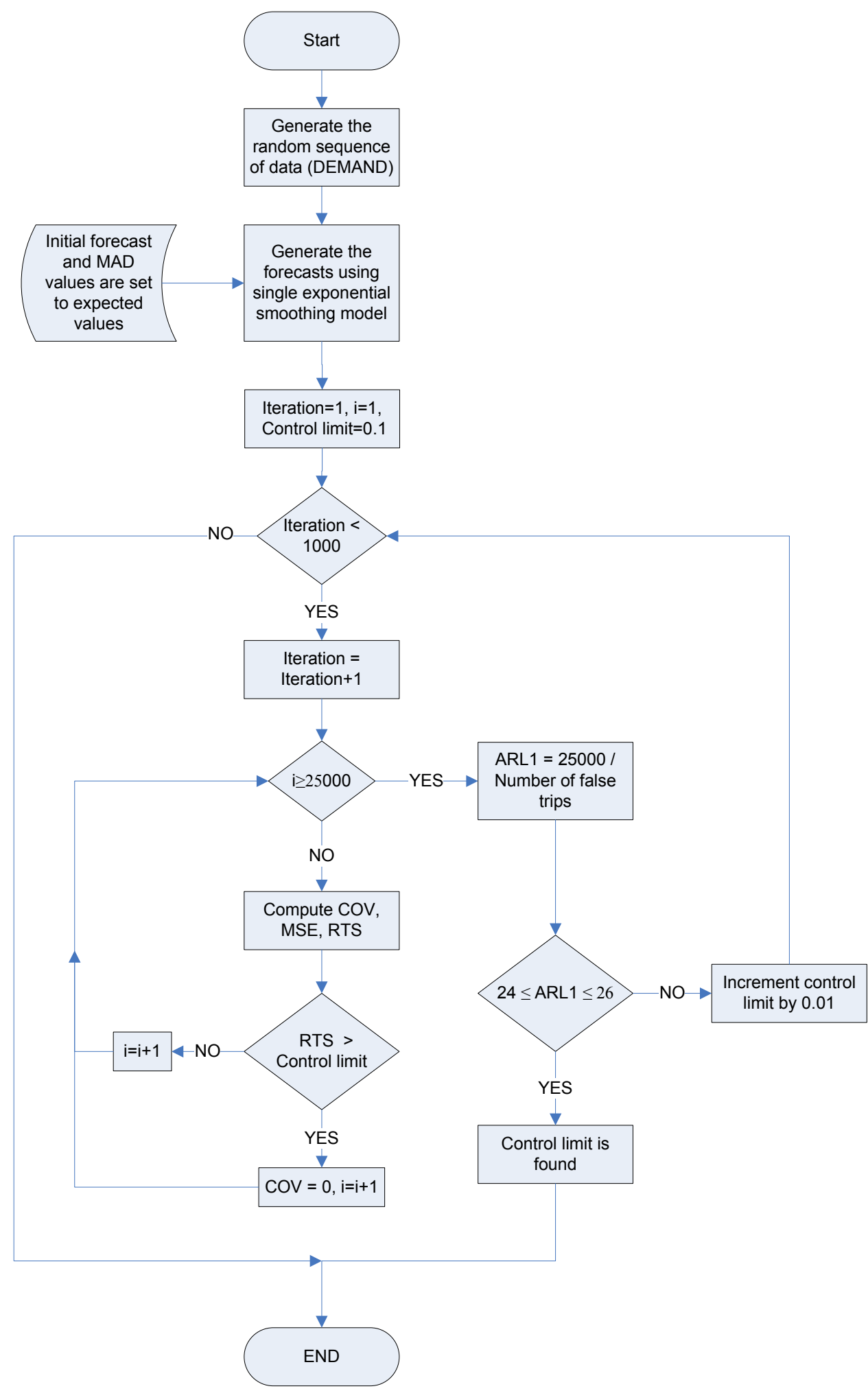

Figure 3.3 Procedure to find control limits for $R T S(A R L 1=25)$ 
The only published control limits for forecast errors that are based on a large sample size, with a run in period to wash out initial conditions, are those given by Golder and Settle (1976) and Gardner (1983).

Table 3.1 BCUSUM response comparisons, $R_{a}=0.1$ and $A R L 1=50$

\begin{tabular}{|c|c|c|}
\hline \multirow{2}{*}{$\begin{array}{c}\text { Control limits } \\
(\mathrm{w}, \mathrm{h})\end{array}$} & \multicolumn{2}{|c|}{ ARL to detect bias } \\
\cline { 2 - 3 } & $1.5 \sigma$ & $3.0 \sigma$ \\
\hline $0.1,52.4$ & 3.9 & 1.9 \\
$0.2,21.2$ & 3.7 & 1.8 \\
$0.3,11.7$ & 3.7 & 1.7 \\
$0.4,7.6$ & 4.0 & 1.6 \\
\hline
\end{tabular}

Table 3.1 illustrates the search process used to find control limits in this research. The process used to find the control limits matches with the one used by Gardner, the difference being Gardner used $A R L 2$ and in this research $A R L 1$ was used. The $w$ parameter was varied by increments of 0.1 , starting at 0.1 , since Golder and Settle's and Gardner's control limits indicate that the best w values are usually fractional. At each value of $w$, the $h$ parameter was varied to find the limits yielding an $A R L 1=50$ periods. Whenever $D_{t}^{+}<0, D_{t}^{+}$was set equal to $L_{0}$ and, $D_{t}^{-}>0, D_{t}^{-}$was set equal to $-L_{0}$. The time since the last reset was recorded. To determine the appropriate control limits, the number of simulated demands was sufficient to generate approximately 1000 false alarms, which requires a series of about 100,000 demands when $A R L 1$ has a pre-specified limit of 100 and so on. The procedure to find control limits for BCUSUM when $A R L 1=50$ is shown in Figure 3.4. Using those $w$ and $h$ values, the $A R L s$ to detect $1.5 \sigma$ and $3.0 \sigma$ step changes in the mean were measured. This procedure was continued until the $A R L 1$ at $1.5 \sigma$ passed through a minimum. If there was a tie for the best limits at $1.5 \sigma$, the tie was broken with 


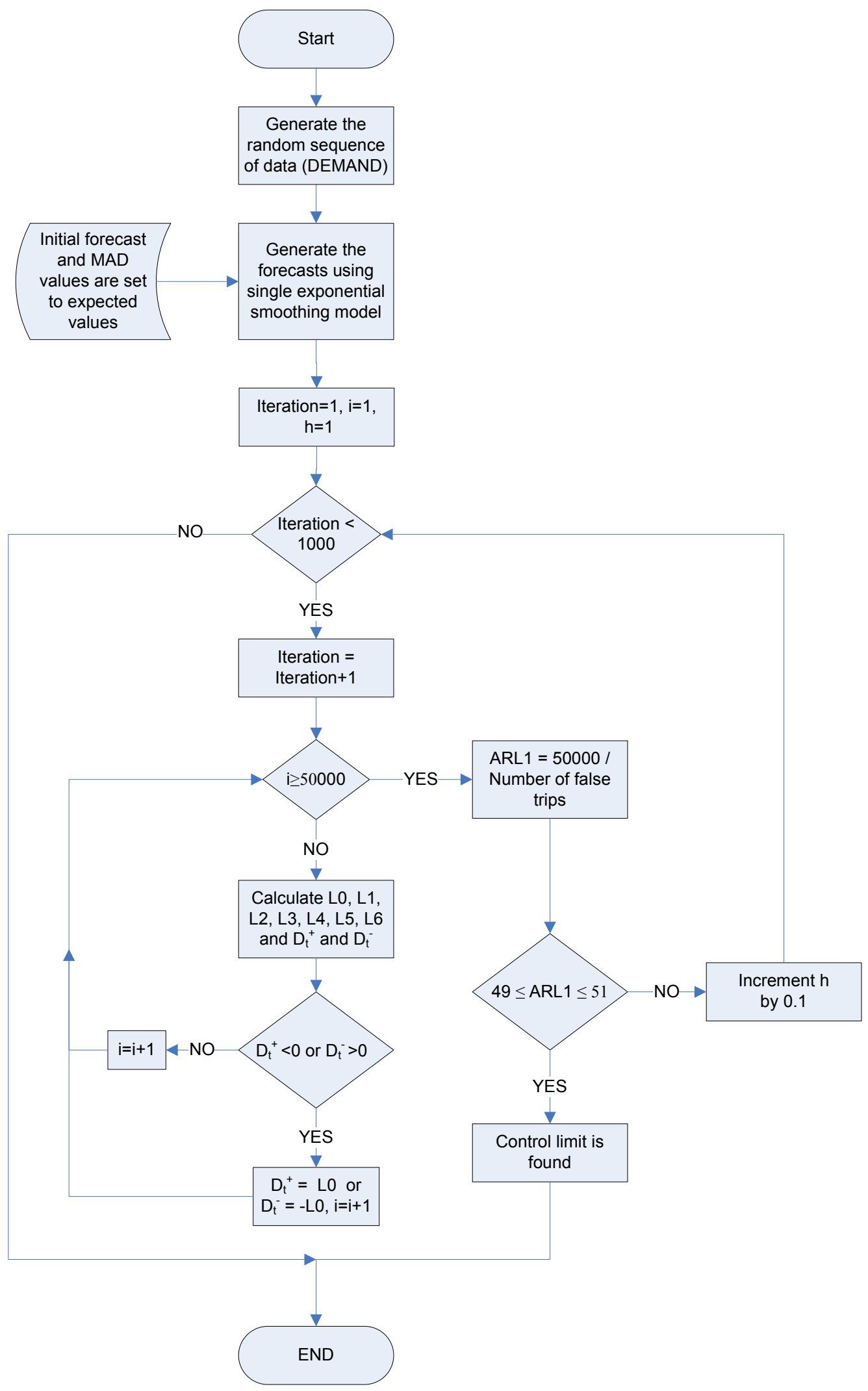

Figure 3.4 Procedure to find control limits for $B C U S U M(A R L 1=50, w=0.1)$ 
the minimum ARL1 at $3.0 \sigma$. Given the best $w$ value using these criteria, $h$ was varied to find control limits for $A R L 1=25$ and 100 periods on unbiased errors. Although choosing the control limits in this fashion is somewhat arbitrary, it seemed to be the best way to deal with the complexities of the response rates. Bias equal to $1.5 \sigma$ is about the smallest level that any tracking signal can detect in a reasonable number of periods. Since the $A R L 1$ at $3.0 \sigma$ was always two periods or less for virtually any set of limits, it seemed best to minimize the $A R L 1$ at $1.5 \sigma$ rather than $3.0 \sigma$.

\subsubsection{Control limits for Parabolic CUSUM Tracking Signal (PCUSUM)}

In this research a twelve period parabolic CUSUM control system was used. The next step was to compute $\mathrm{CUSUM}_{j}$ and $\mathrm{CUSUM}_{n}$ using the following equations.

$$
\operatorname{CUSUM}_{j}=\sum_{i=1}^{j} x_{i}, j=1,2, \ldots
$$

CUSUM $_{j}$, in Equation (3.14) can be written as

$$
\begin{aligned}
\operatorname{CUSUM}_{j} & =\sum_{i=1}^{j} x_{i} \\
& =\sum_{i=1}^{n} x_{i}-\sum_{i=j+1}^{n} x_{i} \quad 1 \leq j \leq n \\
& =\text { CUSUM } \\
n & -\sum_{i=j+1}^{n} x_{i}
\end{aligned}
$$

The following parabolic limits that should be plotted against CUSUMj $(1<t<\mathrm{n}-1)$ :

$$
\begin{aligned}
& \operatorname{PCUSUM}_{j} \geq z^{*} \sigma \sqrt{(n-j)}+\operatorname{CUSUM}_{n} \\
& \operatorname{PCUSUM}_{j} \leq-z * \sigma \sqrt{(n-j)}+\text { CUSUM }_{n}
\end{aligned}
$$


The $\mathrm{z}^{*}$ in Equation (3.16) is the control limits which was chosen such that the CUSUM achieves a pre-specified in-control ARL of 25, 50 and 100. In using Equation (3.16), the initial value of CUSUM is set equal to zero. The procedure to find control limits $\left(\mathrm{z}^{*}\right)$ for PCUSUM when $A R L 1=50$ is shown in Figure 3.5. Also, see Equations (2.29) and (2.30).

\subsection{Determination of bias in the tracking signals}

All tracking signals were compared at control limits yielding ARLs of 25, 50 and 100 periods on unbiased errors, from simple exponential smoothing. The procedure to determine bias for the five different tracking signals is described below.

\subsubsection{Demand and Forecast generation}

The demand (set of random numbers) was generated using MATLAB. The random number generator used was the ' $R A N D N$ ' function. Demands were simulated as normally distributed random variables with a mean of 1000 and a standard deviation of 100 , truncated to integers. For simulating response to a step, 1000 series of demands were generated, each covering enough periods to allow a run-in (described below) and 75 periods after the step. To minimize the effects of initial values, a run-in period was used in which no data were collected. The length of the run-in period was 40 periods. After the run-in period, a step increase in the mean level was added to each series, and the Average Run Length (ARL 1) to detect the step was measured. A step size of $1.0 \sigma$ was introduced in every run. The maximum bias used in this research was $3.0 \sigma$.

In the next step, the forecasts were generated using the simple exponential smoothing model (3.1). The initial forecast was set equal to expected value of 1000 . 


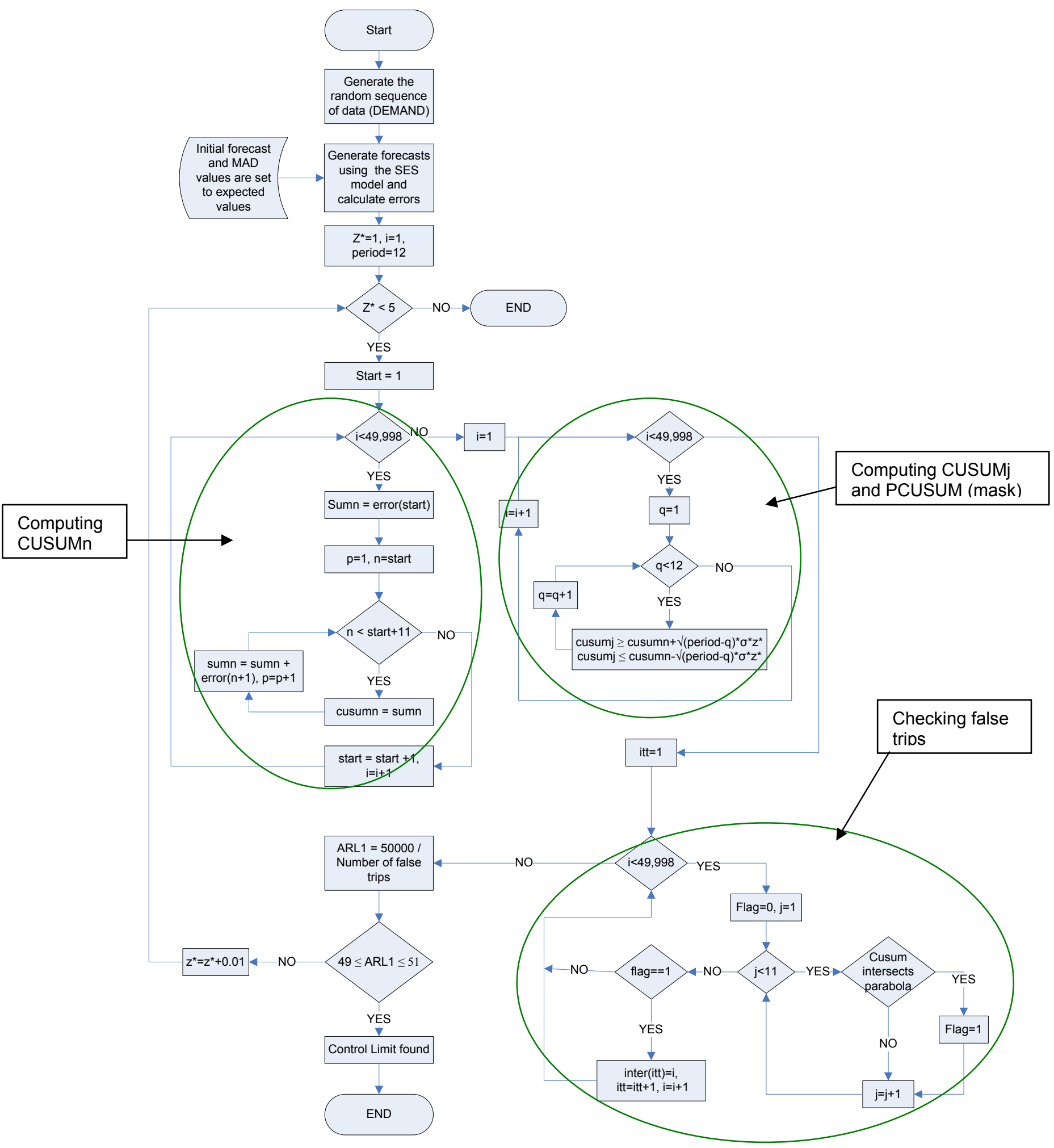

Figure 3.5 Procedure to find control limits for PCUSUM $(A R L 1=50)$ 


\subsubsection{CUSUM tracking signal}

Once the forecasts were generated, the next step was to compute error, SUMt, $M A D t$ and the CUSUMt values. The initial value of the $M A D$ was set equal to the

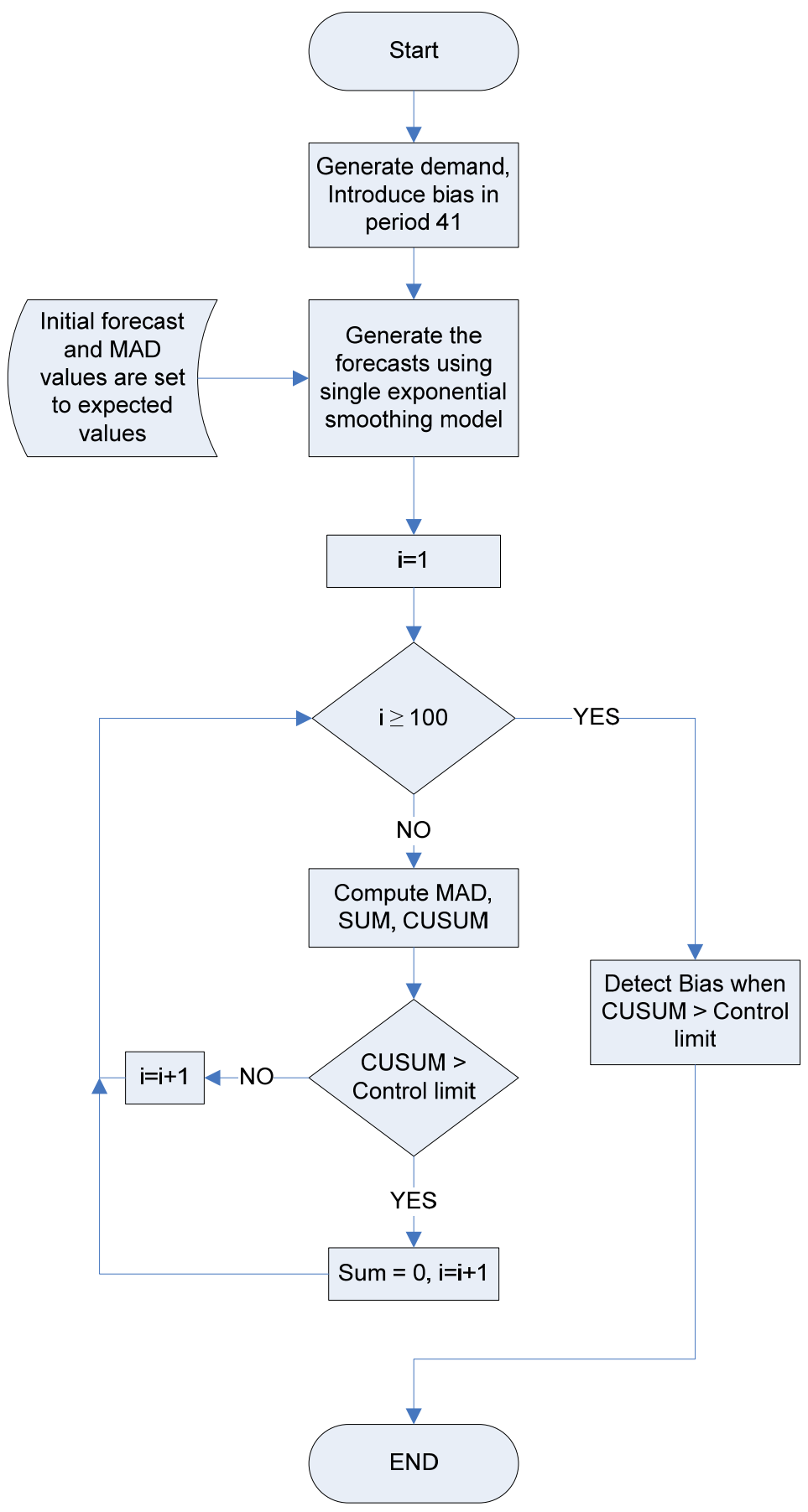

Figure 3.6 Procedure to determine bias for CUSUM tracking signal. 
expected value of 80 . The forecast error, $e_{t}$, was computed as the actual time series value, $x_{t}$, minus the forecast $F_{t} . S U M t$, MADt and the CUSUMt were calculated using Equations (3.2), (3.3) and (3.4) respectively. The corresponding control limits which were determined earlier yielding $A R L s$ of 25, 50 and 100 periods on unbiased errors were used to determine the bias. Bias is found when ratio of SUM/MAD exceeds the control limit. Whenever the tracking signal exceeded the control limit, the sum of errors was reset to zero and the time since the last reset was recorded. A flow chart describing the procedure to find bias in the error for simple CUSUM tracking signal is shown in Figure 3.6.

\subsubsection{Smoothed error tracking signal (SETS)}

The values of $E_{t}, M A D_{t}$ and $S E T S_{t}$ were computed using Equations (3.5), (3.6) and (3.7) respectively. The initial values of $\mathrm{E}$ and $\mathrm{MAD}$ are set equal to zero and the expected value (80) respectively. The corresponding control limits which were determined earlier yielding $A R L s$ of 25, 50 and 100 periods on unbiased errors were used to determine the bias. Bias is found when ratio of $E / M A D$ exceeds the control limit. Whenever the tracking signal exceeded the control limit, the sum of errors was reset to zero and the time since the last reset was recorded. A flow chart describing the procedure to find bias in the error for smoothed error tracking signal is shown in Figure 3.7.

\subsubsection{Auto-correlation tracking signal (RTS)}

The values of $C O V_{t}, M S E_{t}$ and $R T S_{t}$ are computed using Equations (3.8), (3.9) and (3.10) respectively. The initial values of $C O V$ and $M S E$ are set equal to zero and expected value (100) respectively. The corresponding control limits which were determined earlier yielding $A R L s$ of 25, 50 and 100 periods on unbiased errors were used to determine the 
bias. Bias is found when ratio of COV/MSE exceeds the control limit. Whenever the tracking signal exceeded the control limit, the covariance was reset to zero and the time

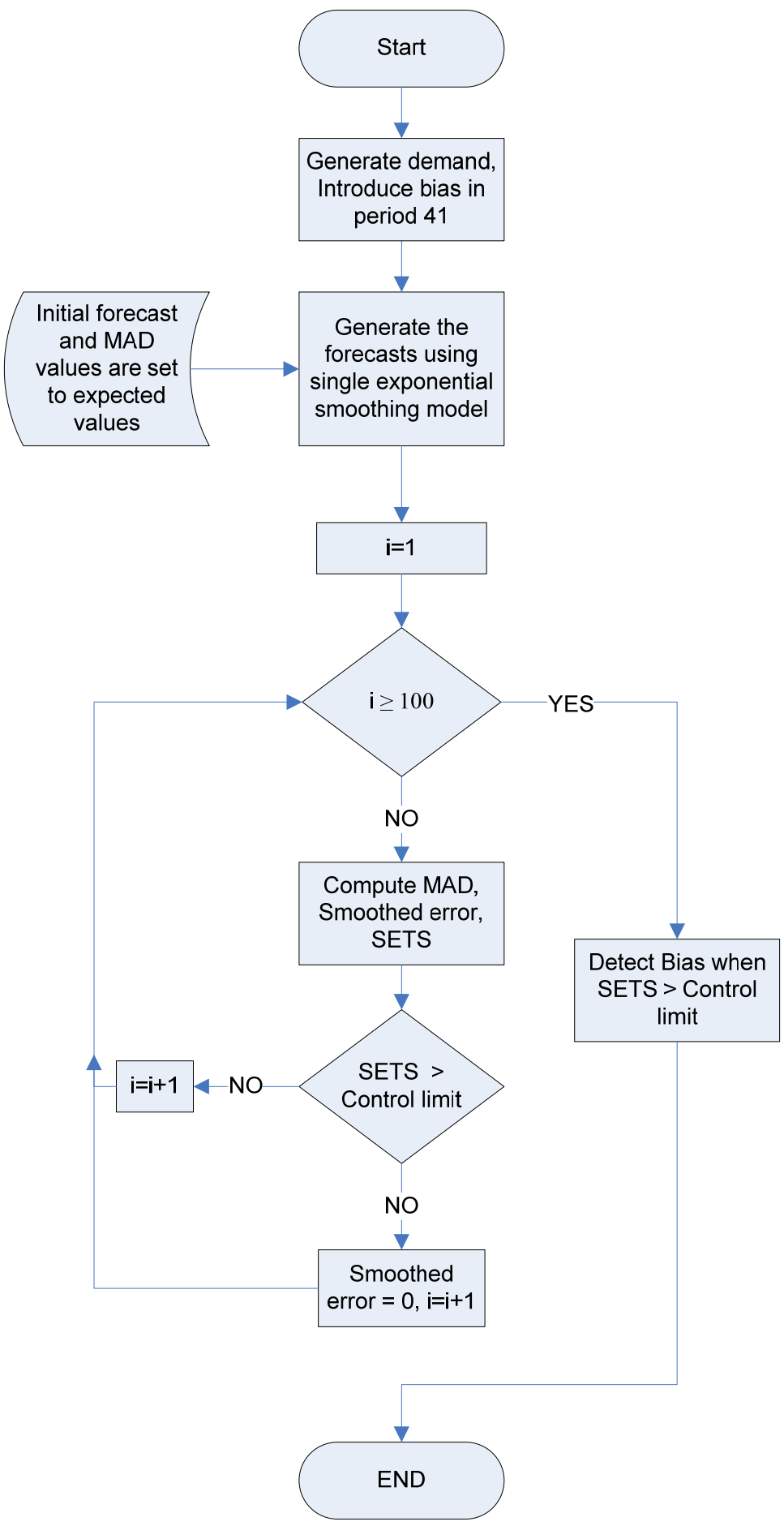

Figure 3.7 Procedure to determine bias for smoothed error tracking signal. 


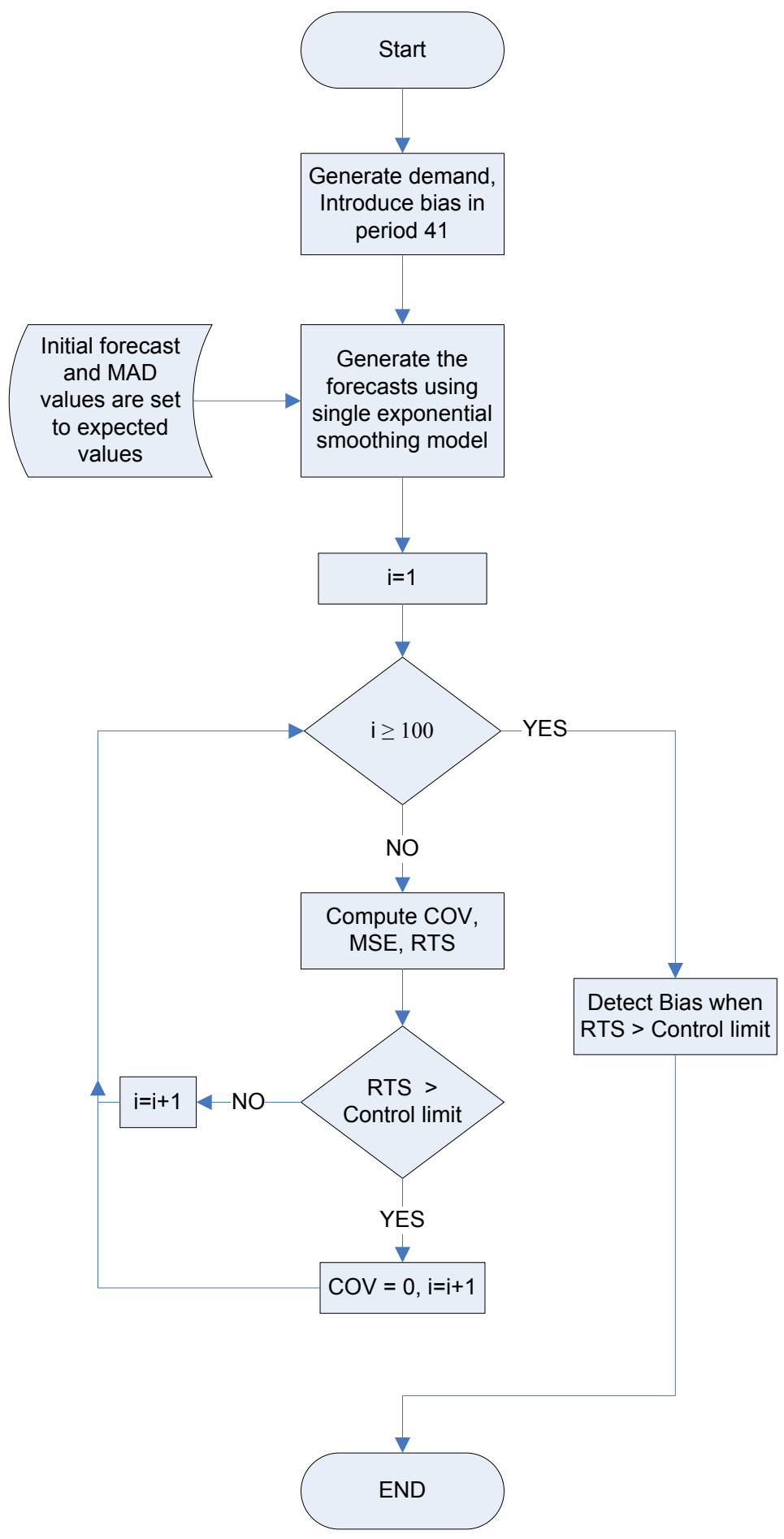

Figure 3.8 Procedure to determine bias for Auto-correlation tracking signal. 
since the last reset was recorded. Average Run Length (ARL 1) to detect the step was measured. A flow chart describing the procedure to find bias in the error for Autocorrelation tracking signal is shown in Figure 3.8.

\subsubsection{Backward CUSUM tracking signal (BCUSUM)}

A six period backward CUSUM control system was used in this research. The control limits were set up in the linear form as described earlier in section 3.2.5 (Equation 3.11). The tests for positive bias and negative bias were carried out as described in Equations (3.12) and (3.13). The corresponding control limits ( $w$ and $h)$ which were determined earlier yielding $A R L s$ of 25, 50 and 100 periods on unbiased errors were used to determine the bias. Whenever $D_{t}^{+}<0, D_{t}^{+}$was set equal to $L_{0}$ and, $D_{t}^{-}>0, D_{t}^{-}$was set equal to $-L_{0}$. The time since the last reset was recorded. The Average Run Length (ARL1) to detect the step was measured. A flow chart describing the procedure to find bias in the error for the Backward CUSUM tracking signal is shown in Figure 3.9.

\subsubsection{Parabolic CUSUM tracking signal (PCUSUM)}

A twelve period parabolic CUSUM control system was used in this research. The values of CUSUMn and CUSUMj are computed using Equations (3.14) and (3.15) respectively. The parabolic limits that should be plotted against CUSUMj is computed using Equation (3.16). In using Equation (3.16), the initial value of CUSUM is set equal to zero. The corresponding control limits $\left(z^{*}\right)$ which were determined earlier yielding $A R L s$ of 25, 50 and 100 periods on unbiased errors were used to determine the bias. To test for bias, a parabola was constructed over the most recent CUSUM. The arms of the parabola point backward in time. If all preceding sums stay within the arms of the 
parabola, the system is in control. If any previous CUSUM falls outside the area of the parabola, the system is out of control. A flow chart describing the procedure to find bias in the error for the parabolic CUSUM tracking signal is shown in Figure 3.10.

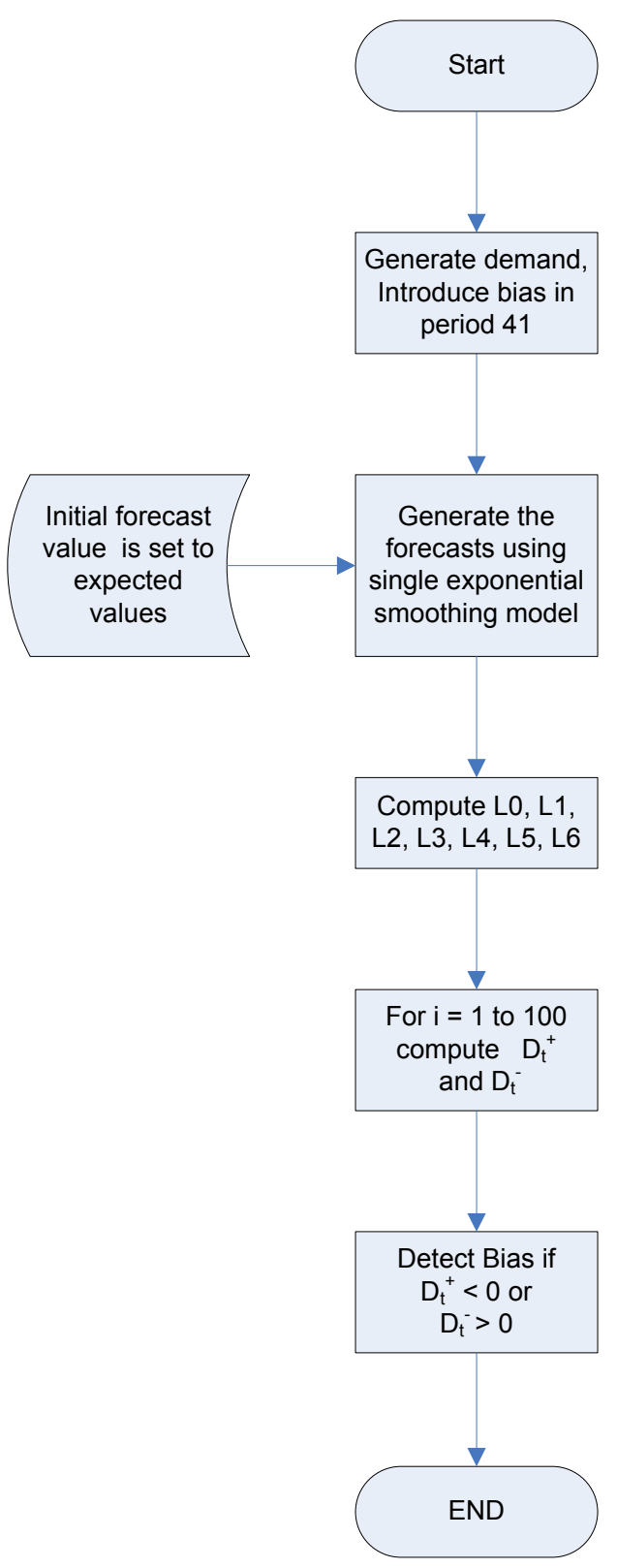

Figure 3.9 Procedure to determine bias for Backward CUSUM tracking signal. 


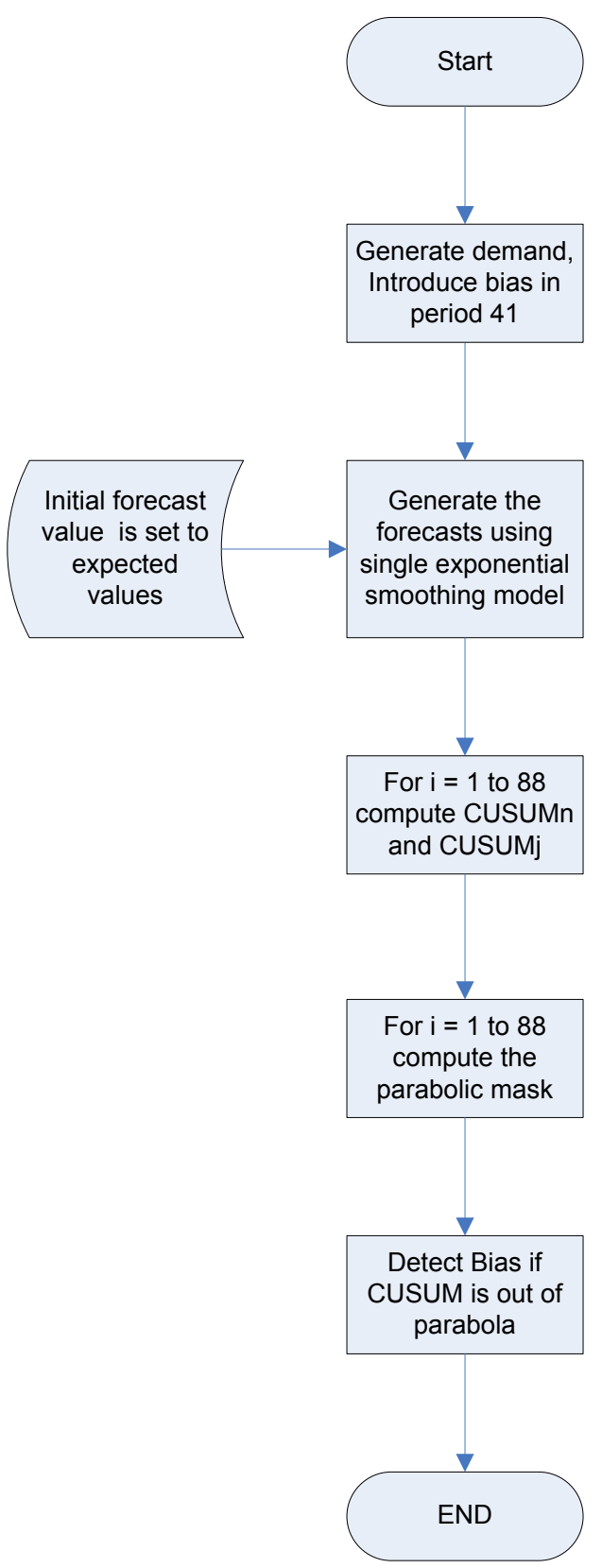

Figure 3.10 Procedure to determine bias for Parabolic CUSUM tracking signal. 


\section{Chapter 4}

\section{Analysis of Results}

\subsection{Introduction}

The control limits were determined based on the set criteria as explained in chapter 3. After that, the tracking signals CUSUM, SETS, RTS, BCUSUM and PCUSUM were compared according to the criterion $A R L 1$. All tracking signals were compared at control limits yielding $A R L s$ of 25, 50 and 100 periods on unbiased errors, from simple exponential smoothing. The tracking signals were compared for different smoothing constants, for step responses of $1 \sigma, 2 \sigma$, and $3 \sigma$ and also for a random step increase. The results obtained are discussed in the following sections.

\subsection{Control Limits}

Tables 4.1 to 4.9 present the control limits based on the $A R L 1$ criterion. The forecasting model used is single exponential smoothing. The control limits were obtained by a search routine (see section 3.2). The control limits were determined for smoothing constants $0.05,0.1,0.15$ and 0.2 .

Table 4.1 CUSUM control limits for $\boldsymbol{R}_{a}=\boldsymbol{R}_{m}$

\begin{tabular}{|c|c|c|c|c|}
\hline \multirow[b]{2}{*}{ ARL1 } & \multicolumn{4}{|c|}{$\mathbf{R a}$} \\
\hline & 0.05 & 0.1 & 0.15 & 0.2 \\
\hline 25 & 5.00 & 4.72 & $\overline{4.40}$ & 4.26 \\
\hline 50 & 6.75 & 6.32 & 5.80 & 5.54 \\
\hline 100 & 8.99 & 7.86 & 6.95 & 6.60 \\
\hline
\end{tabular}


Table 4.2 CUSUM Control Limits for $\boldsymbol{R}_{m}=\mathbf{0 . 0 5}$

\begin{tabular}{|c|c|c|c|c|}
\hline \multirow{2}{*}{ ARL1 } & \multicolumn{4}{|c|}{$\mathbf{R a}$} \\
\cline { 2 - 5 } & $\mathbf{0 . 0 5}$ & $\mathbf{0 . 1}$ & $\mathbf{0 . 1 5}$ & $\mathbf{0 . 2}$ \\
\hline \hline $\mathbf{2 5}$ & 5.00 & 4.61 & 4.33 & 4.29 \\
\hline $\mathbf{5 0}$ & 6.75 & 6.20 & 5.66 & 5.23 \\
$\mathbf{1 0 0}$ & 8.99 & 7.56 & 6.83 & 6.30 \\
\hline
\end{tabular}

Table 4.1 shows the control limits for CUSUM when $R_{m}$, the smoothing parameter for $M A D$, is set equal to the forecast's parameter (i.e., $R_{m}=R_{a}$ ). Table 4.2 is similar except that $R_{m}=0.05$. Table 4.3 shows the control limits for SETS when $R_{m}=R_{e}$ and Table 4.4 when $R_{m}=0.05$. Control limits for the RTS are shown in Table 4.5 for $R_{m s}=R_{c}$. and Table 4.6 for $R_{m s}=0.05$.

Table 4.3 SETS Control Limits for $R_{m}=R_{e}$.

\begin{tabular}{|c|c|c|c|c|c|}
\hline \multirow[b]{2}{*}{ ARL1 } & \multirow[b]{2}{*}{$\operatorname{Re}$} & \multicolumn{4}{|c|}{$\mathbf{R a}$} \\
\hline & & 0.05 & 0.1 & 0.15 & 0.2 \\
\hline \multirow{4}{*}{25} & 0.05 & $\overline{0.214}$ & $\overline{c 0.201}$ & $\overline{0.192}$ & $\begin{array}{c}0.184 \\
\end{array}$ \\
\hline & 0.1 & 0.379 & 0.359 & 0.348 & 0.335 \\
\hline & 0.15 & 0.503 & 0.493 & 0.476 & 0.461 \\
\hline & 0.2 & 0.631 & 0.604 & 0.585 & 0.568 \\
\hline \multirow{4}{*}{50} & 0.05 & 0.268 & 0.247 & 0.232 & 0.216 \\
\hline & 0.1 & 0.452 & 0.428 & 0.404 & 0.382 \\
\hline & 0.15 & 0.592 & 0.567 & 0.541 & 0.518 \\
\hline & 0.2 & 0.699 & 0.679 & 0.656 & 0.634 \\
\hline \multirow{4}{*}{100} & 0.05 & 0.317 & 0.281 & 0.258 & 0.240 \\
\hline & 0.1 & 0.514 & 0.480 & 0.449 & 0.422 \\
\hline & 0.15 & 0.660 & 0.628 & 0.591 & 0.567 \\
\hline & 0.2 & 0.767 & 0.733 & 0.711 & 0.682 \\
\hline
\end{tabular}


Table 4.4 SETS Control Limits for $\boldsymbol{R}_{\boldsymbol{m}}=\mathbf{0 . 0 5}$

\begin{tabular}{|c|c|c|c|c|c|}
\hline \multirow{3}{*}{ ARL1 } & Re & \multicolumn{4}{|c|}{$\mathbf{R a}$} \\
\cline { 3 - 6 } $\mathbf{5} 5$ & $\mathbf{0 . 0 5}$ & 0.214 & 0.201 & 0.192 & 0.184 \\
\cline { 2 - 6 } & $\mathbf{0 . 1}$ & 0.379 & 0.371 & 0.352 & 0.338 \\
& $\mathbf{0 . 1 5}$ & 0.532 & 0.503 & 0.492 & 0.472 \\
& $\mathbf{0 . 2}$ & 0.663 & 0.641 & 0.620 & 0.604 \\
\hline \multirow{5}{*}{$\mathbf{5 0}$} & $\mathbf{0 . 0 5}$ & 0.268 & 0.247 & 0.232 & 0.216 \\
\cline { 2 - 6 } & $\mathbf{0 . 1}$ & 0.467 & 0.433 & 0.410 & 0.394 \\
\cline { 2 - 6 } & $\mathbf{0 . 1 5}$ & 0.626 & 0.598 & 0.568 & 0.547 \\
& $\mathbf{0 . 2}$ & 0.777 & 0.737 & 0.716 & 0.689 \\
\hline \multirow{5}{*}{$\mathbf{1 0 0}$} & $\mathbf{0 . 0 5}$ & 0.317 & 0.281 & 0.258 & 0.240 \\
& $\mathbf{0 . 1}$ & 0.530 & 0.491 & 0.456 & 0.432 \\
\cline { 2 - 6 } & $\mathbf{0 . 1 5}$ & 0.707 & 0.669 & 0.636 & 0.600 \\
& $\mathbf{0 . 2}$ & 0.868 & 0.832 & 0.790 & 0.765 \\
\hline
\end{tabular}

Table 4.5 RTS Control Limits for $\boldsymbol{R}_{m s}=\boldsymbol{R}_{c}$.

\begin{tabular}{|c|c|c|c|c|c|}
\hline \multirow{3}{*}{ ARL1 } & \multirow{R}{*}{$\mathbf{R c}$} & \multicolumn{4}{|c|}{$\mathbf{R a}$} \\
\cline { 3 - 6 } $\mathbf{2 5}$ & $\mathbf{0 . 0 5}$ & $\mathbf{0 . 1}$ & $\mathbf{0 . 1 5}$ & $\mathbf{0 . 2}$ \\
\hline \hline \multirow{5}{*}{$\mathbf{5 0 . 0 5}$} & 0.062 & 0.050 & 0.034 & 0.029 \\
\cline { 2 - 6 } & $\mathbf{0 . 1}$ & 0.153 & 0.133 & 0.114 & 0.106 \\
& $\mathbf{0 . 1 5}$ & 0.261 & 0.240 & 0.219 & 0.187 \\
\cline { 2 - 6 } & $\mathbf{0 . 2}$ & 0.345 & 0.337 & 0.308 & 0.277 \\
\hline \multirow{5}{*}{$\mathbf{0 . 0 5}$} & 0.121 & 0.100 & 0.083 & 0.065 \\
\cline { 2 - 6 } & $\mathbf{0 . 1}$ & 0.262 & 0.243 & 0.205 & 0.189 \\
\cline { 2 - 6 } & $\mathbf{0 . 1 5}$ & 0.386 & 0.359 & 0.336 & 0.309 \\
& $\mathbf{0 . 2}$ & 0.505 & 0.480 & 0.449 & 0.417 \\
\hline \multirow{3}{*}{$\mathbf{0 . 0 5}$} & 0.183 & 0.163 & 0.137 & 0.116 \\
& $\mathbf{0 . 1}$ & 0.364 & 0.328 & 0.304 & 0.276 \\
\cline { 2 - 6 } & $\mathbf{0 . 1 5}$ & 0.497 & 0.479 & 0.448 & 0.413 \\
\cline { 2 - 6 } & $\mathbf{0 . 2}$ & 0.631 & 0.596 & 0.574 & 0.545 \\
\hline
\end{tabular}

Table 4.6 RTS Control Limits for $\boldsymbol{R}_{m s}=\mathbf{0 . 0 5}$

\begin{tabular}{|c|c|c|c|c|c|}
\hline \multirow{3}{*}{ ARL1 } & Rc & \multicolumn{4}{|c|}{$\mathbf{R a}$} \\
\cline { 3 - 6 } $\mathbf{5} \mathbf{2 5}$ & $\mathbf{0 . 0 5}$ & 0.062 & 0.050 & 0.034 & 0.029 \\
& $\mathbf{0 . 1}$ & 0.079 & 0.064 & 0.053 & 0.051 \\
& $\mathbf{0 . 1 5}$ & 0.084 & 0.077 & 0.064 & 0.060 \\
& $\mathbf{0 . 2}$ & 0.083 & 0.079 & 0.070 & 0.063 \\
\hline \multirow{5}{*}{$\mathbf{5 0}$} & $\mathbf{0 . 0 5}$ & 0.121 & 0.100 & 0.083 & 0.065 \\
& $\mathbf{0 . 1}$ & 0.128 & 0.110 & 0.104 & 0.093 \\
& $\mathbf{0 . 1 5}$ & 0.127 & 0.116 & 0.115 & 0.096 \\
& $\mathbf{0 . 2}$ & 0.127 & 0.116 & 0.106 & 0.100 \\
\hline \multirow{5}{*}{$\mathbf{1 0 0}$} & $\mathbf{0 . 0 5}$ & 0.183 & 0.163 & 0.137 & 0.116 \\
& $\mathbf{0 . 1}$ & 0.177 & 0.166 & 0.147 & 0.136 \\
& $\mathbf{0 . 1 5}$ & 0.170 & 0.163 & 0.147 & 0.136 \\
& $\mathbf{0 . 2}$ & 0.168 & 0.152 & 0.144 & 0.134 \\
\hline
\end{tabular}




\subsubsection{Choice of smoothing Parameters $\boldsymbol{R}_{m}$ and $\boldsymbol{R}_{m s}$}

The best choice of $R_{m} / R_{m s}$ was tested by simulating the response of CUSUM tracking signal to a step (permanent increase) in the average demand level. The control limits are chosen so that $A R L 1=100$. For the first test, the demand was permanently increased by 1.5 times its standard deviation. The results showed that the detection probability is higher with $R_{m}\left(R_{m s}\right)=0.05$. This was true for all values of the forecast smoothing parameter $R_{a}$. The experiment was repeated with a 3 sigma step under the same conditions with similar results. If there was a tie for the best limits at $1.5 \sigma$, the tie was broken with the minimum ARL at 3.0 0 . Additional experiments showed that the same general conclusions hold if $A R L 1$ is set at 25 or 50 rather than 100 or if the SETS or RTS is used instead of CUSUM. Hence $R_{m}\left(R_{m s}\right)=0.05$ is superior for commonly used values of $R_{a}$. Tables 4.7 and 4.8 SETS, Tables $4.9,4.10$ and 4.11 illustrates the process described above for CUSUM and RTS respectively. 
Table 4.7 ARL1 Comparison for different smoothing parameters $R_{a}$ at $1.5 \sigma$ (SETS)

\begin{tabular}{|c|c|c|c|c|c|c|c|c|c|c|c|c|c|}
\hline \multirow[b]{2}{*}{$\mathbf{R m}$} & \multirow[b]{2}{*}{ ARL1 } & \multirow[b]{2}{*}{$\mathbf{R e}$} & \multicolumn{4}{|c|}{$\mathbf{R a}$} & \multirow[b]{2}{*}{$\mathbf{R m}$} & \multirow[b]{2}{*}{ ARL1 } & \multirow[b]{2}{*}{$\operatorname{Re}$} & \multicolumn{4}{|c|}{$\mathbf{R a}$} \\
\hline & & & 0.05 & 0.1 & 0.15 & 0.2 & & & & 0.05 & 0.1 & 0.15 & 0.2 \\
\hline \multirow{12}{*}{0.05} & \multirow{4}{*}{25} & 0.05 & 3.46 & 3.50 & 3.58 & 3.62 & \multirow{12}{*}{0.15} & \multirow{4}{*}{25} & 0.05 & 4.29 & 4.17 & 4.34 & 4.27 \\
\hline & & 0.1 & 3.34 & 3.32 & 3.65 & 3.68 & & & 0.1 & 3.77 & 3.91 & 3.95 & 4.29 \\
\hline & & 0.15 & 3.16 & 3.16 & 2.77 & 3.85 & & & 0.15 & 3.53 & 3.70 & 3.94 & 4.38 \\
\hline & & 0.2 & 3.02 & 3.05 & 3.41 & 3.93 & & & 0.2 & 3.42 & 3.63 & 4.14 & 4.43 \\
\hline & \multirow{4}{*}{50} & 0.05 & 4.28 & 4.39 & 4.37 & 4.42 & & \multirow{4}{*}{50} & 0.05 & 5.76 & 5.63 & 5.80 & 5.71 \\
\hline & & 0.1 & 4.13 & 4.16 & 4.46 & 4.51 & & & 0.1 & 5.39 & 5.34 & 5.43 & 5.83 \\
\hline & & 0.15 & 4.02 & 3.79 & 3.52 & 4.66 & & & 0.15 & 5.27 & 5.41 & 5.51 & 5.86 \\
\hline & & 0.2 & 3.89 & 3.97 & 4.31 & 4.76 & & & 0.2 & 4.96 & 5.29 & 5.73 & 5.99 \\
\hline & \multirow{4}{*}{100} & 0.05 & 5.03 & 4.96 & 4.83 & 4.91 & & \multirow{4}{*}{100} & 0.05 & 6.96 & 6.74 & 6.57 & 6.38 \\
\hline & & 0.1 & 4.90 & 4.81 & 4.99 & 5.03 & & & 0.1 & 6.41 & 6.52 & 6.63 & 6.51 \\
\hline & & 0.15 & 4.67 & 4.48 & 4.17 & 5.17 & & & 0.15 & 6.38 & 6.44 & 6.59 & 6.67 \\
\hline & & 0.2 & 4.41 & 4.53 & 4.89 & 5.26 & & & 0.2 & 6.17 & 6.31 & 6.71 & 6.87 \\
\hline \multirow{12}{*}{0.1} & \multirow{4}{*}{25} & 0.05 & 3.84 & 3.95 & 4.09 & 4.04 & \multirow{12}{*}{0.2} & \multirow{4}{*}{25} & 0.05 & 4.36 & 4.27 & 4.48 & 4.37 \\
\hline & & 0.1 & 3.42 & 3.71 & 3.59 & 4.23 & & & 0.1 & 4.02 & 4.08 & 4.18 & 4.45 \\
\hline & & 0.15 & 3.36 & 3.57 & 3.79 & 4.24 & & & 0.15 & 3.82 & 3.91 & 4.09 & 4.47 \\
\hline & & 0.2 & 3.26 & 3.41 & 3.99 & 4.31 & & & 0.2 & 3.67 & 3.74 & 4.27 & 4.52 \\
\hline & \multirow{4}{*}{50} & 0.05 & 5.04 & 5.17 & 5.23 & 5.17 & & \multirow{4}{*}{50} & 0.05 & 6.31 & 6.27 & 6.44 & 6.34 \\
\hline & & 0.1 & 4.67 & 4.99 & 4.86 & 5.38 & & & 0.1 & 5.99 & 5.12 & 6.17 & 6.44 \\
\hline & & 0.15 & 4.58 & 4.78 & 4.98 & 5.36 & & & 0.15 & 5.78 & 5.89 & 6.06 & 6.52 \\
\hline & & 0.2 & 4.53 & 4.70 & 5.18 & 5.43 & & & 0.2 & 5.69 & 5.79 & 6.27 & 6.56 \\
\hline & \multirow{4}{*}{100} & 0.05 & 6.06 & 6.17 & 6.03 & 6.13 & & \multirow{4}{*}{100} & 0.05 & 7.37 & 7.29 & 7.39 & 7.31 \\
\hline & & 0.1 & 5.65 & 6.02 & 6.21 & 6.24 & & & 0.1 & 7.03 & 6.09 & 7.14 & 7.42 \\
\hline & & 0.15 & 5.61 & 5.84 & 5.56 & 6.37 & & & 0.15 & 6.82 & 6.84 & 7.09 & 7.51 \\
\hline & & 0.2 & 5.57 & 5.71 & 6.08 & 6.43 & & & 0.2 & 6.71 & 6.80 & 7.24 & 7.57 \\
\hline
\end{tabular}


Table 4.8 ARL1 Comparison for different smoothing parameters $R_{a}$ at $3 \sigma$ (SETS)

\begin{tabular}{|c|c|c|c|c|c|c|c|c|c|c|c|c|c|}
\hline \multirow[b]{2}{*}{$\mathrm{Rm}$} & \multirow[b]{2}{*}{ ARL1 } & \multirow[b]{2}{*}{$\operatorname{Re}$} & \multicolumn{4}{|c|}{ Ra } & \multirow[b]{2}{*}{$\mathbf{R m}$} & \multirow[b]{2}{*}{ ARL1 } & \multirow[b]{2}{*}{$\operatorname{Re}$} & \multicolumn{4}{|c|}{$\mathbf{R a}$} \\
\hline & & & 0.05 & 0.1 & 0.15 & 0.2 & & & & 0.05 & 0.1 & 0.15 & 0.2 \\
\hline \multirow{12}{*}{0.05} & \multirow{4}{*}{25} & $\overline{0.05}$ & 1.95 & 11.88 & $\overline{c 1.89}$ & $\overline{c 1.81}$ & \multirow{12}{*}{0.15} & \multirow{4}{*}{25} & 0.05 & 2.68 & 2.57 & 2.47 & $\overline{2.41}$ \\
\hline & & 0.1 & 1.72 & 1.71 & 1.70 & 1.64 & & & 0.1 & 2.30 & 2.26 & 2.17 & 2.12 \\
\hline & & 0.15 & 1.63 & 1.61 & 1.42 & 1.60 & & & 0.15 & 2.05 & 2.06 & 1.97 & 1.98 \\
\hline & & 0.2 & 1.59 & 1.55 & 1.49 & 1.51 & & & 0.2 & 1.90 & 1.91 & 1.83 & 1.79 \\
\hline & \multirow{4}{*}{50} & 0.05 & 2.39 & 2.29 & 2.31 & 2.19 & & \multirow{4}{*}{50} & 0.05 & 3.15 & 3.03 & 2.87 & 2.78 \\
\hline & & 0.1 & 2.16 & 2.05 & 2.09 & 2.03 & & & 0.1 & 2.68 & 2.73 & 2.70 & 2.65 \\
\hline & & 0.15 & 2.07 & 2.02 & 1.81 & 1.98 & & & 0.15 & 2.42 & 2.40 & 2.38 & 2.41 \\
\hline & & 0.2 & 1.93 & 1.83 & 1.86 & 1.87 & & & 0.2 & 2.33 & 2.31 & 2.26 & 2.23 \\
\hline & \multirow{4}{*}{100} & 0.05 & 2.97 & 2.89 & 2.91 & 2.80 & & \multirow{4}{*}{100} & 0.05 & 3.58 & 3.47 & 3.36 & 3.19 \\
\hline & & 0.1 & 2.68 & 2.64 & 2.77 & 2.63 & & & 0.1 & 3.11 & 3.17 & 3.08 & 2.98 \\
\hline & & 0.15 & 2.52 & 2.62 & 2.55 & 2.57 & & & 0.15 & 2.89 & 2.86 & 2.86 & 2.75 \\
\hline & & 0.2 & 2.31 & 2.43 & 2.45 & 2.48 & & & 0.2 & 2.76 & 2.78 & 2.71 & 2.63 \\
\hline \multirow{12}{*}{0.1} & \multirow{4}{*}{25} & 0.05 & 2.24 & 2.16 & 2.16 & 2.03 & \multirow{12}{*}{0.2} & \multirow{4}{*}{25} & 0.05 & 3.11 & 3.04 & 2.97 & 2.83 \\
\hline & & 0.1 & 2.00 & 2.04 & 1.93 & 1.87 & & & 0.1 & 2.83 & 2.71 & 2.63 & 2.59 \\
\hline & & 0.15 & 1.84 & 1.78 & 1.78 & 1.75 & & & 0.15 & 2.45 & 2.39 & 2.31 & 2.28 \\
\hline & & 0.2 & 1.73 & 1.72 & 1.71 & 1.66 & & & 0.2 & 2.23 & 2.21 & 2.12 & 2.03 \\
\hline & \multirow{4}{*}{50} & 0.05 & 2.64 & 2.53 & 2.57 & 2.45 & & \multirow{4}{*}{50} & 0.05 & 3.49 & 3.41 & 3.37 & 3.27 \\
\hline & & 0.1 & 2.43 & 2.42 & 2.34 & 2.31 & & & 0.1 & 3.22 & 3.18 & 3.05 & 2.97 \\
\hline & & 0.15 & 2.29 & 2.21 & 2.18 & 2.15 & & & 0.15 & 2.87 & 2.81 & 2.74 & 2.63 \\
\hline & & 0.2 & 2.18 & 2.23 & 2.13 & 2.03 & & & 0.2 & 2.53 & 2.57 & 2.46 & 2.48 \\
\hline & \multirow{4}{*}{100} & 0.05 & 3.11 & 2.93 & 2.99 & 2.89 & & \multirow{4}{*}{100} & 0.05 & 3.93 & 3.83 & 3.77 & 3.71 \\
\hline & & 0.1 & 2.89 & 2.77 & 2.76 & 2.74 & & & 0.1 & 3.63 & 3.55 & 3.47 & 3.45 \\
\hline & & 0.15 & 2.71 & 2.56 & 2.58 & 2.65 & & & 0.15 & 3.31 & 3.21 & 3.14 & 3.13 \\
\hline & & 0.2 & 2.64 & 2.58 & 2.57 & 2.46 & & & 0.2 & 2.94 & 2.97 & 2.85 & 3.00 \\
\hline
\end{tabular}

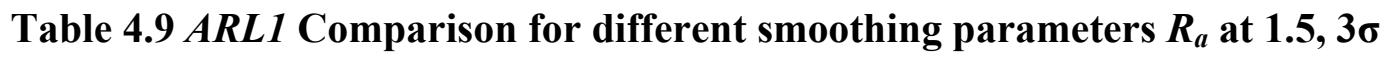
(CUSUM)

\begin{tabular}{|l|r|r|r|r|r|r|r|r|r|r|r|r|r|}
\hline Step/ & \multicolumn{1}{l|}{ Ra } & \multicolumn{3}{|c|}{$\mathbf{0 . 0 5}$} & \multicolumn{3}{|c|}{$\mathbf{0 . 1}$} & \multicolumn{3}{|c|}{$\mathbf{0 . 1 5}$} & \multicolumn{3}{|c|}{$\mathbf{0 . 2}$} \\
\hline $\mathbf{R m} /$ & \multicolumn{1}{|c|}{ ARL } & $\mathbf{2 5}$ & $\mathbf{5 0}$ & $\mathbf{1 0 0}$ & $\mathbf{2 5}$ & $\mathbf{5 0}$ & $\mathbf{1 0 0}$ & $\mathbf{2 5}$ & $\mathbf{5 0}$ & $\mathbf{1 0 0}$ & $\mathbf{2 5}$ & $\mathbf{5 0}$ & $\mathbf{1 0 0}$ \\
\hline \hline \multirow{4}{*}{$\mathbf{1 . 5}$} & $\mathbf{0 . 0 5}$ & 3.87 & 5.26 & 7.56 & 3.77 & 5.30 & 6.92 & 3.94 & 5.27 & 6.81 & 4.27 & 5.31 & 7.10 \\
& $\mathbf{0 . 1}$ & 4.27 & 6.30 & 8.76 & 4.28 & 6.15 & 7.90 & 4.29 & 5.93 & 7.62 & 4.65 & 5.92 & 7.59 \\
& $\mathbf{0 . 1 5}$ & 4.68 & 6.82 & 9.80 & 4.69 & 6.27 & 8.83 & 4.55 & 6.52 & 8.10 & 4.97 & 6.65 & 8.39 \\
& $\mathbf{0 . 2}$ & 5.17 & 7.80 & 10.72 & 4.99 & 7.09 & 9.23 & 4.97 & 6.63 & 9.05 & 5.18 & 7.19 & 8.97 \\
\hline \multirow{4}{*}{3} & $\mathbf{0 . 0 5}$ & 2.26 & 3.02 & 5.30 & 2.16 & 2.85 & 3.59 & 2.05 & 2.67 & 3.36 & 2.07 & 2.52 & 3.16 \\
\cline { 2 - 14 } & $\mathbf{0 . 1}$ & 2.63 & 4.01 & 6.05 & 2.49 & 3.65 & 4.98 & 2.37 & 3.27 & 4.35 & 2.43 & 3.16 & 3.81 \\
& $\mathbf{0 . 1 5}$ & 3.21 & 5.29 & 7.87 & 2.99 & 4.32 & 6.32 & 2.83 & 4.07 & 5.25 & 3.34 & 3.88 & 4.84 \\
& $\mathbf{0 . 2}$ & 3.89 & 6.46 & 9.40 & 3.60 & 5.53 & 7.19 & 3.34 & 4.69 & 6.24 & 3.43 & 4.44 & 5.52 \\
\hline
\end{tabular}


Table 4.10 $A R L 1$ Comparison for different smoothing parameters $R_{a}$ at $1.5 \sigma(R T S)$

\begin{tabular}{|c|c|c|c|c|c|c|c|c|c|c|c|c|c|}
\hline \multirow{2}{*}{ Rms } & \multirow{2}{*}{ ARL1 } & \multirow{2}{*}{ Rc } & \multicolumn{4}{|c|}{$\mathbf{R a}$} & \multirow{2}{*}{ Rms } & \multirow{2}{*}{ ARL1 } & \multirow{2}{*}{ Rc } & \multicolumn{4}{|c|}{$\mathbf{R a}$} \\
\hline & & & 0.05 & 0.1 & 0.15 & 0.2 & & & & 0.05 & 0.1 & 0.15 & 0.2 \\
\hline \multirow{12}{*}{0.05} & \multirow{4}{*}{25} & 0.05 & 2.03 & 1.85 & 1.62 & 1.54 & \multirow{12}{*}{0.15} & \multirow{4}{*}{25} & 0.05 & 2.24 & 1.96 & 1.91 & 1.63 \\
\hline & & 0.1 & 2.33 & 2.21 & 2.11 & 2.12 & & & 0.1 & 2.61 & 2.57 & 2.54 & 2.34 \\
\hline & & 0.15 & 2.61 & 2.39 & 2.42 & 2.44 & & & 0.15 & 3.01 & 2.96 & 2.93 & 2.71 \\
\hline & & 0.2 & 2.94 & 2.54 & 2.59 & 2.62 & & & 0.2 & 3.30 & 3.21 & 3.20 & 2.97 \\
\hline & \multirow{4}{*}{50} & 0.05 & 3.05 & 3.12 & 3.07 & 3.01 & & \multirow{4}{*}{50} & 0.05 & 3.80 & 3.54 & 3.46 & 3.37 \\
\hline & & 0.1 & 3.33 & 3.64 & 3.81 & 3.92 & & & 0.1 & 4.55 & 4.81 & 4.71 & 4.79 \\
\hline & & 0.15 & 3.70 & 3.89 & 4.26 & 4.37 & & & 0.15 & 5.34 & 5.62 & 6.17 & 5.54 \\
\hline & & 0.2 & 4.15 & 3.75 & 4.51 & 4.64 & & & 0.2 & 5.92 & 6.13 & 6.75 & 6.07 \\
\hline & \multirow{4}{*}{100} & 0.05 & 4.52 & 4.62 & 4.55 & 4.50 & & \multirow{4}{*}{100} & 0.05 & 6.83 & 6.69 & 6.58 & 6.53 \\
\hline & & 0.1 & 4.94 & 5.37 & 5.55 & 5.85 & & & 0.1 & 8.33 & 8.98 & 9.02 & 9.23 \\
\hline & & 0.15 & 5.47 & 5.74 & 6.21 & 6.52 & & & 0.15 & 9.89 & 10.89 & 11.48 & 10.83 \\
\hline & & 0.2 & 6.14 & 5.64 & 6.56 & 7.05 & & & 0.2 & 10.99 & 11.89 & 12.69 & 12.35 \\
\hline \multirow{12}{*}{0.1} & \multirow{4}{*}{25} & 0.05 & 2.20 & 1.89 & 1.84 & 1.54 & \multirow{12}{*}{0.2} & \multirow{4}{*}{25} & 0.05 & 2.29 & 2.00 & 1.96 & 1.68 \\
\hline & & 0.1 & 2.57 & 2.55 & 2.52 & 2.25 & & & 0.1 & 2.64 & 2.61 & 2.58 & 2.41 \\
\hline & & 0.15 & 2.95 & 2.91 & 2.89 & 2.65 & & & 0.15 & 3.03 & 2.99 & 2.97 & 2.78 \\
\hline & & 0.2 & 3.24 & 3.19 & 3.13 & 2.92 & & & 0.2 & 3.31 & 3.15 & 3.22 & 3.11 \\
\hline & \multirow{4}{*}{50} & 0.05 & 3.47 & 3.41 & 3.39 & 3.28 & & \multirow{4}{*}{50} & 0.05 & 3.97 & 4.04 & 4.12 & 4.08 \\
\hline & & 0.1 & 3.98 & 4.53 & 4.76 & 4.69 & & & 0.1 & 4.67 & 5.01 & 5.33 & 5.69 \\
\hline & & 0.15 & 4.51 & 5.03 & 5.51 & 5.49 & & & 0.15 & 5.67 & 5.77 & 6.15 & 6.34 \\
\hline & & 0.2 & 5.07 & 5.41 & 5.99 & 6.04 & & & 0.2 & 6.62 & 6.09 & 6.67 & 6.80 \\
\hline & \multirow{4}{*}{100} & 0.05 & 6.47 & 6.52 & 6.49 & 6.44 & & \multirow{4}{*}{100} & 0.05 & 7.13 & 7.02 & 6.99 & 6.94 \\
\hline & & 0.1 & 7.47 & 8.67 & 7.73 & 9.21 & & & 0.1 & 8.53 & 8.96 & 9.41 & 10.12 \\
\hline & & 0.15 & 8.39 & 9.67 & 9.31 & 10.80 & & & 0.15 & 10.52 & 10.46 & 10.94 & 11.62 \\
\hline & & 0.2 & 9.41 & 10.12 & 10.27 & 11.26 & & & 0.2 & 12.32 & 11.14 & 11.96 & 12.52 \\
\hline
\end{tabular}


Table 4.11 ARL1 Comparison for different smoothing parameters $R_{a}$ at $3 \sigma(R T S)$

\begin{tabular}{|c|c|c|c|c|c|c|c|c|c|c|c|c|c|}
\hline \multirow{2}{*}{ Rms } & \multirow{2}{*}{ ARL1 } & \multirow{2}{*}{ Rc } & \multicolumn{4}{|c|}{$\mathbf{R a}$} & \multirow{2}{*}{ Rms } & \multirow{2}{*}{ ARL1 } & \multirow[b]{2}{*}{ Rc } & \multicolumn{4}{|c|}{$\mathbf{R a}$} \\
\hline & & & 0.05 & 0.1 & 0.15 & 0.2 & & & & 0.05 & 0.1 & 0.15 & 0.2 \\
\hline \multirow{12}{*}{0.05} & \multirow{4}{*}{25} & 0.05 & 1.29 & 1.25 & 1.21 & 1.16 & \multirow{12}{*}{0.15} & \multirow{4}{*}{25} & 0.05 & 1.36 & 1.27 & 1.23 & 1.18 \\
\hline & & 0.1 & 1.40 & 1.31 & 1.29 & 1.28 & & & 0.1 & 1.46 & 1.40 & 1.37 & 1.33 \\
\hline & & 0.15 & 1.52 & 1.43 & 1.42 & 1.38 & & & 0.15 & 1.56 & 1.50 & 1.47 & 1.41 \\
\hline & & 0.2 & 1.63 & 1.52 & 1.43 & 1.40 & & & 0.2 & 1.67 & 1.56 & 1.51 & 1.46 \\
\hline & \multirow{4}{*}{50} & 0.05 & 1.56 & 1.51 & 1.56 & 1.17 & & \multirow{4}{*}{50} & 0.05 & 1.74 & 1.59 & 1.73 & 1.43 \\
\hline & & 0.1 & 1.71 & 1.61 & 1.67 & 1.33 & & & 0.1 & 1.87 & 1.79 & 1.82 & 1.65 \\
\hline & & 0.15 & 1.82 & 1.76 & 1.85 & 1.47 & & & 0.15 & 2.01 & 1.94 & 1.85 & 1.77 \\
\hline & & 0.2 & 1.97 & 1.87 & 1.87 & 1.51 & & & 0.2 & 2.23 & 2.06 & 1.98 & 1.84 \\
\hline & \multirow{4}{*}{100} & 0.05 & 1.80 & 1.72 & 1.81 & 1.44 & & \multirow{4}{*}{100} & 0.05 & 2.16 & 2.07 & 2.18 & 1.99 \\
\hline & & 0.1 & 2.01 & 1.86 & 1.96 & 1.66 & & & 0.1 & 2.42 & 2.47 & 2.36 & 2.43 \\
\hline & & 0.15 & 2.16 & 2.07 & 2.21 & 1.69 & & & 0.15 & 2.70 & 2.77 & 2.44 & 2.67 \\
\hline & & 0.2 & 2.37 & 2.23 & 2.24 & 1.76 & & & 0.2 & 2.96 & 3.01 & 2.71 & 2.83 \\
\hline \multirow{12}{*}{0.1} & \multirow{4}{*}{25} & 0.05 & 1.35 & 1.26 & $\overline{c 1.21}$ & 1.16 & \multirow{12}{*}{0.2} & \multirow{4}{*}{25} & 0.05 & 1.38 & 1.30 & 1.27 & 1.24 \\
\hline & & 0.1 & 1.43 & 1.38 & 1.35 & 1.32 & & & 0.1 & 1.49 & 1.45 & 1.42 & 1.34 \\
\hline & & 0.15 & 1.52 & 1.47 & 1.43 & 1.39 & & & 0.15 & 1.58 & 1.53 & 1.49 & 1.45 \\
\hline & & 0.2 & 1.62 & 1.53 & 1.47 & 1.43 & & & 0.2 & 1.71 & 1.57 & 1.55 & 1.51 \\
\hline & \multirow{4}{*}{50} & 0.05 & 1.65 & 1.56 & 1.61 & 1.34 & & \multirow{4}{*}{50} & 0.05 & 1.78 & 1.70 & 1.67 & 1.62 \\
\hline & & 0.1 & 1.77 & 1.72 & 1.84 & 1.55 & & & 0.1 & 1.92 & 1.87 & 1.79 & 1.77 \\
\hline & & 0.15 & 1.91 & 1.85 & 1.95 & 1.65 & & & 0.15 & 2.04 & 1.99 & 1.90 & 1.97 \\
\hline & & 0.2 & 2.05 & 1.94 & 2.01 & 1.71 & & & 0.2 & 2.25 & 2.05 & 1.99 & 2.13 \\
\hline & \multirow{4}{*}{100} & 0.05 & 1.95 & 1.85 & 1.97 & 1.71 & & \multirow{4}{*}{100} & 0.05 & 2.42 & 2.36 & 2.45 & 2.29 \\
\hline & & 0.1 & 2.13 & 2.09 & 2.32 & 1.89 & & & 0.1 & 2.73 & 2.66 & 2.69 & 2.59 \\
\hline & & 0.15 & 2.34 & 2.29 & 2.48 & 2.04 & & & 0.15 & 2.89 & 2.82 & 2.91 & 2.97 \\
\hline & & 0.2 & 2.55 & 2.43 & 2.57 & 2.13 & & & 0.2 & 3.31 & 3.03 & 3.09 & 3.29 \\
\hline
\end{tabular}

Tables 4.12 through 4.15 illustrate the search process used to find control limits in this research. The $w$ parameter was varied by increments of 0.1 , starting at 0.1 , since Golder and Settle's control limits indicate that the best values are usually fractional. At each value of $\mathrm{w}$, the $\mathrm{h}$ parameter was varied to find the limits yielding an $\mathrm{ARL}=25,50$ and 100 periods on unbiased errors. Using those $\mathrm{w}$ and $\mathrm{h}$ values, the ARLs to detect $1.5 \sigma$ and $3.0 \sigma$ step changes in the mean were measured. This procedure was continued until 
the ARL at $1.5 \sigma$ passed through a minimum. If there was a tie for the best limits at $1.5 \sigma$, the tie was broken with the minimum ARL at 3.0б. Gardner (1983) in his research used the same method to determine control limits. His results were replicated in this research, however, no significant differences in ARLs were found. Bias equal to $1.5 \sigma$ is about the smallest level that any tracking signal can detect in a reasonable number of periods. Since the ARL at 3.0 $\sigma$ was always two periods or less for virtually any set of limits, it seemed best to minimize the ARL at $1.5 \sigma$ rather than at $3.0 \sigma$.

Table 4.12 Backward CUSUM response comparisons, for $\boldsymbol{R}_{a}=0.05$

\begin{tabular}{|c|c|c|c|c|c|c|c|c|}
\hline \multicolumn{3}{|c|}{ unbiased errors arl = 25} & \multicolumn{3}{|c|}{ unbiased errors arl = 50} & \multicolumn{3}{|c|}{ unbiased errors arl = 100} \\
\hline \multirow{2}{*}{$\begin{array}{c}\text { Control } \\
\text { limits }(w, h)\end{array}$} & \multicolumn{2}{|c|}{ ARL to detect bias } & \multirow{2}{*}{$\begin{array}{c}\text { Control } \\
\text { limits }(w, h)\end{array}$} & \multicolumn{2}{|c|}{ ARL to detect bias } & \multirow{2}{*}{$\begin{array}{c}\text { Control } \\
\text { limits }(w, h)\end{array}$} & \multicolumn{2}{|c|}{$\overline{A R L}$ to detect bias } \\
\hline & $1.5 \sigma$ & $3.0 \sigma$ & & $1.5 \sigma$ & $3.0 \sigma$ & & $1.5 \sigma$ & $3.0 \sigma$ \\
\hline $0.1,41.2$ & 3.16 & 1.62 & $0.1,53.5$ & 4.10 & 1.99 & $0.1,63.6$ & 4.87 & 2.29 \\
\hline $0.2,16.9$ & 3.03 & 1.53 & $0.2,21.6$ & 3.83 & 1.79 & $0.2,25.4$ & 4.53 & 2.10 \\
\hline $0.3,9.5$ & 3.01 & 1.40 & $0.3,12.1$ & 3.60 & 1.68 & $0.3,14.2$ & 4.39 & 1.94 \\
\hline $0.4,6.2$ & 3.12 & 1.36 & $0.4,7.8$ & 3.80 & 1.57 & $0.4,9.3$ & 4.62 & 1.85 \\
\hline
\end{tabular}

Table 4.13 Backward CUSUM response comparisons, for $R_{a}=0.1$

\begin{tabular}{|c|c|c|c|c|c|c|c|c|}
\hline \multicolumn{3}{|c|}{ unbiased errors arl $=25$} & \multicolumn{3}{|c|}{ unbiased errors arl $=50$} & \multicolumn{3}{|c|}{ unbiased errors arl $=100$} \\
\hline \multirow{2}{*}{$\begin{array}{c}\text { Control } \\
\text { limits }(w, h)\end{array}$} & \multicolumn{2}{|c|}{ ARL to detect bias } & \multirow{2}{*}{$\begin{array}{c}\text { Control } \\
\text { limits }(w, h)\end{array}$} & \multicolumn{2}{|c|}{ ARL to detect bias } & \multirow{2}{*}{$\begin{array}{c}\text { Control } \\
\text { limits }(w, h)\end{array}$} & \multicolumn{2}{|c|}{ ARL to detect bias } \\
\hline & $1.5 \sigma$ & $3.0 \sigma$ & & $1.5 \sigma$ & $3.0 \sigma$ & & $1.5 \sigma$ & $3.0 \sigma$ \\
\hline $0.1,43.2$ & 3.12 & 1.69 & $0.1,58$ & 4.03 & 2.10 & $0.1,71.6$ & 4.89 & 2.47 \\
\hline $0.2,17.7$ & 2.97 & 1.53 & $0.2,23.1$ & 3.88 & 1.88 & $0.2,27.7$ & 4.56 & 2.15 \\
\hline $0.3,10$ & 2.89 & 1.43 & $0.3,12.6$ & 3.52 & 1.72 & $0.3,15.3$ & 4.17 & 2.03 \\
\hline $0.4,6.4$ & 2.91 & 1.37 & $0.4,8.1$ & 3.54 & 1.63 & $0.4,9.7$ & 4.27 & 1.86 \\
\hline
\end{tabular}

Table 4.14 Backward $C U S U M$ response comparisons, for $R_{a}=0.15$

\begin{tabular}{|c|c|c|c|c|c|c|c|c|}
\hline \multicolumn{3}{|c|}{ unbiased errors arl = 25} & \multicolumn{3}{|c|}{ unbiased errors arl = 50} & \multicolumn{3}{|c|}{ unbiased errors arl = 100} \\
\hline \multirow{2}{*}{$\begin{array}{c}\text { Control } \\
\text { limits }(w, h)\end{array}$} & \multicolumn{2}{|c|}{ ARL to detect bias } & \multirow{2}{*}{$\begin{array}{c}\text { Control } \\
\text { limits }(w, h)\end{array}$} & \multicolumn{2}{|c|}{ ARL to detect bias } & \multirow{2}{*}{$\begin{array}{c}\text { Control } \\
\text { limits }(w, h)\end{array}$} & \multicolumn{2}{|c|}{$\overline{A R L}$ to detect bias } \\
\hline & $1.5 \sigma$ & $3.0 \sigma$ & & $1.5 \sigma$ & $3.0 \sigma$ & & $1.5 \sigma$ & $3.0 \sigma$ \\
\hline $0.1,40.4$ & 3.31 & 1.61 & $0.1,49.7$ & 4.34 & 1.84 & $0.1,58.7$ & 4.91 & 2.17 \\
\hline $0.2,16.9$ & 3.23 & 1.53 & $0.2,20.4$ & 4.13 & 1.76 & $0.2,23.8$ & 4.77 & 1.99 \\
\hline $0.3,9.6$ & 3.34 & 1.40 & $0.3,11.6$ & 4.26 & 1.66 & $0.3,13.7$ & 5.01 & 1.92 \\
\hline $0.4,6.2$ & 3.44 & 1.36 & $0.4,7.6$ & 4.21 & 1.60 & $0.4,8.9$ & 5.23 & 1.79 \\
\hline
\end{tabular}


Table 4.15 Backward CUSUM response comparisons, for $R_{a}=0.2$

\begin{tabular}{|c|c|c|c|c|c|c|c|c|}
\hline \multicolumn{3}{|c|}{ unbiased errors arl $=25$} & \multicolumn{3}{|c|}{ unbiased errors arl $=50$} & \multicolumn{3}{|c|}{ unbiased errors arl $=100$} \\
\hline \multirow{2}{*}{$\begin{array}{c}\text { Control } \\
\text { limits }(w, h)\end{array}$} & \multicolumn{2}{|c|}{$\overline{A R L}$ to detect bias } & \multirow{2}{*}{$\begin{array}{c}\text { Control } \\
\text { limits }(w, h)\end{array}$} & \multicolumn{2}{|c|}{ ARL to detect bias } & \multirow{2}{*}{$\begin{array}{c}\text { Control } \\
\text { limits }(w, h)\end{array}$} & \multicolumn{2}{|c|}{$\overline{A R L}$ to detect bias } \\
\hline & $1.5 \sigma$ & $3.0 \sigma$ & & $1.5 \sigma$ & $3.0 \sigma$ & & $1.5 \sigma$ & $3.0 \sigma$ \\
\hline $0.1,38$ & 3.39 & 1.54 & $0.1,47.5$ & 4.32 & 1.81 & $0.1,53.9$ & 4.77 & 2.07 \\
\hline $0.2,16.3$ & 3.44 & 1.47 & $0.2,19.8$ & 4.55 & 1.73 & $0.2,22.7$ & 5.31 & 1.92 \\
\hline $0.3,9.5$ & 4.03 & 1.40 & $0.3,11.4$ & 4.77 & 1.63 & $0.3,13$ & 5.77 & 1.82 \\
\hline $0.4,6.2$ & 4.07 & 1.41 & $0.4,7.4$ & 5.23 & 1.53 & $0.4,8.7$ & 5.87 & 1.81 \\
\hline
\end{tabular}

The Control limits for the PCUSUM tracking signal were determined according to the procedure described in section 3.3.5 and is shown in Table 4.16.

Table 4.16 PCUSUM Control limits

\begin{tabular}{|c|c|c|c|}
\hline Ra \ARL1 & $\mathbf{2 5}$ & $\mathbf{5 0}$ & $\mathbf{1 0 0}$ \\
\hline \hline $\mathbf{0 . 0 5}$ & 2.59 & 2.82 & 3.03 \\
\hline $\mathbf{0 . 1}$ & 2.54 & 2.76 & 2.97 \\
\hline $\mathbf{0 . 1 5}$ & 2.51 & 2.76 & 2.97 \\
\hline $\mathbf{0 . 2}$ & 2.51 & 2.72 & 2.99 \\
\hline
\end{tabular}

\subsection{Performance Comparison}

All tracking signals were compared at control limits yielding ARLs of 25, 50 and 100 periods on unbiased errors from simple exponential smoothing. Comparisons were made using smoothed values of variance (MAD, MSE, or $\sigma^{2}$ ) of forecast errors. The tracking signals were compared at step responses of $1 \sigma, 2 \sigma$, and $3 \sigma$ and also for a random step increase. The tracking signals were compared under two different categories:

a) When all smoothing constants are equal.

b) When $R_{m}=R_{m s}=0.05$ and all other smoothing constants varied from 0.05 to 0.2 . 


\subsubsection{All smoothing constants are equal}

The comparisons are given in Tables 4.17 through 4.20. Table 4.17 gives the comparisons when all smoothing constants are equal to 0.05 . The autocorrelation signal is the best choice when all smoothing constants are equal to 0.05 . However, at an unbiased ARL of 100 and $1 \sigma B C U S U M$ is the best tracking signal.

Table 4.17 Performance comparison: All smoothing constants $=\mathbf{0 . 0 5}$

\begin{tabular}{|c|c|c|c|c|c|}
\hline Step size & CUSUM & SETS & RTS & BCUSUM & PCUSUM \\
\hline \hline 0 & 25 & 25 & 25 & 25 & 25 \\
\hline 1 & 5.64 & 5.15 & 2.85 & 4.86 & 8.51 \\
\hline 2 & 3.00 & 2.70 & 1.60 & 2.15 & 3.12 \\
\hline 2.5 & 3.10 & 1.43 & 1.24 & 1.77 & 2.64 \\
\hline 3 & 2.20 & 1.93 & 1.32 & 1.47 & 2.28 \\
\hline 0 & 50 & 50 & 50 & 50 & 50 \\
\hline 1 & 8.11 & 7.15 & 5.40 & 6.43 & 10.96 \\
\hline 2 & 4.23 & 3.45 & 2.17 & 2.61 & 3.65 \\
\hline 2.14 & 4.96 & 1.88 & 1.60 & 2.47 & 3.50 \\
\hline 3 & 3.00 & 2.39 & 1.58 & 1.72 & 2.43 \\
\hline 0 & 100 & 100 & 100 & 100 & 100 \\
\hline 1 & 10.70 & 8.68 & 9.54 & 8.12 & 12.20 \\
\hline 1.54 & 9.43 & 4.61 & 3.21 & 4.86 & 6.94 \\
\hline 2 & 5.71 & 4.09 & 2.83 & 3.00 & 3.86 \\
\hline 3 & 4.26 & 2.97 & 1.88 & 2.06 & 2.63 \\
\hline
\end{tabular}

Comparisons are given in Table 4.18 when all smoothing constants are 0.1 , for unbiased $A R L$ of 25,50 and 100 . The autocorrelation tracking signal is the best for unbiased ARL 25 at step size of $1 \sigma, 2 \sigma$ and $3 \sigma$, however, at $1.69 \sigma$ (random step) BCUSUM works best. Also, BCUSUM is the best choice for unbiased ARL of 50 and 100.

Table 4.19 gives the performance comparisons for all smoothing constants $=0.15$. The autocorrelation tracking signal is the best choice for unbiased ARL of 25 and Bacward CUSUM tracking signal is the best choice for unbiased ARL of 50 and 100. 
Table 4.18 Performance comparison: All smoothing constants $=0.1$

\begin{tabular}{|c|c|c|c|c|c|}
\hline Step size & CUSUM & SETS & RTS & BCUSUM & PCUSUM \\
\hline \hline 0 & 25 & 25 & 25 & 25 & 25 \\
\hline 1 & 6.26 & 5.72 & 3.44 & 5.57 & 10.42 \\
\hline 1.69 & 3.81 & 3.14 & 3.16 & 2.58 & 4.00 \\
\hline 2 & 3.39 & 2.63 & 1.75 & 2.07 & 3.17 \\
\hline 3 & 2.57 & 1.91 & 1.38 & 1.44 & 2.30 \\
\hline 0 & 50 & 50 & 50 & 50 & 50 \\
\hline 1 & 8.93 & 8.18 & 8.10 & 7.32 & 12.77 \\
\hline 2 & 4.75 & 3.33 & 2.73 & 2.57 & 3.65 \\
\hline 2.25 & 4.37 & 3.02 & 2.35 & 2.27 & 3.21 \\
\hline 3 & 3.67 & 2.42 & 1.76 & 1.70 & 2.42 \\
\hline 0 & 100 & 100 & 100 & 100 & 100 \\
\hline 1 & 11.86 & 9.88 & 16.61 & 9.30 & 16.18 \\
\hline 1.41 & 8.45 & 6.02 & 10.15 & 4.86 & 8.01 \\
\hline 2 & 6.32 & 4.07 & 4.61 & 3.02 & 4.22 \\
\hline 3 & 4.96 & 2.77 & 2.02 & 1.95 & 2.59 \\
\hline
\end{tabular}

Table 4.19 Performance comparison: All smoothing constants $=\mathbf{0 . 1 5}$

\begin{tabular}{|c|c|c|c|c|c|}
\hline Step size & CUSUM & SETS & RTS & BCUSUM & PCUSUM \\
\hline \hline 0 & 25 & 25 & 25 & 25 & 25 \\
\hline 1 & 7.11 & 7.13 & 4.54 & 6.60 & 14.86 \\
\hline 2 & 3.68 & 2.83 & 2.05 & 2.29 & 3.42 \\
\hline 2.61 & 3.04 & 2.21 & 1.58 & 1.72 & 2.51 \\
\hline 3 & 2.72 & 1.97 & 1.41 & 1.53 & 2.31 \\
\hline 0 & 50 & 50 & 50 & 50 & 50 \\
\hline 1 & 9.72 & 9.82 & 9.29 & 8.17 & 17.12 \\
\hline 2 & 5.08 & 3.57 & 3.80 & 2.72 & 4.44 \\
\hline 2.11 & 4.98 & 3.33 & 3.49 & 2.51 & 3.67 \\
\hline 3 & 4.02 & 2.38 & 1.87 & 1.74 & 2.44 \\
\hline 0 & 100 & 100 & 100 & 100 & 100 \\
\hline 1 & 12.34 & 11.87 & 17.57 & 9.60 & 19.54 \\
\hline 2 & 6.48 & 4.27 & 7.10 & 3.17 & 4.73 \\
\hline 2.67 & 5.55 & 3.15 & 4.04 & 2.24 & 2.95 \\
\hline 3 & 5.27 & 2.86 & 2.92 & 2.00 & 2.59 \\
\hline
\end{tabular}


When all smoothing constants are 0.2 , Autocorrelation tracking signal is the best choice at unbiased ARL of 25 and Backward CUSUM tracking signal is the best choice at unbiased ARL of 50 and 100. The results are shown in Table 4.20.

Table 4.20 Performance comparison: All smoothing constants $=0.2$

\begin{tabular}{|c|c|c|c|c|c|}
\hline Step size & CUSUM & SETS & RTS & BCUSUM & PCUSUM \\
\hline \hline 0 & 25 & 25 & 25 & 25 & 25 \\
\hline 1 & 7.69 & 9.29 & 4.44 & 6.42 & 16.10 \\
\hline 1.83 & 4.17 & 3.31 & 2.49 & 2.61 & 4.68 \\
\hline 2 & 3.85 & 2.91 & 2.27 & 2.28 & 3.97 \\
\hline 3 & 3.12 & 2.03 & 1.50 & 1.53 & 2.27 \\
\hline 0 & 50 & 50 & 50 & 50 & 50 \\
\hline 1 & 10.08 & 12.83 & 10.40 & 8.74 & 19.60 \\
\hline 1.5 & 6.85 & 6.56 & 6.44 & 4.36 & 9.95 \\
\hline 2 & 5.44 & 3.83 & 4.46 & 2.87 & 4.72 \\
\hline 3 & 4.37 & 2.48 & 2.21 & 1.81 & 2.42 \\
\hline 0 & 100 & 100 & 100 & 100 & 100 \\
\hline 1 & 14.26 & 15.07 & 19.07 & 10.49 & 22.15 \\
\hline 1.92 & 6.99 & 5.23 & 10.06 & 3.35 & 6.70 \\
\hline 2 & 6.72 & 5.33 & 9.84 & 3.17 & 5.51 \\
\hline 3 & 5.59 & 3.00 & 4.42 & 2.06 & 2.73 \\
\hline
\end{tabular}

\subsubsection{When $\boldsymbol{R}_{m}=R_{m s}=0.05$}

Based on the tests for the choice of the best smoothing parameters $R_{m}$ and $R_{m s}$, all the tracking signals were compared when $R_{m}=R_{m s}=0.05, R_{a}, R_{e}$ and $R_{c}$ were varied from 0.05 to 0.2 in steps of 0.05 . The results are shown in Tables 4.21 to 4.24. 
Table 4.21 Performance comparison: $R_{m}=R_{m s}=0.05, R_{a}=0.05$

\begin{tabular}{|c|c|c|c|c|c|c|c|c|c|c|c|}
\hline \multirow{2}{*}{ Step size } & \multirow{2}{*}{ CUSUM } & \multicolumn{9}{|c|}{ SETS } & \multicolumn{5}{|c|}{ RTS } & \multirow{2}{*}{ BCUSUM } & PCUSUM \\
\cline { 3 - 11 } & & 0.05 & 0.1 & 0.15 & 0.2 & 0.05 & 0.1 & 0.15 & 0.2 & & \\
\hline \hline 0 & 25 & 25 & 25 & 25 & 25 & 25 & 25 & 25 & 25 & 25 & 25 \\
\hline 1 & 5.64 & 5.15 & 4.82 & 4.89 & 5.00 & 2.85 & 3.50 & 3.76 & 3.75 & 4.86 & 8.51 \\
\hline 1.38 & 4.16 & 3.40 & 3.48 & 3.58 & 3.45 & 2.38 & 2.47 & 2.58 & 2.71 & 3.20 & 5.01 \\
\hline 2 & 3.00 & 2.70 & 2.45 & 2.37 & 2.30 & 1.60 & 1.76 & 1.84 & 1.87 & 2.15 & 3.12 \\
\hline 3 & 2.20 & 1.93 & 1.75 & 1.67 & 1.56 & 1.32 & 1.40 & 1.41 & 1.43 & 1.47 & 2.28 \\
\hline 0 & 50 & 50 & 50 & 50 & 50 & 50 & 50 & 50 & 50 & 50 & 50 \\
\hline 1 & 8.11 & 7.15 & 6.53 & 6.48 & 7.00 & 5.40 & 6.20 & 6.82 & 6.51 & 6.43 & 10.96 \\
\hline 2 & 4.23 & 3.45 & 3.03 & 2.93 & 2.75 & 2.17 & 2.27 & 2.39 & 2.37 & 2.61 & 3.65 \\
\hline 2.14 & 4.96 & 1.88 & 2.89 & 2.65 & 2.55 & 1.60 & 2.16 & 2.08 & 2.17 & 2.47 & 3.50 \\
\hline 3 & 3.00 & 2.39 & 2.14 & 1.96 & 1.85 & 1.58 & 1.61 & 1.61 & 1.65 & 1.72 & 2.43 \\
\hline 0 & 100 & 100 & 100 & 100 & 100 & 100 & 100 & 100 & 100 & 100 & 100 \\
\hline 1 & 10.70 & 8.68 & 9.07 & 8.99 & 8.76 & 9.54 & 10.28 & 10.54 & 10.72 & 8.12 & 12.20 \\
\hline 1.54 & 9.43 & 4.61 & 4.76 & 4.48 & 4.40 & 3.21 & 4.66 & 4.75 & 4.79 & 4.86 & 6.94 \\
\hline 2 & 5.71 & 4.09 & 3.61 & 3.37 & 3.13 & 2.83 & 2.81 & 2.88 & 2.74 & 3.00 & 3.86 \\
\hline 3 & 4.26 & 2.97 & 2.46 & 3.34 & 2.06 & 1.88 & 1.83 & 2.82 & 1.79 & 2.06 & 2.63 \\
\hline
\end{tabular}

Table 4.21 presents the performance comparison of all the tracking signals when $R_{m}=R_{m s}=0.05, R_{a}=0.05, R_{e}$ and $R_{c}$ are varied from 0.05 to 0.2 in steps of 0.05 . The autocorrelation signal with $R_{c}=0.05$ is the best when unbiased ARL is 25 and 50 . At unbiased ARL of 100 and a step of $1 \sigma$ Backward CUSUM is the best, and at steps of $1.54 \sigma, 2 \sigma$ and $3 \sigma$ autocorrelation tracking signal with $R_{c}=0.05$ is the best.

Table 4.22 presents the performance comparison when $R_{m}=R_{m s}=0.05, R_{a}=0.1, R_{e}$ and $R_{c}$ varied from 0.05 to 0.02 . At unbiased ARL's of 25 and 50 the autocorrelation signal with $R_{c}=0.05$ is the best. At unbiased ARL of 100 , Backward CUSUM tracking signal is the best at step sizes of $1 \sigma, 1.39 \sigma$ and $2 \sigma$. However, at $3 \sigma$ autocorrelation signal is the best. 
Table 4.22 Performance comparison: $R_{m}=R_{m s}=0.05, R_{a}=0.1$

\begin{tabular}{|c|c|c|c|c|c|c|c|c|c|c|c|}
\hline \multirow{2}{*}{ Step size } & \multirow{2}{*}{ CUSUM } & \multicolumn{4}{|c|}{ SETS } & \multicolumn{4}{|c|}{ RTS } & \multirow{2}{*}{ BCUSUM } & \multirow{2}{*}{ PCUSUM } \\
\hline & & 0.05 & 0.1 & 0.15 & 0.2 & 0.05 & 0.1 & 0.15 & 0.2 & & \\
\hline 0 & 25 & 25 & $\bar{~} 25$ & 25 & $\bar{~} 25$ & 25 & $\bar{~} 25$ & 25 & $\bar{~} 25$ & 25 & 25 \\
\hline 1 & 5.92 & 5.57 & 5.64 & 5.39 & 5.83 & 2.45 & 3.10 & 4.04 & 4.02 & 5.72 & 11.60 \\
\hline 2 & 2.99 & 2.68 & 2.56 & 2.35 & 2.27 & 1.56 & 1.72 & 1.76 & 1.83 & 2.15 & 3.30 \\
\hline 2.22 & 2.71 & 2.49 & 2.31 & 2.11 & 2.06 & 1.45 & 1.56 & 1.70 & 1.72 & 1.89 & 2.87 \\
\hline 3 & 2.06 & 1.86 & 1.76 & 1.61 & 1.55 & 1.28 & 1.34 & 1.39 & 1.42 & 1.40 & 2.27 \\
\hline 0 & 50 & 50 & 50 & 50 & 50 & 50 & 50 & 50 & 50 & 50 & 50 \\
\hline 1 & 8.41 & 7.70 & 7.79 & 8.04 & 8.76 & 4.73 & 6.02 & 7.30 & 8.06 & 7.84 & 13.54 \\
\hline 1.77 & 4.48 & 3.76 & 3.51 & 3.37 & 3.06 & 2.38 & 2.62 & 2.95 & 3.28 & 3.03 & 4.64 \\
\hline 2 & 4.11 & 3.30 & 3.00 & 2.86 & 2.69 & 2.06 & 2.19 & 2.35 & 2.51 & 2.55 & 3.59 \\
\hline 3 & 2.85 & 2.30 & 2.05 & 1.90 & 1.76 & 1.52 & 1.55 & 1.60 & 1.59 & 1.71 & 2.43 \\
\hline 0 & 100 & 100 & 100 & 100 & 100 & 100 & 100 & 100 & 100 & 100 & 100 \\
\hline 1 & 10.68 & 9.72 & 10.03 & 9.77 & 10.91 & 11.06 & 12.94 & 13.92 & 14.47 & 9.52 & 16.16 \\
\hline 1.39 & 7.71 & 6.15 & 5.83 & 5.91 & 6.77 & 7.59 & 7.49 & 8.74 & 8.47 & 5.63 & 8.89 \\
\hline 2 & 5.09 & 3.88 & 3.61 & 3.41 & 3.25 & 3.10 & 3.60 & 3.30 & 3.36 & 3.07 & 4.23 \\
\hline 3 & 3.62 & 2.69 & 2.34 & 2.18 & 2.03 & 1.83 & 1.86 & 1.87 & 1.79 & 1.94 & 2.58 \\
\hline
\end{tabular}

The performance comparison when $R_{m}=R_{m s}=0.05, R_{a}=0.15, R_{e}$ and $R_{c}$ are varied from 0.05 to 0.2 in steps of 0.05 is presented in Table 4.23. The autocorrelation tracking signal with $R_{c}=0.05$ is the best choice for all the scenarios.

Table 4.23 Performance comparison: $\boldsymbol{R}_{m}=\boldsymbol{R}_{m s}=0.05, \boldsymbol{R}_{a}=\mathbf{0 . 1 5}$

\begin{tabular}{|c|c|c|c|c|c|c|c|c|c|c|c|}
\hline \multirow{2}{*}{ Step size } & \multirow{2}{*}{ CUSUM } & \multicolumn{9}{|c|}{ SETS } & \multicolumn{5}{|c|}{ RTS } & \multirow{2}{*}{ BCUSUM } & PCUSUM \\
\cline { 3 - 11 } & & 0.05 & 0.1 & 0.15 & 0.2 & 0.05 & 0.1 & 0.15 & 0.2 & & \\
\hline \hline 0 & 25 & 25 & 25 & 25 & 25 & 25 & 25 & 25 & 25 & 25 & 25 \\
\hline 1 & 6.27 & 5.92 & 6.14 & 6.90 & 7.11 & 2.10 & 2.80 & 3.55 & 3.94 & 5.98 & 13.58 \\
\hline 2 & 2.89 & 2.68 & 2.50 & 2.45 & 2.33 & 1.41 & 1.65 & 1.78 & 1.83 & 2.26 & 3.43 \\
\hline 2.31 & 2.58 & 2.33 & 2.19 & 2.03 & 1.94 & 1.33 & 1.46 & 1.52 & 1.69 & 2.00 & 2.85 \\
\hline 3 & 2.02 & 1.80 & 1.70 & 1.62 & 1.56 & 1.18 & 1.28 & 1.33 & 1.42 & 1.49 & 2.28 \\
\hline 0 & 50 & 50 & 50 & 50 & 50 & 50 & 50 & 50 & 50 & 50 & 50 \\
\hline 1 & 8.89 & 8.45 & 8.61 & 9.34 & 11.04 & 4.29 & 6.54 & 8.14 & 7.43 & 8.58 & 17.39 \\
\hline 2 & 3.98 & 3.34 & 2.88 & 2.97 & 2.87 & 1.97 & 2.33 & 3.28 & 2.54 & 2.66 & 4.08 \\
\hline 2.13 & 3.68 & 3.07 & 2.18 & 2.70 & 2.59 & 1.86 & 2.12 & 2.44 & 2.53 & 2.53 & 3.65 \\
\hline 3 & 2.74 & 2.23 & 1.96 & 1.90 & 1.77 & 1.48 & 1.53 & 1.64 & 1.59 & 1.77 & 2.47 \\
\hline 0 & 100 & 100 & 100 & 100 & 100 & 100 & 100 & 100 & 100 & 100 & 100 \\
\hline 1 & 11.34 & 10.10 & 10.05 & 11.85 & 12.54 & 3.51 & 5.26 & 5.77 & 7.00 & 9.36 & 19.46 \\
\hline 1.77 & 5.59 & 4.38 & 4.05 & 4.14 & 4.23 & 1.97 & 2.58 & 2.76 & 3.03 & 3.60 & 5.85 \\
\hline 2 & 3.80 & 1.75 & 3.55 & 3.50 & 3.17 & 1.75 & 2.34 & 2.39 & 2.44 & 3.11 & 4.92 \\
\hline 3 & 3.38 & 2.51 & 2.23 & 2.05 & 2.02 & 1.35 & 1.49 & 1.51 & 1.55 & 2.01 & 2.62 \\
\hline
\end{tabular}


Table 4.24 Performance comparison: $R_{m}=R_{m s}=0.05, R_{a}=0.2$

\begin{tabular}{|c|c|c|c|c|c|c|c|c|c|c|c|}
\hline \multirow{2}{*}{ Step size } & \multirow{2}{*}{ CUSUM } & \multicolumn{9}{|c|}{ SETS } & \multicolumn{5}{|c|}{ RTS } & \multirow{2}{*}{ BCUSUM } & \multirow{2}{*}{ PCUSUM } \\
\cline { 3 - 11 } & & 0.05 & 0.1 & 0.15 & 0.2 & 0.05 & 0.1 & 0.15 & 0.2 & & \\
\hline \hline 0 & 25 & 25 & 25 & 25 & 25 & 25 & 25 & 25 & 25 & 25 & 25 \\
\hline 1 & 7.26 & 7.09 & 7.42 & 7.83 & 9.18 & 2.01 & 3.01 & 3.60 & 3.96 & 6.95 & 16.32 \\
\hline 1.17 & 5.78 & 5.55 & 5.70 & 6.01 & 6.24 & 1.79 & 2.64 & 3.15 & 3.30 & 5.33 & 12.59 \\
\hline 2 & 3.02 & 2.63 & 2.59 & 2.43 & 2.42 & 1.35 & 1.69 & 1.87 & 1.87 & 2.30 & 3.79 \\
\hline 3 & 2.09 & 1.82 & 1.66 & 1.59 & 1.56 & 1.16 & 1.28 & 1.31 & 1.38 & 1.56 & 2.29 \\
\hline 0 & 50 & 50 & 50 & 50 & 50 & 50 & 50 & 50 & 50 & 50 & 50 \\
\hline 1 & 9.00 & 9.24 & 9.60 & 11.40 & 11.30 & 3.71 & 6.09 & 6.39 & 6.94 & 8.73 & 20.50 \\
\hline 2 & 3.87 & 3.28 & 3.07 & 2.94 & 3.32 & 1.85 & 2.58 & 2.47 & 3.10 & 2.89 & 4.94 \\
\hline 2.53 & 3.01 & 2.55 & 2.36 & 2.17 & 2.09 & 1.51 & 1.83 & 1.99 & 1.94 & 2.17 & 2.93 \\
\hline 3 & 2.59 & 2.15 & 1.93 & 1.87 & 1.73 & 1.38 & 1.54 & 1.56 & 1.59 & 1.86 & 2.50 \\
\hline 0 & 100 & 100 & 100 & 100 & 100 & 100 & 100 & 100 & 100 & 100 & 100 \\
\hline 1 & 13.70 & 10.30 & 11.88 & 13.49 & 18.93 & 7.65 & 12.37 & 13.35 & 15.90 & 9.64 & 23.35 \\
\hline 1.69 & 6.06 & 4.92 & 4.54 & 4.76 & 5.41 & 3.97 & 6.33 & 7.91 & 8.86 & 4.27 & 9.57 \\
\hline 2 & 5.14 & 3.97 & 3.56 & 3.66 & 3.64 & 3.18 & 4.77 & 4.60 & 5.03 & 3.23 & 6.00 \\
\hline 3 & 3.12 & 2.35 & 2.94 & 2.06 & 1.95 & 1.62 & 1.90 & 1.98 & 1.94 & 2.00 & 2.66 \\
\hline
\end{tabular}

Table 4.24 presents the performance comparison when $R_{m}=R_{m s}=0.05, R_{a}=0.1, R_{e}$ and $R_{c}$ varied from 0.05 to 0.02 . At unbiased ARL's of 25, 50 and 100 the autocorrelation signal with $R_{c}=0.05$ is the best.

\subsection{True alarms: Response to a change in demand}

Average run length is not appropriate as a measure of response to a step in the average level of demand (McClain, 1988). The reason it is inappropriate is based on a real life situation a manager faces. If a step in demand has occurred recently, the manager will take immediate action to minimize the effects of the erroneous forecasts that will have occurred since the change. However, if the run length is too long, the opportunity to overcome past errors is lost; the relevant actions would be too far in the past and the current forecast will have already adjusted to the change in demand. Therefore, a measure of responsiveness should not distinguish among long runs before detection since they are 
of equal utility. Unfortunately, the average run length is influenced greatly by distribution of long runs.

Table 4.25 Response to a 1 sigma step $R_{m}=R_{m s}=0.05, R_{a}=0.1$, Unbiased ARL=50

\begin{tabular}{|c|c|c|c|c|c|c|c|c|c|c|}
\hline & \multicolumn{3}{|c|}{ Method } & \multicolumn{2}{|c|}{$\begin{array}{c}\text { Control } \\
\text { Limit }\end{array}$} & ARL & \multicolumn{2}{|c|}{ SDRL } & & \\
\hline & \multicolumn{3}{|c|}{ CUSUM } & \multicolumn{2}{|c|}{6.2} & 8.41 & \multicolumn{2}{|c|}{6.07} & & \\
\hline & \multicolumn{3}{|c|}{ SETS $(\operatorname{Re}=0.2)$} & \multicolumn{2}{|c|}{0.737} & 8.76 & \multicolumn{2}{|c|}{11.28} & & \\
\hline & \multicolumn{3}{|c|}{ RTS(Rc=0.2) } & \multicolumn{2}{|c|}{0.116} & 8.06 & \multicolumn{2}{|c|}{10.09} & & \\
\hline & \multicolumn{3}{|c|}{ BCUSUM } & \multicolumn{2}{|c|}{$0.3,12.1$} & 7.84 & \multicolumn{2}{|c|}{9.91} & & \\
\hline & \multicolumn{3}{|c|}{ PCUSUM } & \multicolumn{2}{|c|}{2.76} & 13.54 & \multicolumn{2}{|c|}{16.26} & & \\
\hline \multirow[b]{2}{*}{ Method } & \multicolumn{10}{|c|}{ Trips within $\mathrm{N}$ periods } \\
\hline & 1 & 2 & 3 & 4 & 5 & 6 & 7 & 8 & 9 & 10 \\
\hline CUSUM & 35 & 80 & 140 & 231 & 338 & 428 & 521 & 609 & 689 & 743 \\
\hline $\mathrm{SETS}(\mathrm{Re}=0.2)$ & 46 & 163 & 269 & 385 & 507 & 601 & 668 & 719 & 750 & 779 \\
\hline $\mathrm{RTS}(\mathrm{Rc}=0.2)$ & 173 & 329 & 462 & 554 & 624 & 677 & 711 & 748 & 779 & 794 \\
\hline BCUSUM & 53 & 176 & $\overline{316}$ & 437 & 545 & 621 & 677 & 735 & 761 & 796 \\
\hline PCUSUM & 25 & 88 & 181 & 262 & 339 & 404 & 453 & 504 & 530 & 566 \\
\hline & \multicolumn{10}{|c|}{ Trips within $\mathrm{N}$ periods } \\
\hline Method & 11 & 12 & 13 & 14 & 15 & 16 & 17 & 18 & 19 & 20 \\
\hline CUSUM & 799 & 844 & 874 & 894 & 910 & 932 & 942 & 953 & 956 & 962 \\
\hline SETS(Re=0.2) & 801 & 825 & 834 & 850 & 861 & 870 & 877 & 884 & 892 & 893 \\
\hline $\mathrm{RTS}(\mathrm{Rc}=0.2)$ & 807 & 825 & 839 & 846 & 853 & 862 & 871 & 878 & 886 & 890 \\
\hline BCUSUM & 816 & 834 & 855 & \begin{tabular}{|l|l|}
5661 \\
\end{tabular} & 869 & 878 & 883 & 888 & 892 & 894 \\
\hline PCUSUM & 594 & 609 & 623 & \begin{tabular}{|l|l|}
637 \\
\end{tabular} & 653 & 661 & 668 & 678 & 686 & 694 \\
\hline
\end{tabular}

To illustrate the significance of the choice of an appropriate measure, Table 4.25 shows the results of a set of experiments in which all the tracking signals were subjected to a step in demand at period 46. First, note that the average run length to detection is 8.41 for CUSUM that is faster than 8.76 periods for smoothed error or 13.54 for PCUSUM. However, it is not until 12 periods that CUSUM surpasses smoothed error signal and not until 6 periods that CUSUM surpasses PCUSUM. Clearly, the average is misleading. Smoothed error is best at detecting errors while they are still relevant.

An alternative criterion is to specify a time cutoff and a corresponding probability. For example, a manager might prefer to maximize the probability of a trip 
occurring within 5 periods of the step in demand. This has the advantage of allowing the user to decide how long is too late to detect an error.

\subsection{Performance of tracking signals: A test of responsiveness}

The criterion for this test is percent of trips detected within $\mathrm{N}$ periods holding constant the average run length between false alarms (ARL1). All tracking signals used $R_{m} / R_{m s}=0.05$ since we already determined that all tracking signals work best at $R_{m} / R_{m s}=0.05$. The forecasting model, the smoothed error tracking signal and the autocorrelation tracking signal used the values $0.05,0.1,0.15$ and 0.2 as smoothing constants for the numerator.

In Figure 4.1 the number of trips that occurred within 5 periods of the actual step in demand is shown for each tracking signal over a range of values of $R_{a}$, the forecast's smoothing parameter. The average run length $(A R L 1)$ is held constant at 25 . This shows very clearly that all of the auto correlation methods dominate all the other tracking signals. As the forecasting smoothing parameter $\left(R_{a}\right)$ increases the number of trips also increases for the autocorrelation tracking signal. The backward CUSUM tracking signal is second best when $R_{a}=0.05$ and 0.1 , and third best when $R_{a}=0.15$ and 0.12 . The smoothed error tracking signal methods clearly dominate the CUSUM and PCUSUM tracking signals. 


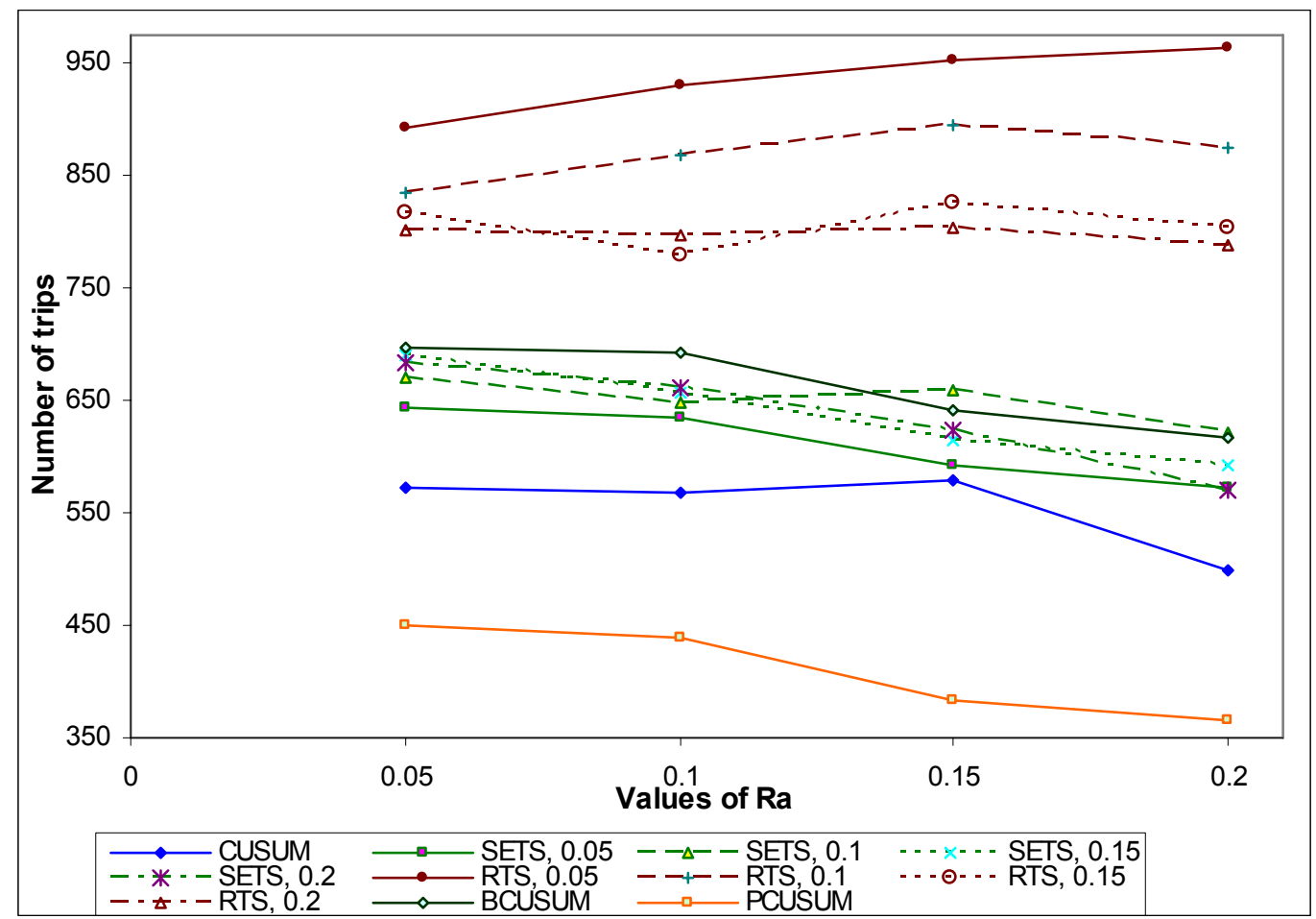

Figure 4.1 Fifth Period Response to a $1 \sigma \operatorname{step}(\mathrm{ARL}=25)$

Figure 4.2 shows what happens for a range of $\mathrm{N}$ values (allowable number of periods before detection) when $R_{a}=0.05$ and $A R L=25$ for a $1 \sigma$ step. Note that CUSUM gets of to a slow start for low $\mathrm{N}$ values but eventually catches up and overtakes smoothed error tracking signals. The autocorrelation tracking signals dominates all the other tracking signals. Figures 4.3 to 4.5 shows response within $N$ periods for $A R L=25,1 \sigma$ step and for $R_{a}$ values of $0.1,0.15$ and 0.2 respectively. It can be seen that results are similar for all the values of $R_{a}$ except for it is spread out at higher values of $R_{a}$ and $N$. 


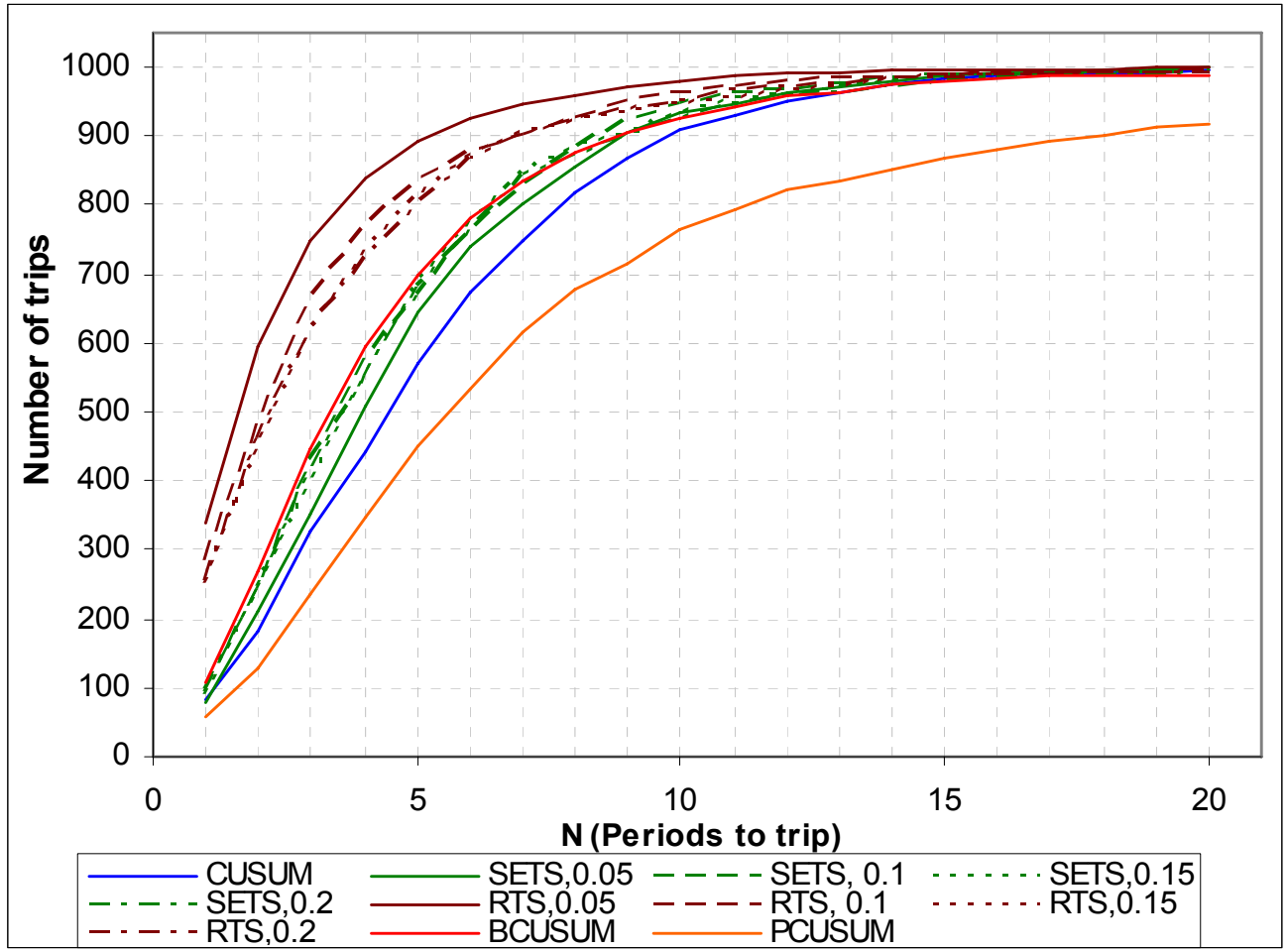

Figure 4.2 Response within $N$ periods, $A R L=25, R_{a}=0.05,1 \sigma$ step

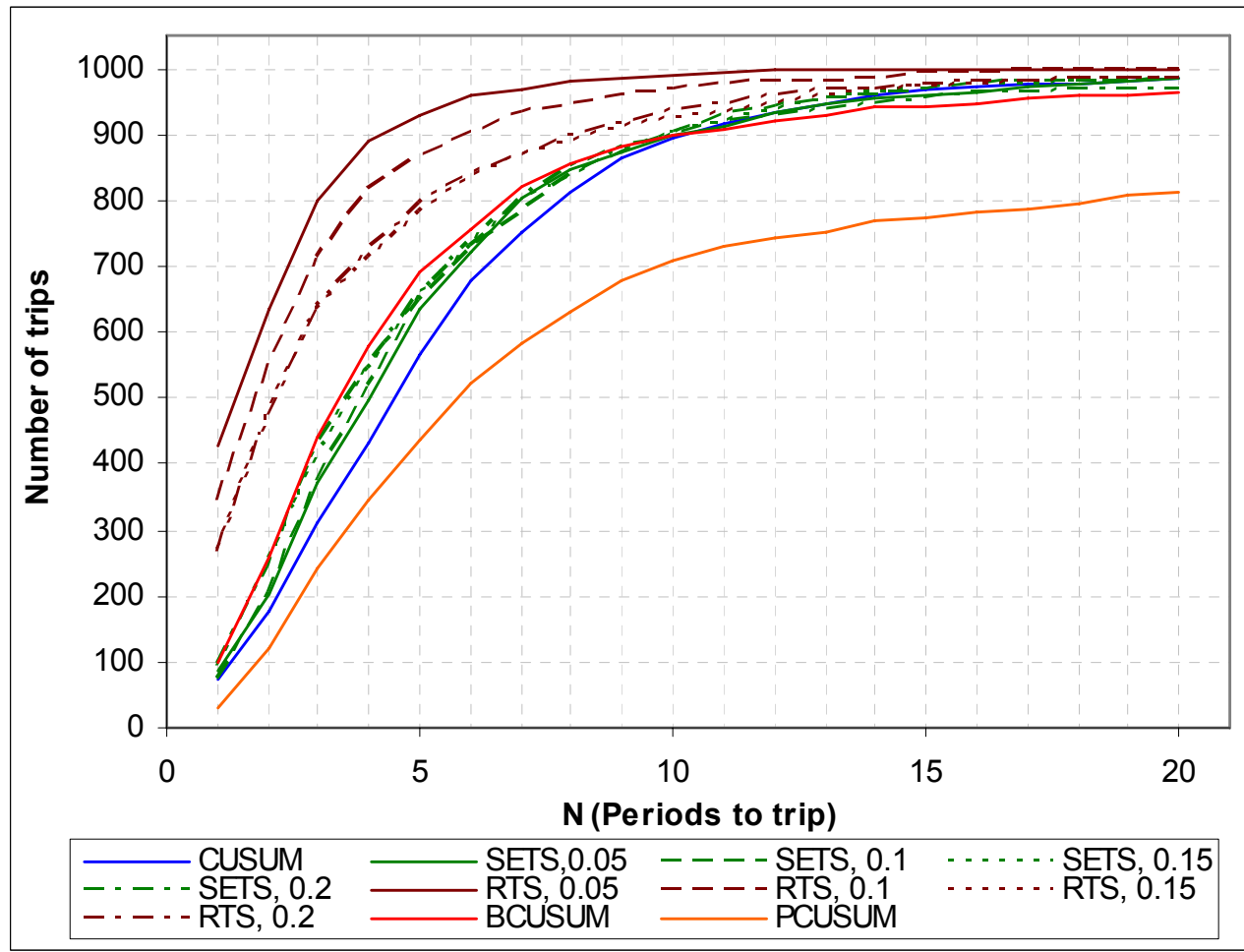

Figure 4.3 Response within $N$ periods, $A R L=25, R a=0.1,1 \sigma$ step 


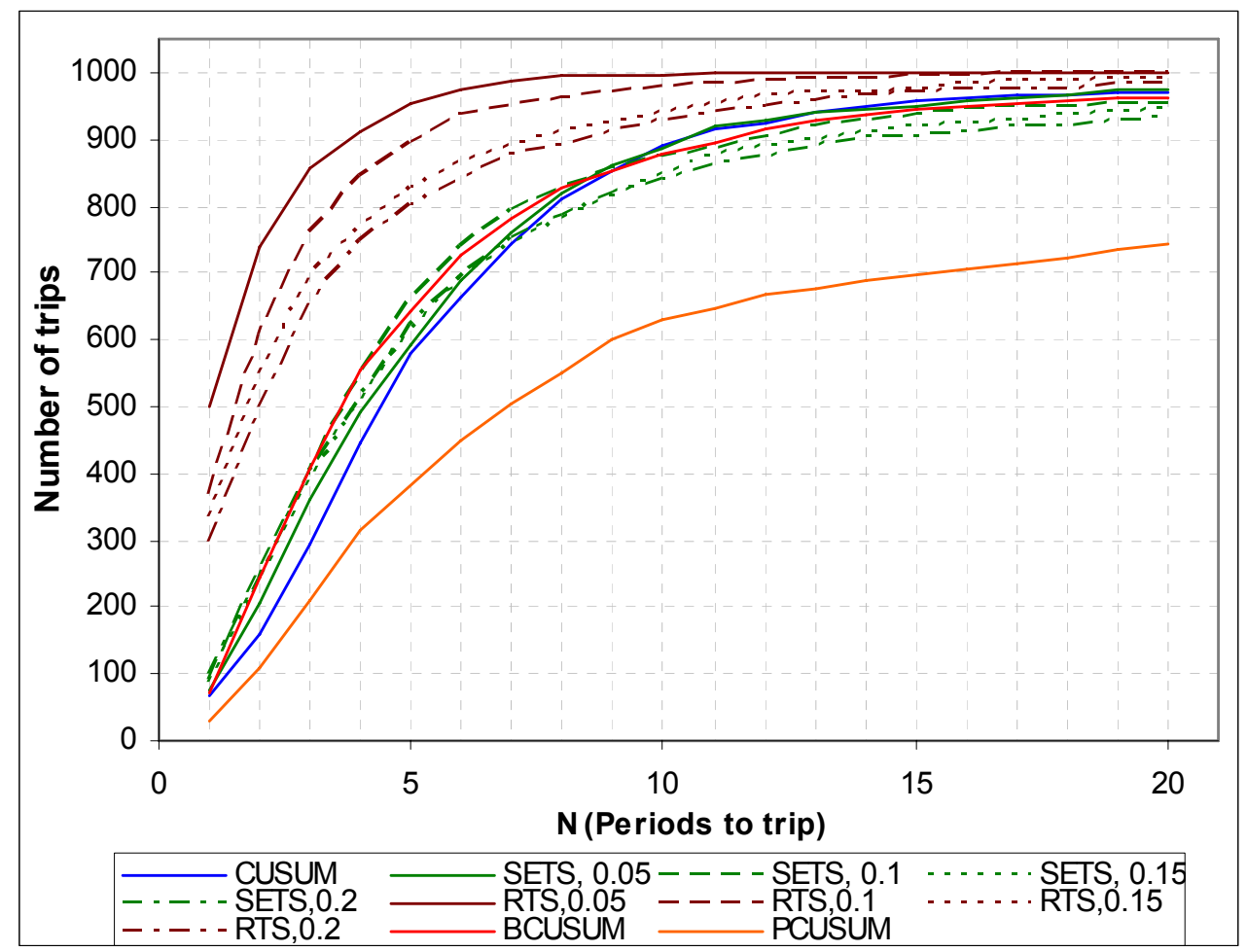

Figure 4.4 Response within $N$ periods, $A R L=25, R_{a}=0.15,1 \sigma$ step

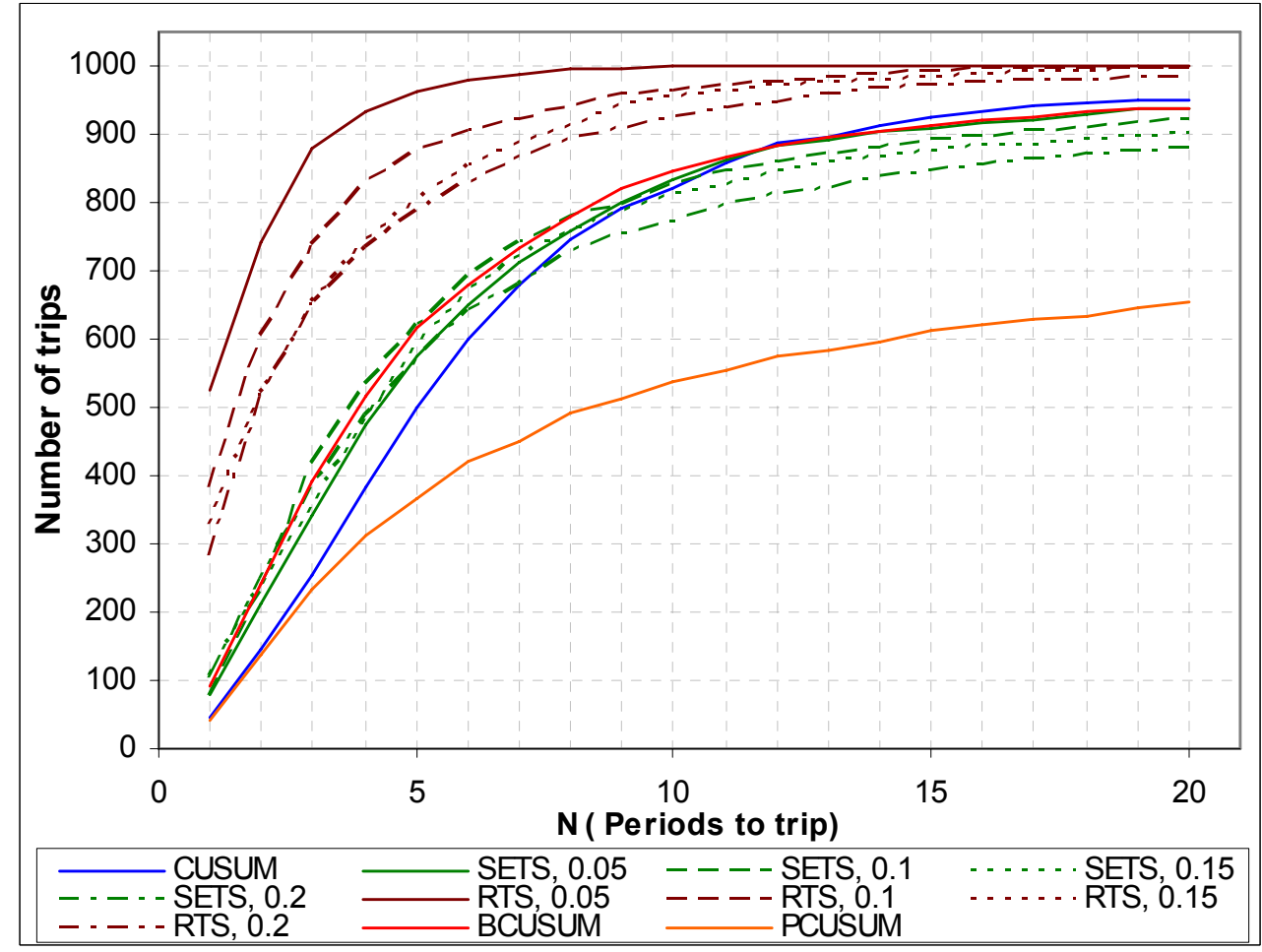

Figure 4.5 Response within $N$ periods, $A R L=25, R_{a}=0.2,1 \sigma$ step 


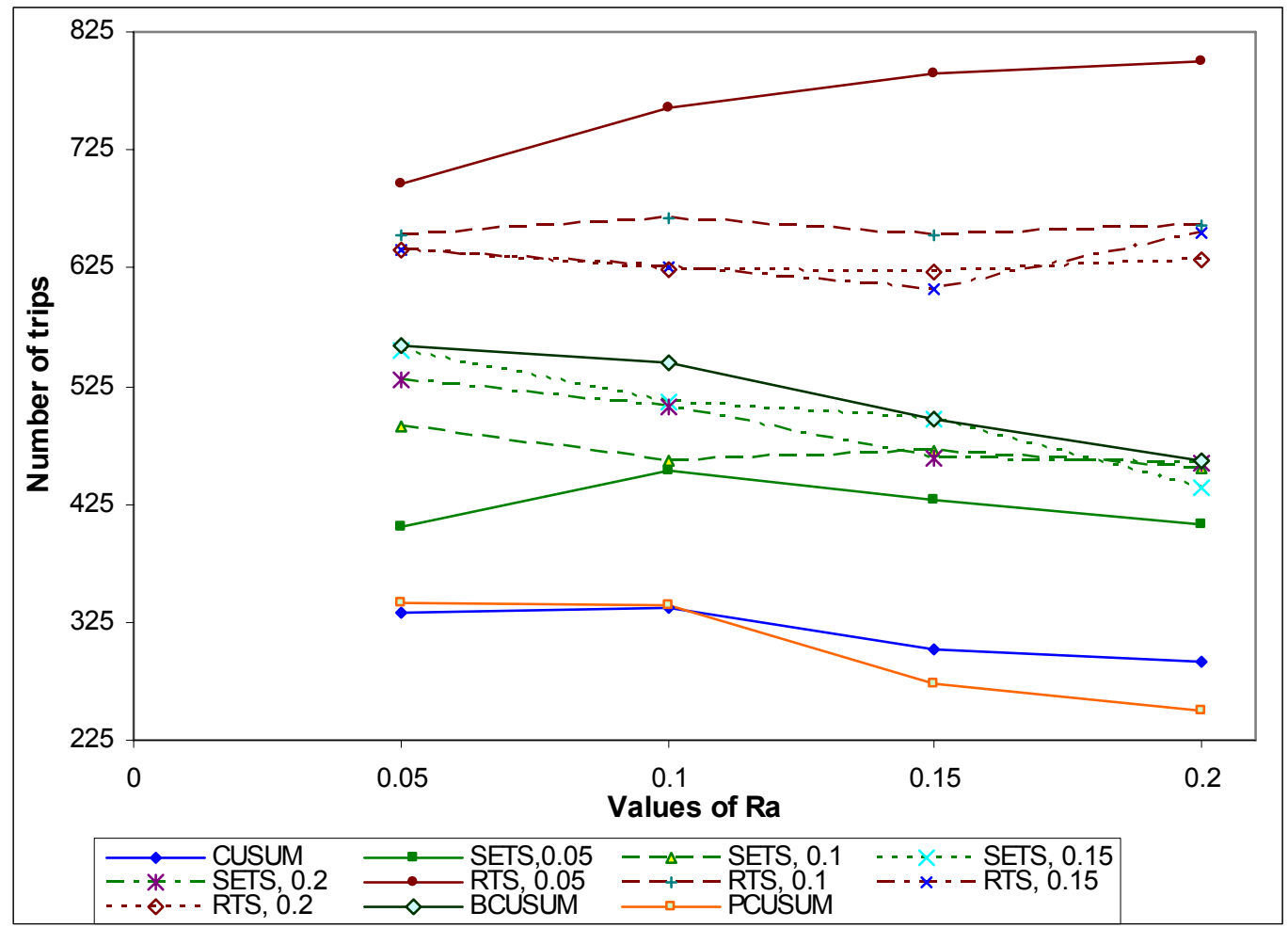

Figure 4.6 Fifth Period Response to a $1 \sigma$ step $(\mathrm{ARL}=50)$

Figure 4.6 presents the number of trips that occurred within 5 periods of the actual step in demand for each tracking signal over a range of values of $R_{a}$, the average run length $(A R L 1)$ is held constant at 50. This shows very clearly that all of the autocorrelation methods dominate all the other tracking signals. For autocorrelation tracking signal with $R_{c}=0.05$, as the forecasting smoothing parameter $\left(R_{a}\right)$ increases the number of trips also increases for the autocorrelation tracking signal. For the other values of $R_{c}$ the number of trips remains fairly constant. The backward CUSUM tracking signal is second best, the smoothed error tracking signal methods clearly dominate the CUSUM and PCUSUM tracking signals. As the forecasting smoothing parameter $\left(R_{a}\right)$ increases the number of trips decreases for all the tracking signals except for the autocorrelation tracking signal. 
Figure 4.7 shows what happens for a range of $\mathrm{N}$ values (allowable number of periods before detection) when $R_{a}=0.05$ and $A R L=50$ for a 1 sigma step. Note that CUSUM gets of to a slow start for low $\mathrm{N}$ values but eventually catches up and overtakes most of the other tracking signals. The autocorrelation tracking signals dominates all the other tracking signals for the first 8 periods. The smoothed error tracking signals starts slower then the autocorrelation tracking signals and overtakes them after $8-10$ periods.

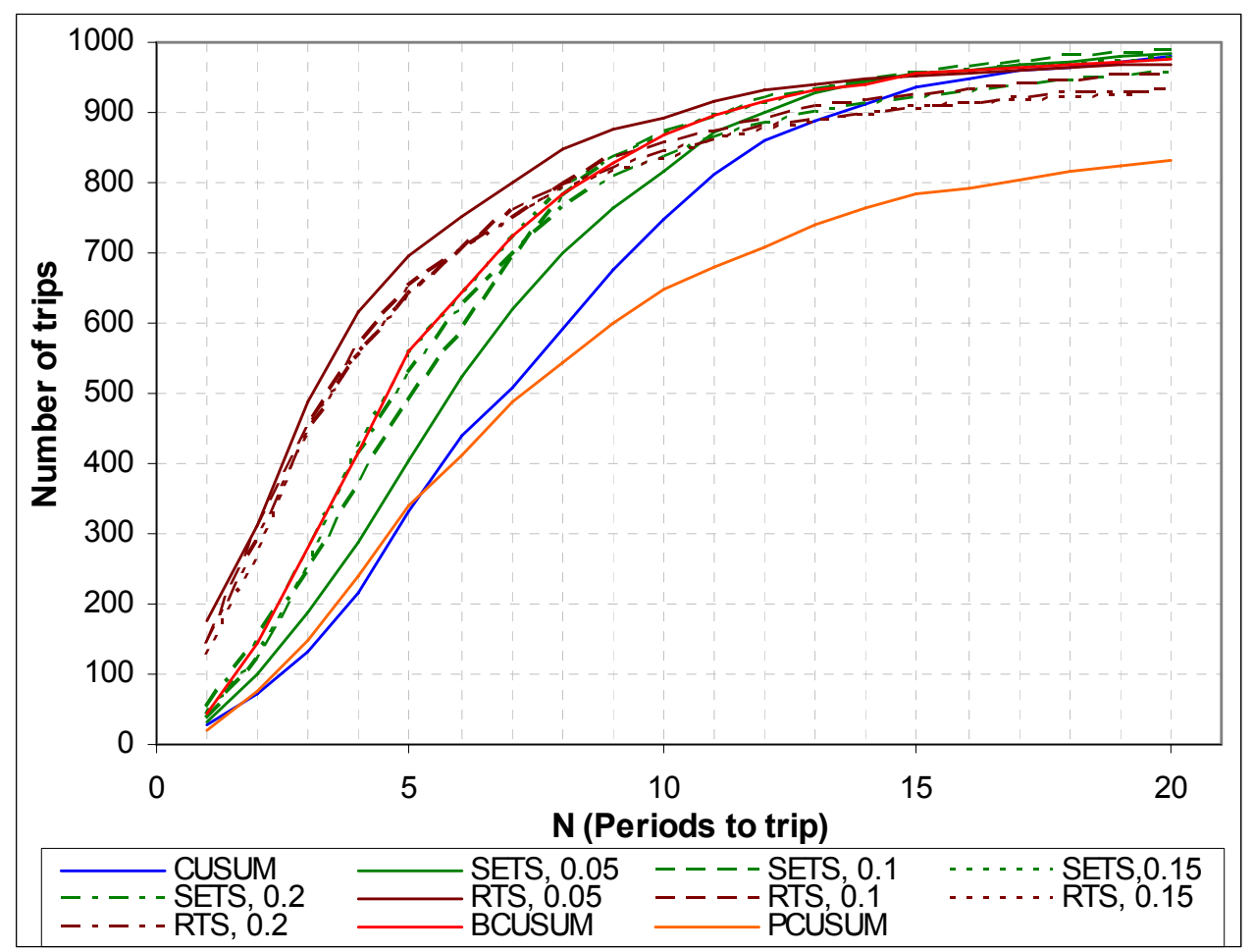

Figure 4.7 Response within $\mathrm{N}$ periods, $\mathrm{ARL}=\mathbf{5 0}, R_{a}=0.05,1 \sigma$ step

Figures 4.8 and 4.9 shows response within $N$ periods for $A R L=50,1 \sigma$ step and for $R_{a}$ values of 0.1 and 0.15 respectively. It can be seen that autocorrelation signal with $R_{c}=$ 0.05 dominates all the other tracking signals. The CUSUM tracking signal starts off slowly but eventually overtakes all the other tracking signals except for the autocorrelation tracking signal with $R_{c}=0.05$. Figure 4.10 presents the response of all tracking signals within $\mathrm{N}$ periods for $R_{a}=0.2$. It can be noted that as the forecasting 
parameter $R_{a}$ and $N$ increases, the performance of autocorrelation tracking signals improves where as the performance of smoothed error tracking signals deteriorates.

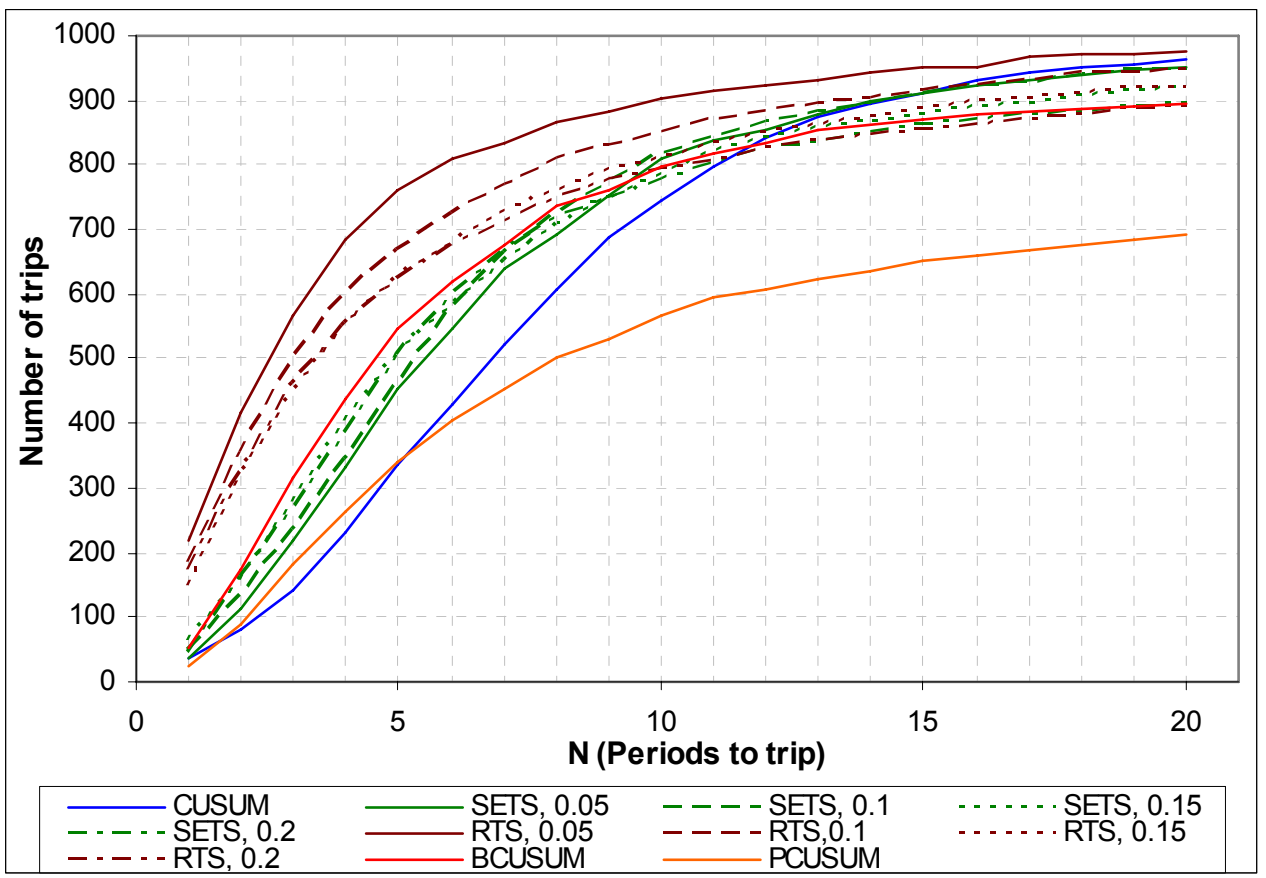

Figure 4.8 Response within $\mathrm{N}$ periods, $\mathrm{ARL}=50, R_{a}=0.1,1 \sigma$ step

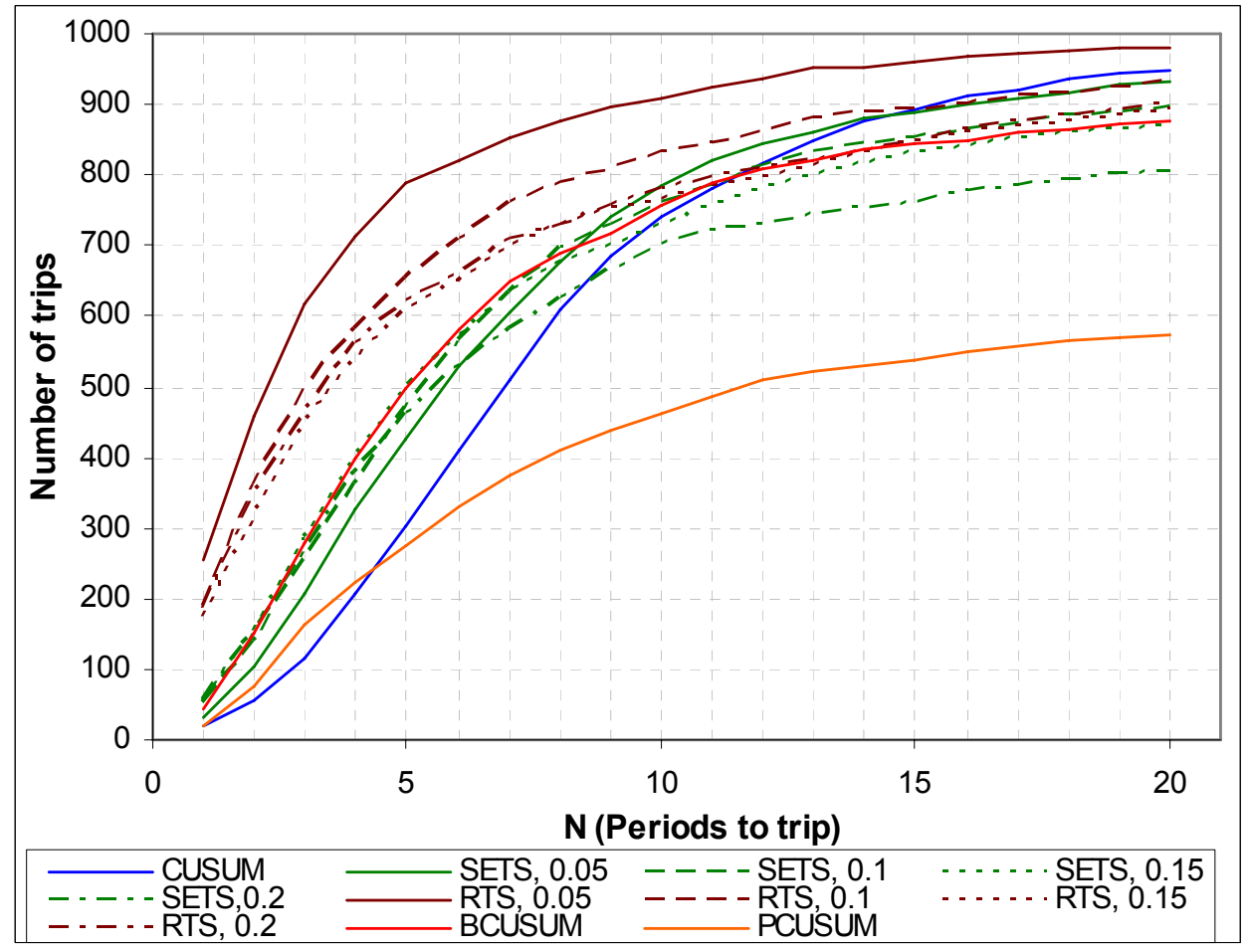

Figure 4.9 Response within $\mathrm{N}$ periods, $\mathrm{ARL}=50, R_{a}=0.15,1 \sigma$ step 


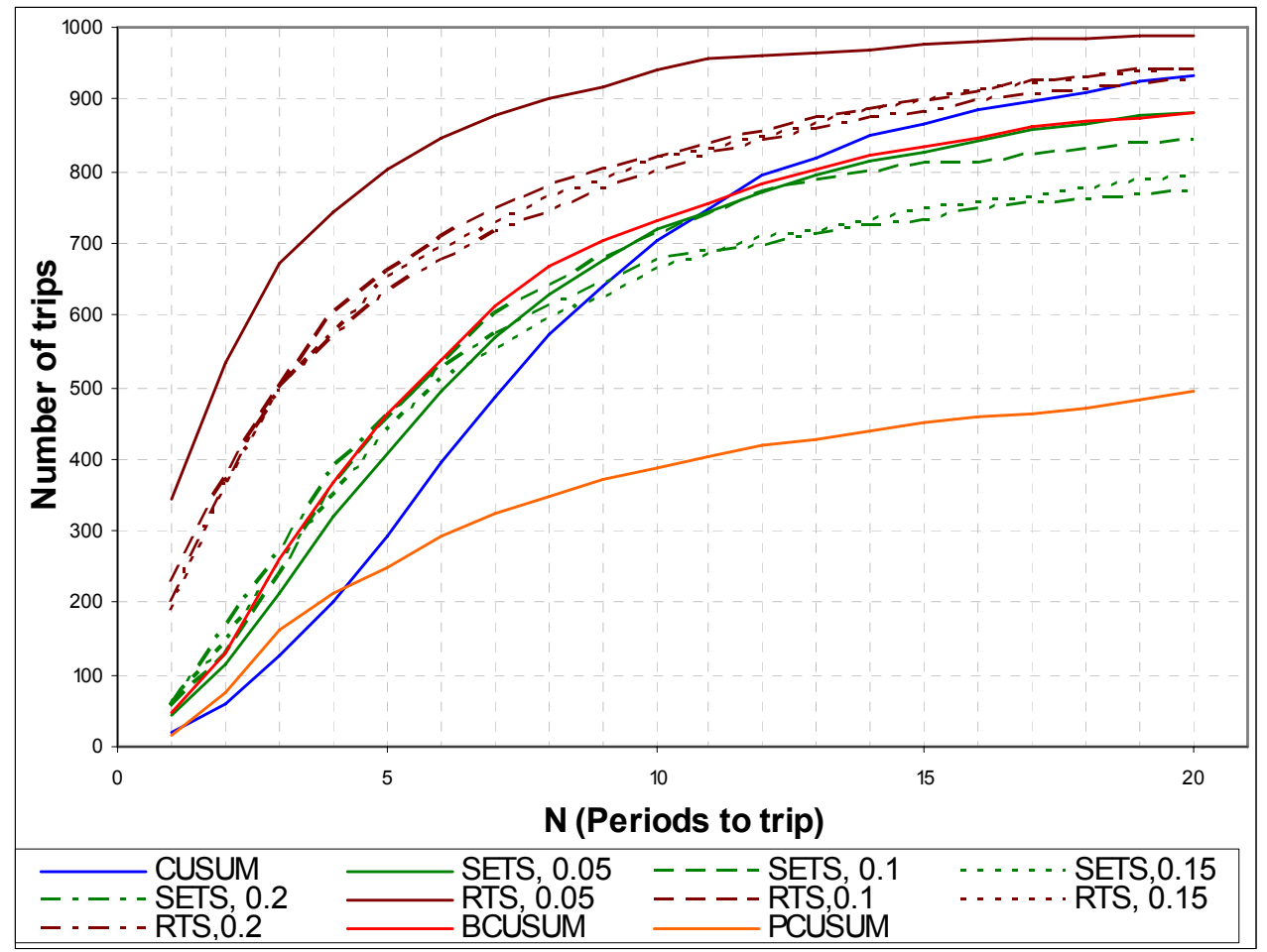

Figure 4.10 Response within $\mathrm{N}$ periods, $\mathrm{ARL}=50, R_{a}=0.2,1 \sigma$ step

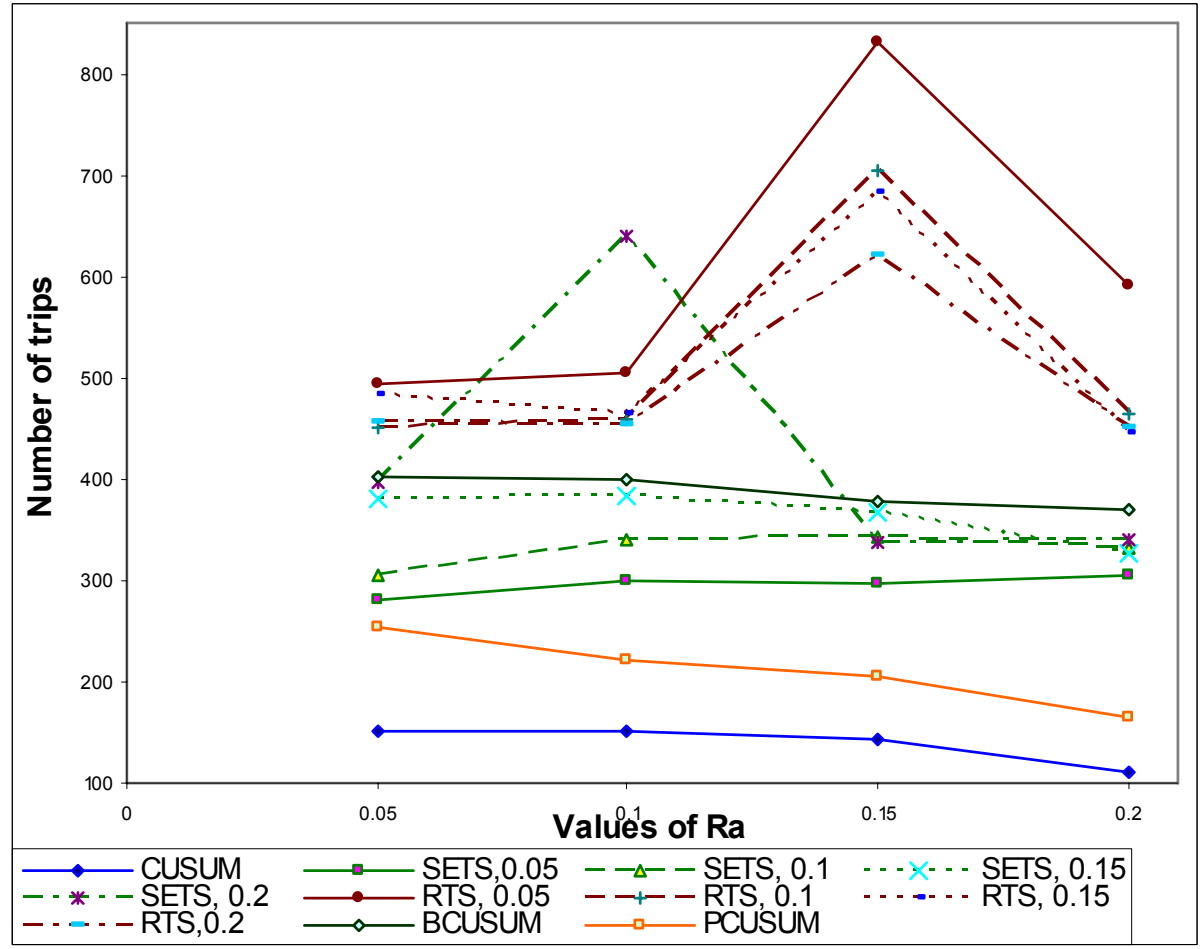

Figure 4.11 Fifth Period Response to a $1 \sigma \operatorname{step}(A R L=100)$ 
Figure 4.11 presents the number of trips that occurred within 5 periods of the actual step in demand for each tracking signal over a range of values of $R_{a}$, the average run length $(A R L 1)$ is held constant at 100. This shows very clearly that all of the autocorrelation methods dominate all the other tracking signals, one exception being smoothed error tracking signal at $R_{a}=0.1, R_{e}=0.2$. For all the autocorrelation tracking signals at $R_{a}=0.15$, and for smoothed error tracking signal at $R_{a}=0.1$ it can be noted that there is a huge spike in the number of detections. The backward CUSUM tracking signal is second best except for smoothed error tracking signal at $R_{a}=0.1$. The behavior of CUSUM and PCUSUM tracking signals remains similar to what it was when ARL is held constant at 25 and 50 .

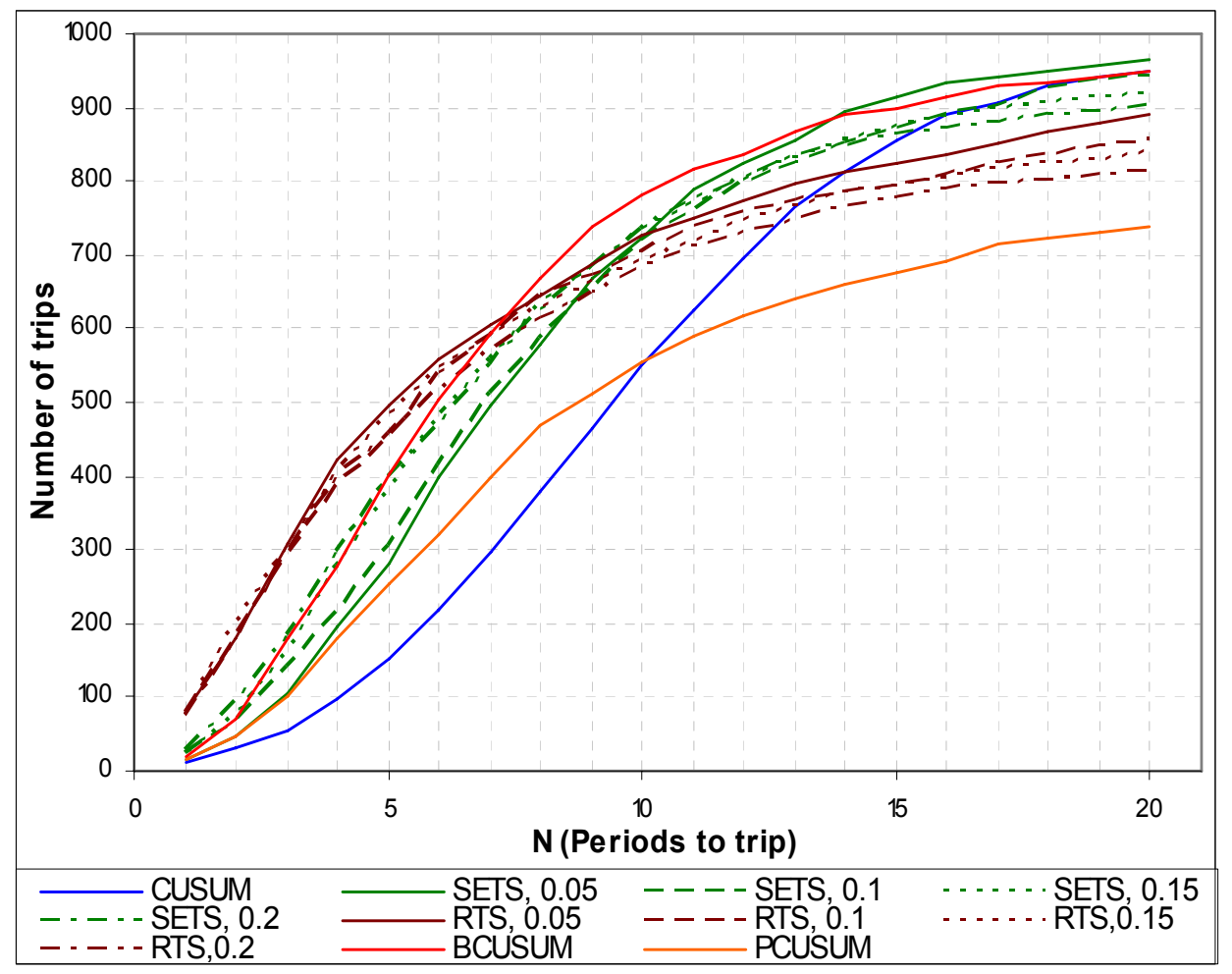

Figure 4.12 Response within $\mathrm{N}$ periods, $A R L=100, R_{a}=0.05,1 \sigma$ step 
Figure 4.12 shows response within $N$ periods for $A R L=100,1$ sigma step and for $R_{a}$ value of 0.05 . The autocorrelation tracking signals dominate for first 6 periods after which BCUSUM, smoothed error and CUSUM tracking signals take over. The detection probability for PCUSUM is higher then the CUSUM for first 9 periods after which the detection probability for PCUSUM is the lowest of all the tracking signals.

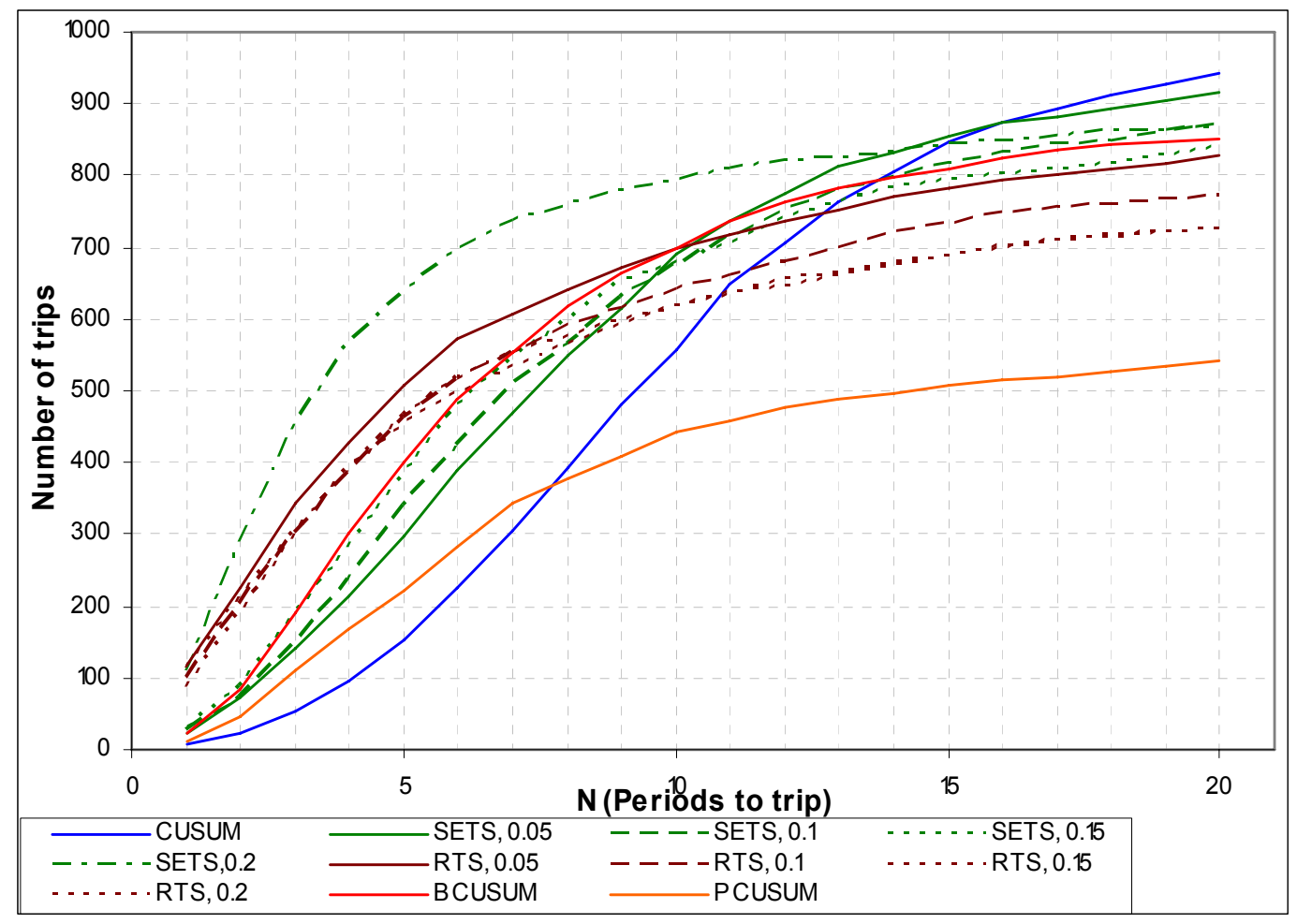

Figure 4.13 Response within $N$ periods, $A R L=100, R_{a}=0.1,1 \sigma$ step

Figure 4.13 shows response within $N$ periods for $A R L=100,1$ sigma step and for $R_{a}$ value of 0.1 . The smoothed error tracking signal with $R_{e}=0.2$ dominates all the other tracking signals until 14 periods. The CUSUM tracking signal starts the lowest and eventually overtakes all the other tracking signals. Therefore, CUSUM is the dominant choice if the goal is detection of a change, regardless of how long it takes. Figure 4.14 presents response of all the tracking signals within $N$ periods for $A R L=100,1$ sigma step 
and for $R_{a}$ value of 0.15 . The results are similar to results obtained when ARL is held constant at 25 and 50.

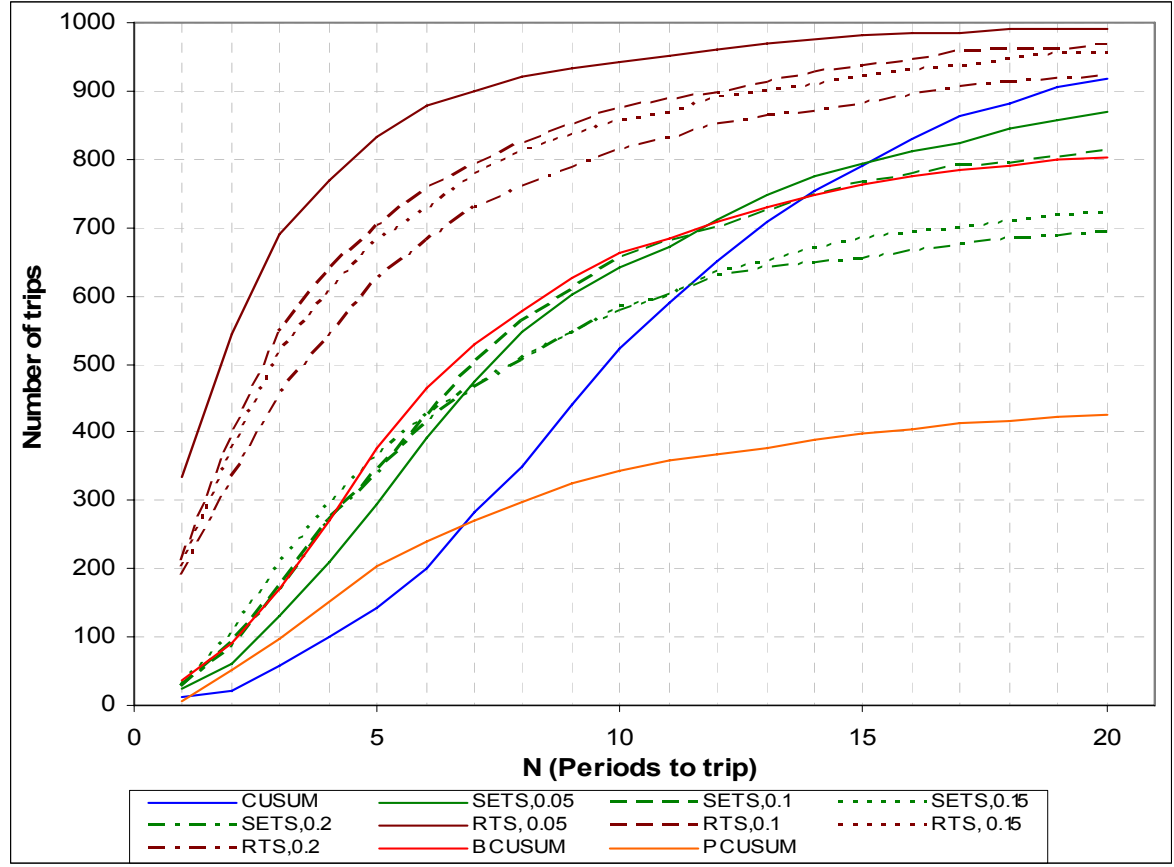

Figure 4.14 Response within $N$ periods, $A R L=100, R_{a}=0.15,1 \sigma$ step

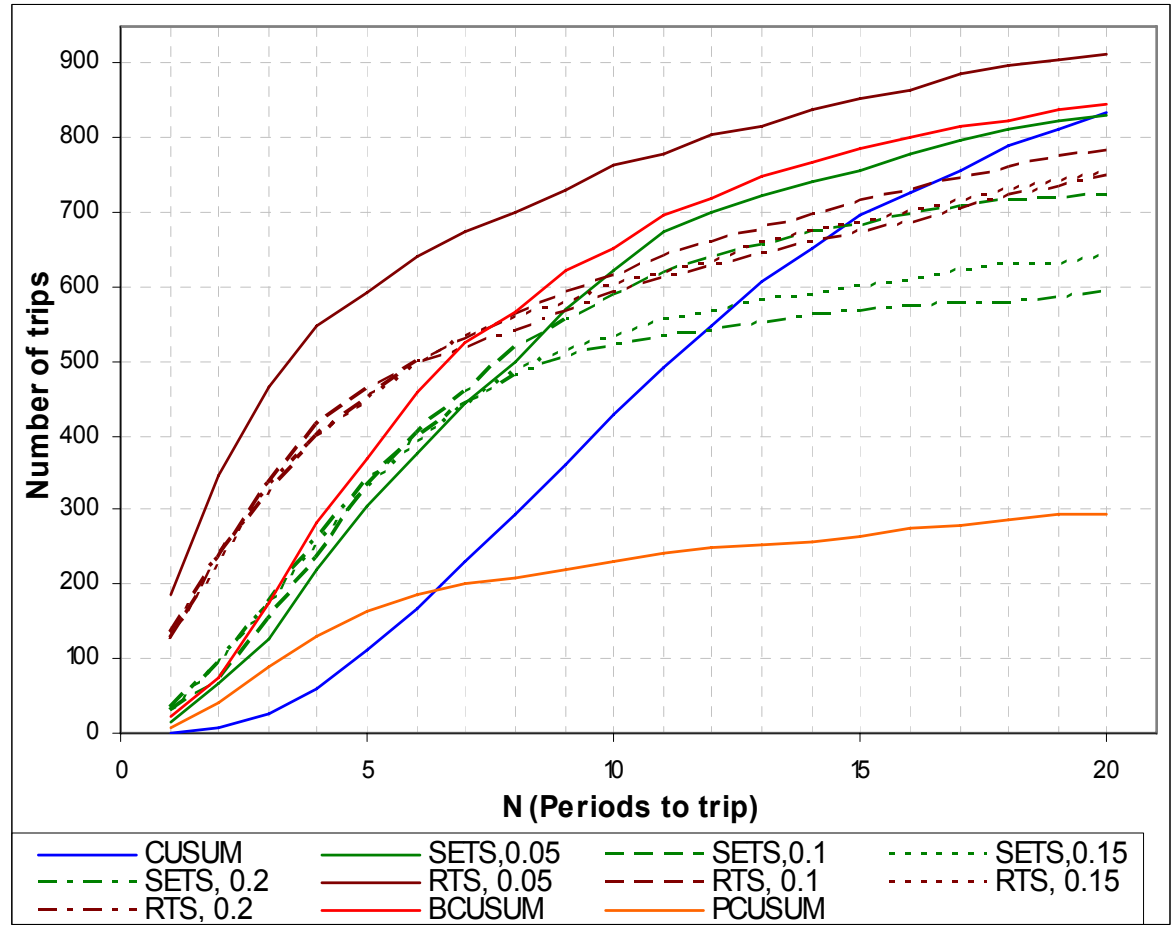

Figure 4.15 Response within $\mathrm{N}$ periods, $A R L=100, R_{a}=0.2,1 \sigma$ step 
Figure 4.15 shows response within $N$ periods for $A R L=100,1 \sigma$ step and for $R_{a}$ value of 0.1 . It can be seen that the autocorrelation tracking signal with smoothing constant, $R_{c}=0.05$ dominates all the other tracking signals. The results are similar to results obtained when ARL is held constant at 25 and 50.

The experiment was repeated for a $2 \sigma, 3 \sigma$ and a random step increase. The results obtained were similar to the response for $1 \sigma$ step. 


\section{Chapter 5}

\section{Conclusion and Future Work}

\subsection{Summary and Conclusions}

All tracking signals were compared at control limits yielding ARLs of 25, 50 and 100 periods on unbiased errors from simple exponential smoothing. Comparisons were made using smoothed values of variance (MAD, MSE, or $\sigma^{2}$ ) of forecast errors. The tracking signals were compared at step responses of $1 \sigma, 2 \sigma$, and $3 \sigma$ and also for a random step increase. The tracking signals were compared under two different categories, as follows:

\section{When all smoothing constants are equal.}

The autocorrelation tracking signal dominates all the other tracking signals when all the smoothing constants were set at $0.05, \mathrm{ARL}=25,50$ or 100 and for step responses of random or 1, 2, 3 sigma. Backward CUSUM tracking signals performed better than the other three tracking signals. When all the smoothing constants were set at 0.1 , the autocorrelation tracking signal is the best choice for unbiased ARL $=25$ where as Backward CUSUM is the best choice for unbiased ARL $=50$ or 100 . The Backward CUSUM tracking signal performed better than the other three tracking signals for unbiased $\mathrm{ARL}=25$, where as autocorrelation tracking signal and smoothed error tracking signals performed better than the other three for unbiased ARL of 50 and 100, respectively. The experiments with all smoothing constants $=0.15$ and 0.2 yielded results similar to when all the smoothing constants were set at 0.1 . 


\section{When $\boldsymbol{R}_{m}=R_{m s}=\mathbf{0 . 0 5}$ and all other smoothing constants varied from 0.05 to}

\section{2 .}

At $R_{a}=0.05,0.15$ and 0.2 the autocorrelation tracking signal with $R_{c}=0.05$ is the best choice for unbiased ARL of 25,50 or 100 . However, at $R_{a}=0.1$ the Backward CUSUM tracking signal is the best choice for unbiased ARL of 100 and the autocorrelation tracking signal with $R_{c}=0.05$ is the best choice for unbiased ARL of 25 or 50 .

The choice of $R_{m} / R_{m s}$ was based on the analysis explained in Chapter 4.

Average run length by itself is not appropriate as a measure of response to a step in the average level of demand. If a step in demand has occurred recently, the user will take immediate action to minimize the effects of the erroneous forecasts that will have occurred since the change. However, if the run length is too long, the opportunity to overcome past errors is lost; the relevant actions would be too far in the past and the current forecast will have already adjusted to the change in demand. An alternative criterion is to specify a time cutoff and a corresponding probability. The criterion used in this research is percent of trips detected within $\mathrm{N}$ periods holding constant the average run length between false alarms (ARL1). All tracking signals used $R_{m} / R_{m s}=0.05$ since we already determined that all tracking signals work best at $R_{m} / R_{m s}=0.05$. The autocorrelation tracking signal is still the best choice based on this criterion. However the autocorrelation tracking signal with different covariance smoothing parameters were better in some instances. 


\subsection{Recommendations for Future Research}

As mentioned earlier in this research the tracking signals CUSUM, Smoothed error tracking signal, Backward CUSUM tracking signal, Autocorrelation tracking signal and Parabolic CUSUM tracking signal were compared according to ARL1 criterion and also the percent of trips detected within $\mathrm{N}$ periods holding constant the average run length between false alarms (ARL1). A computer model may be developed which will incorporate both ARL1 and percent of trips detected and gives the optimal tracking signal based on both criteria. This research can be extended to compare the performance of tracking signals at unbiased ARL1 of 200, 300, 400 and 500. Also another area for further research would be to do an investigation and find out if any improvements would help to enhance the performance of the tracking signals. 


\section{Bibliography}

1. Adams, B. M., Lin, W.S. (1996). Combined control charts for forecast based monitoring schemes. Journal of Quality Technology, 28: 3, 289-301.

2. Adams, B. M., Tseng, I. (1998). Robustness of forecast based monitoring schemes. Journal of Quality Technology, 30: 4, 328-339.

3. Armstrong, J. S. (2001). Principles of Forecasting: A Handbook for Researchers and Practitioners, Kluwer Academic Publishers, Norwell, MA

4. Armstrong, J. S. (1985). Long range forecasting, John Wiley and sons.

5. Atienza, O.O., Tang, L.C., Ang, B.W. (2000). A uniformly most powerful cumulative sum scheme based on symmetry. The Statistician, 49:part2, 209-217.

6. Batty, M., (1969). Monitoring an exponential smoothing forecasting system. Operational Research Quarterly, 20: 319-325.

7. Box, G. and Jenkins, G. (1976). Time Series Analysis, Forecasting and Control, Revised Edition, Holden-Day, San Francisco, CA.

8. Brown, R. G., (1959). Statistical Forecasting for Inventory Control. McGraw-Hill, New York.

9. Brown, R. G., (1963). "Smoothing, Forecasting and Prediction of Discrete Time Series", Prentice-Hall, Englewood Cliffs, N.J.

10. Brown, R. G., (1971). Detection of turning points in a time series. Decision sciences, 2: 383-403.

11. Brown, R. G., (1982). Advanced service parts inventory control, Norwich, VT: Materials Management Systems, Inc., Chapters 3 and 9. 
12. Bunn, D. W. (1981), 'Adaptive forecasting using the Kalman filter', Omega, 9: 323-324.

13. Chow, W. M., (1965). Adaptive control of the exponential smoothing constant, Journal of Industrial Engineering, 16: 314-317.

14. Coutie, G. et al., (1964). Short-Term Forecasting, ICT Monograph No. 2, Edinburgh; Oliver and Boyd.

15. Deng Y, Jaraiedi M, 1skander W. (2003) Tracking Signal Test to Monitor an Intelligent Time Series Forecasting Model, Intelligent Manufacturing, 29-30: 149160.

16. Enns, P. G. et al., (1982) 'Forecasting applications of an adaptive multiple exponential smoothing model', Management Science, 28: 1035-1044.

17. Fildes, R., (1979) 'Quantitative forecasting - the state of the art: extrapolative methods', Journal of the Operational Research Society, 30: 691 - 710.

18. Flowers, A. D., (1980) 'A simulation study of smoothing constant limits for an adaptive forecasting system', Journal of Operations Management, 2: 85-94.

19. Gardner, E. S., (1983). Automatic monitoring of forecast errors. Journal of Forecasting, 2(1): 1-22.

20. Gardner, E. S., (1985). CUSUM vs. smoothed error forecast monitoring schemes: Some simulation results, Journal of the Operational Research Society 36: 43-47.

21. Gardner, E. S., (1985a). Exponential smoothing: The state of the art, Journal of Forecasting, 4: 1-28.

22. Gilchrist, W. G., (1967). Methods of estimation using discounting, Journal of the Royal Statistical Society (B), 29: 355-369. 
23. Gilchrist, W. G., (1976). Statistical Forecasting, London: Wiley.

24. Golder, E.R. and Settle, J.G., (1976). Monitoring schemes in short-term forecasting, Operational research quarterly, 27: 489-501.

25. Harrison, P. J. and Davies, L. O. (1964). The use of cumulative sum (CUSUM) techniques for the control of routine forecasts of product demand. Operations Research, 12: 325-333.

26. Makridakis S., Wheelwright S., and McGee V (1983). Forecasting: Methods and Applications. John Wiley and Sons, New York.

27. Makridakis S., and Wheelwright S. (1989). Forecasting Methods for Management. John Wiley \& Sons, Inc.

28. Martino, J. (1983). Technological forecasting for decision making. Elsevier Science Publishing Company, New York.

29. McClain, J.O., (1988). Dominant tracking signals. International Journal of Forecasting. 4: 563-572.

30. Montgomery, D. C., (1970). Adaptive control of exponential smoothing parameters by evolutionary operation, AIIE Transactions, 2: 268-269.

31. Montgomery, D. C., Johnson, L. A. and Gardiner, J. S. (1990). Forecasting \& Time Series Analysis. McGraw-Hill, Inc., 2nd edition.

32. Nelson, C. R.(1973). Applied time series analysis for managerial forecasting, Holden Day, Inc., San Francisco.

33. Nembhard, H.B., Kao, M.S. (2003), Adaptive forecast based monitoring for dynamic systems, Technometrics, 45: 3, 208-219. 
34. O’Donovan, T. M., (1983). Short term Forecasting, John Wiley and sons, New York.

35. Rao, A. G. and Shapiro, A., (1970) 'Adaptive smoothing using evolutionary spectra', Management Science, 17: 208218.

36. Roberts, S.D. and Reed R., (1969). The development of a self adaptive forecasting technique. AIIE transactions 1: 314-322.

37. Shim, J.K., Siegel, J.G., Liew, C.J. (1994), Strategic Business Forecasting, St Lucie Press, New York.

38. Shone, M. L. (1967), 'Viewpoints', Operational Research Quarterly, 18: 318-319.

39. Stig, J.W. (1997), Parabolic CUSUM control charts. Communications in statistics - Simulation and computation, 26: 107-123.

40. Trig, W., (1964). Monitoring a forecasting system. Operations Research Quarterly, 15: 271-274.

41. Trigg, D. W. and Leach, A. G., (1967) "Exponential smoothing with an adaptive response rate', Operational Research Quarterly, 18: 53-59.

42. Wald, A., (1947) Sequential Analysis. John Wiley \& Sons, Inc.

43. Whybark, D. C, (1973) 'A comparison of adaptive forecasting techniques', Logistics Transportation Review, 8: 13-36. 